\title{
Synthesis of Benzothienobenzofurans via Annulation of Electrophilic Benzothiophenes with Phenols
}

\author{
Akhil Krishnan R., ${ }^{a}$ Sheba Ann Babu, ${ }^{a, b}$ Nitha P. R., ${ }^{a, b}$ Jagadeesh Krishnan ${ }^{a}$ and Jubi Johna,b* \\ ${ }^{a}$ Chemical Sciences and Technology Division, CSIR-National Institute for Interdisciplinary Science and Technology \\ (CSIR-NIIST), Thiruvananthapuram 695019, India. \\ ${ }^{\mathrm{b}}$ Academy of Scientific and Innovative Research (AcSIR), Ghaziabad-201002, India. \\ *E-mail: jubijohn@niist.res.in
}

\section{Table of Contents}

\begin{tabular}{|c|c|c|}
\hline 1. & General Methods & :S2 \\
\hline 2. & Procedure for the synthesis of 3-nitrobenzothiophene and $\mathrm{N}$-Ts-3-nitro-indole & : S2-S3 \\
\hline 3. & $\begin{array}{l}\text { Experimental procedure for the reaction between 3-nitrobenzothiophene and } \\
\text { phenols }\end{array}$ & :S3 \\
\hline 4. & Synthesis and characterization of benzothieno[3,2-b] benzofurans & : S3-S18 \\
\hline 5. & Procedure for scale up of $\mathbf{3 a}$ & : S18 \\
\hline 6. & $\begin{array}{l}\text { Procedure for the synthesis of benzofuro[3,2-b]indole by the annulation of } \\
\text { electrophilic indole }\end{array}$ & : S19 \\
\hline 7. & References & : S20 \\
\hline 8. & ${ }^{1} \mathrm{H}$ NMR $\&{ }^{13} \mathrm{C}$ NMR Spectra & : S21-S54 \\
\hline 9. & Single crystal X-ray of $\mathbf{3 a}$ & : S55-S56 \\
\hline
\end{tabular}




\section{General methods}

All chemicals were of the best grade commercially available and were used without further purification. All solvents were purified according to the standard procedures; dry solvents were obtained according to the literature methods and stored over molecular sieves. Analytical thinlayer chromatography was performed on polyester sheets pre-coated with silica gel containing fluorescent indicator (POLYGRAMSIL G/UV254). Gravity column chromatography was performed using neutral alumina, and mixtures of ethyl acetate hexanes were used for elution. Melting points were determined using a calibrated digital melting point apparatus (Büchi 530 melting point apparatus). IR spectra were recorded on Bruker FT-IR spectrometer. NMR spectra were recorded with Bruker Avance-300 (300 MHz for ${ }^{1} \mathrm{H} N M R, 75 \mathrm{MHz}$ for $\left.{ }^{13} \mathrm{C}\left\{{ }^{1} \mathrm{H}\right\} \mathrm{NMR}\right)$ and Bruker AMX-500 (500 MHz for ${ }^{1} \mathrm{H}$ NMR, $125 \mathrm{MHz}$ for ${ }^{13} \mathrm{C}\left\{{ }^{1} \mathrm{H}\right\} \mathrm{NMR}$ ) instruments. All spectra were measured at $300 \mathrm{~K}$, unless otherwise specified. The chemical shifts $\delta$ are given in ppm and referenced to the external standard TMS or internal solvent standard. ${ }^{1} \mathrm{H}$ NMR coupling constants $(\mathrm{J})$ are reported in Hertz $(\mathrm{Hz})$ and multiplicities are indicated as follows $\mathrm{s}$ (singlet), $\mathrm{d}$ (doublet), $\mathrm{t}$ (triplet), q (quartet), m (multiplet), dd (doublet of doublets). Mass spectra were performed with a ThermoFinnigan MAT95XL, a ThermoFisher Scientific LTQ Orbitrap Velos, and an Agilent 6890 gas chromatograph with JMS-T100GC spectrometer or with a ESI/ HRMS at 60,000 resolution using ThermoScientific Exactive mass spectrometer with orbitrap analyzer. Gas chromatographic analysis was performed using GCMS-TQ8030 SHIMADZU.

\section{Procedure for the synthesis of 3-nitrobenzothiophene and $\mathbf{N}$-Ts-3-nitro-indole}

- 3-nitrobenzothiophene ${ }^{1}$, 2-nitrobenzothiophene ${ }^{2}$, 5-bromo-3-nitrobenzothiophene ${ }^{1}$, 5cyano-3-nitrobenzothiophene $\mathrm{e}^{1}$ and $\mathrm{N}$-Ts-3-nitro-indole ${ }^{1}$ were prepared according to previous literature. 


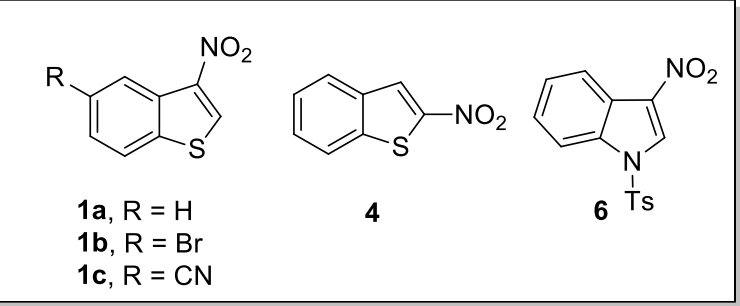

3. Experimental procedure for the reaction between 3-nitrobenzothiophene and phenols: $A$ mixture of 3-nitrobenzothiophene ( $100 \mathrm{mg}, 1.0$ equiv.), phenol (1.3 equiv.) and $\mathrm{K}_{2} \mathrm{CO}_{3}$ ( 0.5 equiv.) was weighed into a dry reaction tube. Distilled EtOH $(2.0 \mathrm{ml})$ was added and allowed to stir at 80 ${ }^{\circ} \mathrm{C}$ on a reaction block for 24 hours. After completion of the reaction as indicated from the TLC, the solvent was removed under vacuum. The residue was then purified by column chromatography (silica gel, eluent: hexane) to afford the corresponding products.

\section{Synthesis and characterization of benzothieno[3,2-b]benzofurans}

\section{8-methoxybenzothieno[3,2-b]benzofuran (3a)}<smiles>COc1ccc2oc3c4ccccc4sc3c2c1</smiles>

The reaction was performed according to the general procedure with 3-nitrobenzothiophene 1a (100 mg, $0.56 \mathrm{mmol})$, 4-methoxy phenol $2 \mathrm{a}\left(91 \mathrm{mg}, 0.73 \mathrm{mmol}\right.$ ) and $\mathrm{K}_{2} \mathrm{CO}_{3}(39 \mathrm{mg}, 0.28 \mathrm{mmol})$ at $80{ }^{\circ} \mathrm{C}$ for $24 \mathrm{~h}$. After solvent removal, the residue was purified by silica gel column chromatography (hexane) to afford the desired product 3a as a colourless solid (133 $\mathrm{mg}, 94 \%$ ). The compound was crystallized by slow evaporation method from a 1:1 mixture of toluene and diethylether.

Analytical data of 3a: Mp: 98-100 ${ }^{\circ} \mathrm{C}$. IR (neat) $v_{\max }: 2919,1454,1256,834,746 \mathrm{~cm}^{-1} .{ }^{1} \mathrm{H}$ NMR $(500$ $\left.\mathrm{MHz}, \mathrm{CDCl}_{3}, \mathrm{TMS}\right): \delta 7.89(\mathrm{~d}, J=8.0 \mathrm{~Hz}, 1 \mathrm{H}), 7.78(\mathrm{~d}, J=8.0 \mathrm{~Hz}, 1 \mathrm{H}), 7.44(\mathrm{~d}, J=9.0 \mathrm{~Hz}, 1 \mathrm{H}), 7.37$ (t, J= $7.5 \mathrm{~Hz}, 1 \mathrm{H}), 7.29(\mathrm{t}, J=7.5 \mathrm{~Hz}, 1 \mathrm{H}), 7.08(\mathrm{~s}, 1 \mathrm{H}), 6.88(\mathrm{~d}, J=9.0 \mathrm{~Hz}, 1 \mathrm{H}), 3.81(\mathrm{~s}, 3 \mathrm{H}) \mathrm{ppm}$. ${ }^{13} \mathrm{C}\left\{{ }^{1} \mathrm{H}\right\}$ NMR $\left(125 \mathrm{MHz}, \mathrm{CDCl}_{3}\right): \delta 156.2,153.8,153.6,142.0,125.2,125.0,124.9,124.5,124.4$, 
119.7, 118.5, 113.3, 113.0, 102.4, 56.0 ppm. HRMS (ESI-Orbitrap) m/z: $(\mathrm{M}+\mathrm{H})^{+}$calcd for $\mathrm{C}_{15} \mathrm{H}_{11} \mathrm{O}_{2} \mathrm{~S}$ 255.0474 , found 255.0477.

\section{Benzothieno[3,2-b]benzofuran $(3 b)^{3}$}

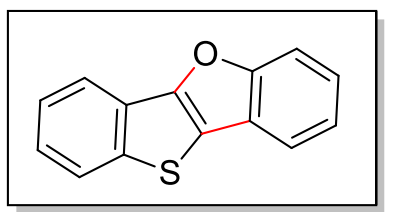

The reaction was performed according to the general procedure with 3-nitrobenzothiophene 1a (100 mg, $0.56 \mathrm{mmol})$, phenol $2 \mathbf{b}(69 \mathrm{mg}, 0.73 \mathrm{mmol})$ and $\mathrm{K}_{2} \mathrm{CO}_{3}\left(39 \mathrm{mg}, 0.28 \mathrm{mmol}\right.$ ) at $80{ }^{\circ} \mathrm{C}$ for $24 \mathrm{~h}$. After solvent removal, the residue was purified by silica gel column chromatography (hexane) to afford the desired product $\mathbf{3 b}$ as a colourless solid ( $84 \mathrm{mg}, 67 \%$ ).

Analytical data of 3b: Mp: $120-122{ }^{\circ} \mathrm{C}$. IR (neat) $v_{\max }: 2919,1582,1182,886,729 \mathrm{~cm}^{-1} .{ }^{1} \mathrm{H}$ NMR (500 MHz, CDCl $3, \mathrm{TMS}$ ): $\delta 7.93$ (d, $J=8.0 \mathrm{~Hz}, 1 \mathrm{H}), 7.81$ (d, $J=8.0 \mathrm{~Hz}, 1 \mathrm{H}), 7.65(\mathrm{~d}, J=7.5 \mathrm{~Hz}, 1 \mathrm{H})$, $7.58(\mathrm{~d}, J=8.0 \mathrm{~Hz}, 1 \mathrm{H}), 7.40(\mathrm{t}, J=7.5 \mathrm{~Hz}, 1 \mathrm{H}), 7.33-7.26(\mathrm{~m}, 3 \mathrm{H}) \mathrm{ppm} .{ }^{13} \mathrm{C}\left\{{ }^{1} \mathrm{H}\right\} \mathrm{NMR}(125 \mathrm{MHz}$, $\left.\mathrm{CDCl}_{3}\right): \delta 158.8,153.0,142.0,125.2,125.0,124.9,124.9,124.4,124.1,123.3,119.7,119.6,118.6$, $112.6 \mathrm{ppm}$.

\section{8-methylbenzothieno[3,2-b]benzofuran $(3 c)^{3}$}

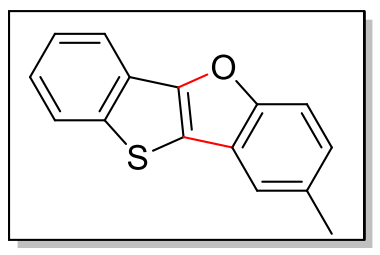

The reaction was performed according to the general procedure with 3-nitrobenzothiophene 1a (100 mg, $0.56 \mathrm{mmol}), p$ - cresol $2 \mathrm{c}(79 \mathrm{mg}, 0.73 \mathrm{mmol})$ and $\mathrm{K}_{2} \mathrm{CO}_{3}(39 \mathrm{mg}, 0.28 \mathrm{mmol})$ at $80{ }^{\circ} \mathrm{C}$ for $24 \mathrm{~h}$. After solvent removal, the residue was purified by silica gel column chromatography (hexane) to afford the desired product $3 \mathrm{c}$ as a colourless solid (100 mg, $75 \%$ ).

Analytical data of 3c: Mp: $96-98^{\circ} \mathrm{C}$. IR (neat) $v_{\max }: 3066,1389,1260,787,745,725 \mathrm{~cm}^{-1} .{ }^{1} \mathrm{H}$ NMR $\left(500 \mathrm{MHz}^{\mathrm{CDCl}} \mathrm{CD}_{3}: \delta 7.91(\mathrm{~d}, J=8.0 \mathrm{~Hz}, 1 \mathrm{H}), 7.79(\mathrm{~d}, J=8.5 \mathrm{~Hz}, 1 \mathrm{H}), 7.44-7.43(\mathrm{~m}, 2 \mathrm{H}), 7.39(\mathrm{t}, J=\right.$ 
$7.0 \mathrm{~Hz}, 1 \mathrm{H}), 7.30(\mathrm{t}, J=7.5 \mathrm{~Hz}, 1 \mathrm{H}), 7.11(\mathrm{~d}, J=8.5 \mathrm{~Hz}, 1 \mathrm{H}), 2.42(\mathrm{~s}, 3 \mathrm{H}) \mathrm{ppm} .{ }^{13} \mathrm{C}\left\{{ }^{1} \mathrm{H}\right\} \mathrm{NMR}(125$ $\left.\mathrm{MHz}, \mathrm{CDCl}_{3}\right): \delta 157.2,153.2,142.0,132.9,126.1,125.3,124.9,124.8,124.4,124.1,119.6,119.5$, $118.4,112.0,21.4 \mathrm{ppm}$.

\section{8-(tert-butyl)benzothieno[3,2-b]benzofuran $(3 d)^{3}$}<smiles>CC(C)(C)c1ccc2oc3c4ccccc4sc3c2c1</smiles>

The reaction was performed according to the general procedure with 3-nitrobenzothiophene 1a (100 mg, $0.56 \mathrm{mmol}$ ), 4-tert-butylphenol $2 \mathrm{~d}\left(110 \mathrm{mg}, 0.73 \mathrm{mmol}\right.$ ) and $\mathrm{K}_{2} \mathrm{CO}_{3}(39 \mathrm{mg}, 0.28 \mathrm{mmol})$ at $80{ }^{\circ} \mathrm{C}$ for $24 \mathrm{~h}$. After solvent removal, the residue was purified by silica gel column chromatography (hexane) to afford the desired product $\mathbf{3 d}$ as a colourless solid (127 $\mathrm{mg}, 81 \%$ ).

Analytical data of $3 d$ : Mp: $116-118^{\circ} \mathrm{C}$. IR (neat) $v_{\max }: 2940,2363,1596,1453,1200,809,716 \mathrm{~cm}^{-}$ 1. ${ }^{1} \mathrm{H}$ NMR (500 MHz, CDCl 3 ): $\delta 7.90(\mathrm{~d}, J=8.0 \mathrm{~Hz}, 1 \mathrm{H}), 7.78(\mathrm{~d}, J=8.0 \mathrm{~Hz}, 1 \mathrm{H}), 7.64(\mathrm{~s}, 1 \mathrm{H}), 7.47$ $(\mathrm{d}, J=8.5 \mathrm{~Hz}, 1 \mathrm{H}), 7.38-7.34(\mathrm{~m}, 2 \mathrm{H}), 7.28(\mathrm{t}, J=7.5 \mathrm{~Hz}, 1 \mathrm{H}), 1.34(\mathrm{~s}, 9 \mathrm{H}) \mathrm{ppm} .{ }^{13} \mathrm{C}\left\{{ }^{1} \mathrm{H}\right\} \mathrm{NMR}(125$ $\mathrm{MHz}_{\mathrm{CDCl}}$ ): $\delta 157.0,153.2,146.6,141.9,125.3,124.9,124.7,124.4,123.7,122.8,119.6,118.8$, 116.0, 111.8, 34.9, 31.9 ppm.

\section{8-phenylbenzothieno[3,2-b]benzofuran $(3 e)^{3}$}

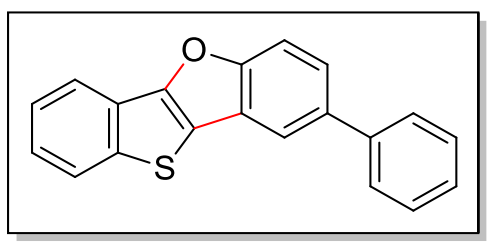

The reaction was performed according to the general procedure with 3-nitrobenzothiophene 1a (100 mg, $0.56 \mathrm{mmol})$, 4- phenylphenol 2e (124 mg, $0.73 \mathrm{mmol})$ and $\mathrm{K}_{2} \mathrm{CO}_{3}(39 \mathrm{mg}, 0.28 \mathrm{mmol})$ at $80{ }^{\circ} \mathrm{C}$ for $24 \mathrm{~h}$. After solvent removal, the residue was purified by silica gel column chromatography (hexane) to afford the desired product $3 e$ as a colourless solid (122 mg, 73 \%). 
Analytical data of $3 e: M p: 138-140{ }^{\circ} \mathrm{C}$. IR (neat) $v_{\max }$ : 3055, 1442, 1260, 868, $682 \mathrm{~cm}^{-1}$. ${ }^{1} \mathrm{H}$ NMR $\left(500 \mathrm{MHz}, \mathrm{CDCl}_{3}\right): \delta 7.93(\mathrm{~d}, J=7.5 \mathrm{~Hz}, 1 \mathrm{H}), 7.83-7.79(\mathrm{~m}, 2 \mathrm{H}), 7.61-7.57(\mathrm{~m}, 3 \mathrm{H}), 7.51(\mathrm{~d}, J=8.5$ $\mathrm{Hz}, 1 \mathrm{H}), 7.41-7.37(\mathrm{~m}, 3 \mathrm{H}), 7.33-7.27(\mathrm{~m}, 2 \mathrm{H}) \mathrm{ppm} .{ }^{13} \mathrm{C}\left\{{ }^{1} \mathrm{H}\right\} \mathrm{NMR}\left(125 \mathrm{MHz}, \mathrm{CDCl}_{3}\right): \delta 158.4$, $153.6,142.1$, 141.2, 137.1, 128.8, 127.5, 127.2, 125.2, 125.0, 124.6, 124.5, 124.4, 119.8, 118.1, $112.7 \mathrm{ppm}$.

\section{8-bromobenzothieno[3,2-b]benzofuran $(3 g)^{4}$}<smiles>Brc1ccc2c(c1)C=CC=C1C=c3c(sc4ccccc34)=C1O2</smiles>

The reaction was performed according to the general procedure with 3-nitrobenzothiophene 1a (100 mg, $0.56 \mathrm{mmol}$ ), 4- bromophenol $2 \mathrm{~g}$ (126 mg, $0.73 \mathrm{mmol}$ ) and $\mathrm{K}_{2} \mathrm{CO}_{3}(39 \mathrm{mg}, 0.28 \mathrm{mmol}$ ) at $80{ }^{\circ} \mathrm{C}$ for $24 \mathrm{~h}$. After solvent removal, the residue was purified by silica gel column chromatography (hexane) to afford the desired product $3 \mathrm{~g}$ as a colourless solid (122 $\mathrm{mg}, 72 \%$ ).

Analytical data of $3 \mathrm{~g}$ : Mp: $162-164{ }^{\circ} \mathrm{C}$. IR (neat) $v_{\max }$ : 3040, 1432, 1257, 857, $746 \mathrm{~cm}^{-1} .{ }^{1} \mathrm{H}$ NMR $\left(500 \mathrm{MHz}, \mathrm{CDCl}_{3}\right): \delta 7.91(\mathrm{~d}, J=7.5 \mathrm{~Hz}, 1 \mathrm{H}), 7.80-7.77(\mathrm{~m}, 2 \mathrm{H}), 7.43-7.37(\mathrm{~m}, 3 \mathrm{H}), 7.34-7.31(\mathrm{~m}$, 1H) ppm. ${ }^{13} \mathrm{C}\left\{{ }^{1} \mathrm{H}\right\} \mathrm{NMR}\left(125 \mathrm{MHz}, \mathrm{CDCl}_{3}\right): \delta 157.5,154.0,142.3,127.7,125.9,125.5,125.1,124.8$, $124.4,122.3,119.9,117.6,116.4,113.9$ ppm.

\section{8-chlorobenzothieno[3,2-b]benzofuran $(3 \mathrm{~h})^{3}$}<smiles></smiles>

The reaction was performed according to the general procedure with 3-nitrobenzothiophene 1a (100 mg, $0.56 \mathrm{mmol}$ ), 4-chlorophenol $2 \mathrm{~h}$ (94 mg, $0.73 \mathrm{mmol}$ ) and $\mathrm{K}_{2} \mathrm{CO}_{3}(39 \mathrm{mg}, 0.28 \mathrm{mmol}$ ) at 80 ${ }^{\circ} \mathrm{C}$ for $24 \mathrm{~h}$. After solvent removal, the residue was purified by silica gel column chromatography (hexane) to afford the desired product $\mathbf{3 h}$ as a colourless solid (107 mg, $74 \%$ ). 
Analytical data of $3 \mathrm{~h}$ : Mp: $158-160{ }^{\circ} \mathrm{C}$. IR (neat) $\mathrm{v}_{\max }$ : 2919, 1429, 1062, 789, 745, $662 \mathrm{~cm}^{-1} .{ }^{1} \mathrm{H}$ $\operatorname{NMR}\left(500 \mathrm{MHz}, \mathrm{CDCl}_{3}\right): \delta 7.92(\mathrm{~d}, J=8.0 \mathrm{~Hz}, 1 \mathrm{H}), 7.80(\mathrm{~d}, J=7.5 \mathrm{~Hz}, 1 \mathrm{H}), 7.62(\mathrm{~s}, 1 \mathrm{H}), 7.48(\mathrm{~d}, J=$ $8.5 \mathrm{~Hz}, 1 \mathrm{H}), 7.41(\mathrm{t}, J=7.0 \mathrm{~Hz}, 1 \mathrm{H}), 7.33(\mathrm{t}, J=7.0 \mathrm{~Hz}, 1 \mathrm{H}), 7.25(\mathrm{~d}, J=8.5 \mathrm{~Hz}, 1 \mathrm{H}) \mathrm{ppm} .{ }^{13} \mathrm{C}\left\{{ }^{1} \mathrm{H}\right\}$ NMR (125 MHz, $\left.\mathrm{CDCl}_{3}\right): \delta$ 157.1, 154.2, 142.2, 129.0, 125.4, 125.3, 125.1, 125.0, 124.8, 124.4, $119.9,119.3,117.8,113.5$ ppm.

\section{7-methoxybenzothieno[3,2-b]benzofuran (3i)}

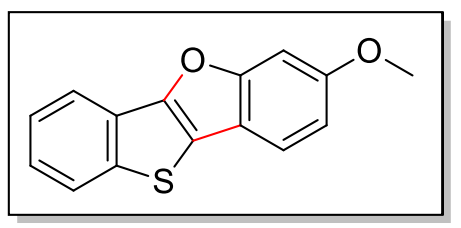

The reaction was performed according to the general procedure with 3-nitrobenzothiophene 1a (100 mg, $0.56 \mathrm{mmol}$ ), 3- methoxy phenol $2 \mathrm{i}\left(91 \mathrm{mg}, 0.73 \mathrm{mmol}\right.$ ) and $\mathrm{K}_{2} \mathrm{CO}_{3}(39 \mathrm{mg}, 0.28 \mathrm{mmol}$ ) at $80{ }^{\circ} \mathrm{C}$ for $24 \mathrm{~h}$. After solvent removal, the residue was purified by silica gel column chromatography (hexane) to afford the desired product $\mathbf{3 i}$ as a colourless solid (85 $\mathrm{mg}, 60 \%$ ).

Analytical data of 3i: Mp: $98-100{ }^{\circ} \mathrm{C}$. IR (neat) $v_{\max }$ : 3095,2914, 1491, 1148, $1107 \mathrm{~cm}^{-1} .{ }^{1} \mathrm{H}$ NMR (500 MHz, CDCl $3, \mathrm{TMS}): \delta 7.88(\mathrm{~d}, J=8.0 \mathrm{~Hz}, 1 \mathrm{H}), 7.79(\mathrm{~d}, J=8.0 \mathrm{~Hz}, 1 \mathrm{H}), 7.52(\mathrm{~d}, J=8.5 \mathrm{~Hz}, 1 \mathrm{H})$, $7.38(\mathrm{t}, J=7.0 \mathrm{~Hz}, 1 \mathrm{H}), 7.28(\mathrm{t}, J=7.5 \mathrm{~Hz}, 1 \mathrm{H}), 7.12(\mathrm{~s}, 1 \mathrm{H}), 6.90(\mathrm{~d}, J=8.5 \mathrm{~Hz}, 1 \mathrm{H}), 3.83(\mathrm{~s}, 3 \mathrm{H})$ ppm. ${ }^{13} \mathrm{C}\left\{{ }^{1} \mathrm{H}\right\} \mathrm{NMR}\left(125 \mathrm{MHz}, \mathrm{CDCl}_{3}\right): 156.2,153.8,153.6,142.0,125.2,125.0,124.9,124.5,124.4$, 119.7, 118.5, 113.3, 113.0, 102.4, 56.0 ppm. HRMS (ESI-Orbitrap) m/z: $(M+H)^{+}$calcd for $\mathrm{C}_{15} \mathrm{H}_{10} \mathrm{O}_{2} \mathrm{~S} 255.0474$, found 255.0464 .

\section{6-methoxybenzothieno[3,2-b]benzofuran (3j)}

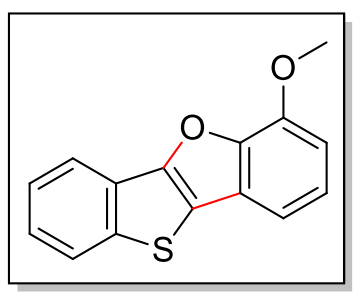

The reaction was performed according to the general procedure with 3-nitrobenzothiophene 1a (100 mg, $0.56 \mathrm{mmol}$ ), 2- methoxy phenol 2j (91 mg, $0.73 \mathrm{mmol}$ ) and $\mathrm{K}_{2} \mathrm{CO}_{3}(39 \mathrm{mg}, 0.28 \mathrm{mmol})$ at 
$80{ }^{\circ} \mathrm{C}$ for $24 \mathrm{~h}$. After solvent removal, the residue was purified by silica gel column chromatography (hexane) to afford the desired product $\mathbf{3} \mathbf{j}$ as a colourless solid (91 $\mathrm{mg}, 64 \%$ ).

Analytical data of $\mathbf{3 j}$ : Mp: $98-100{ }^{\circ} \mathrm{C}$. IR (neat) $\mathrm{V}_{\max }: 2956,1729,1262,1078,801,714 \mathrm{~cm}^{-1} .{ }^{1} \mathrm{H}$ NMR (500 MHz, CDCl $3, T M S): \delta 8.01$ (d, J = 8.0 Hz, 1H), 7.81 (d, J = 8.0 Hz, 1H), 7.40 (t, J = $7.5 \mathrm{~Hz}$, 1H), $7.32(\mathrm{t}, J=7.5 \mathrm{~Hz}, 1 \mathrm{H}), 7.27(\mathrm{~d}, J=7.5 \mathrm{~Hz}, 1 \mathrm{H}), 7.21(\mathrm{t}, J=8.0 \mathrm{~Hz} 1 \mathrm{H}), 6.87(\mathrm{~d}, J=8.0 \mathrm{~Hz}, 1 \mathrm{H})$, 4.03 (s, 3H) ppm. ${ }^{13} \mathrm{C}\left\{{ }^{1} \mathrm{H}\right\}$ NMR $\left(125 \mathrm{MHz}, \mathrm{CDCl}_{3}\right): \delta 153.0,147.8,145.9,142.1,125.5,125.2,125.0$, 124.9, 124.4, 124.0, 119.9, 119.0, 112.1, 107.4, 56.2 ppm. HRMS (ESI-Orbitrap) m/z: (M) calcd for $\mathrm{C}_{15} \mathrm{H}_{10} \mathrm{O}_{2} \mathrm{~S} 254.0396$, found 254.0395 .

\section{6-phenylbenzothieno[3,2-b]benzofuran $(3 k)$}

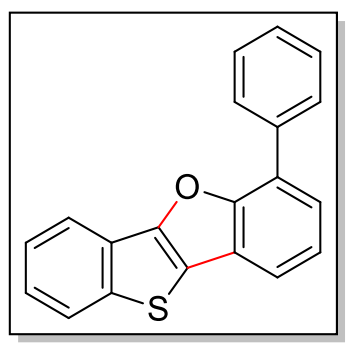

The reaction was performed according to the general procedure with 3-nitrobenzothiophene 1a (100 mg, $0.56 \mathrm{mmol}$ ), 2- phenylphenol 2k (124 mg, $0.73 \mathrm{mmol}$ ) and $\mathrm{K}_{2} \mathrm{CO}_{3}(39 \mathrm{mg}, 0.28 \mathrm{mmol})$ at $80{ }^{\circ} \mathrm{C}$ for $24 \mathrm{~h}$. After solvent removal, the residue was purified by silica gel column chromatography (hexane) to afford the desired product $\mathbf{3 k}$ as a colourless solid (100 mg, $60 \%$ ).

Analytical data of 3k: Mp: $164-166{ }^{\circ} \mathrm{C}$. IR (neat) $v_{\max }: 2953,1735,1458,1284,797,692 \mathrm{~cm}^{-1} .{ }^{1} \mathrm{H}$ $\operatorname{NMR}\left(500 \mathrm{MHz}, \mathrm{CDCl}_{3}\right): \delta 7.95(\mathrm{~d}, J=8.0 \mathrm{~Hz}, 1 \mathrm{H}), 7.86(\mathrm{~d}, J=7.5 \mathrm{~Hz}, 2 \mathrm{H}), 7.81(\mathrm{~d}, J=8.0 \mathrm{~Hz}, 1 \mathrm{H})$, $7.63(\mathrm{~d}, J=7.5 \mathrm{~Hz}, 1 \mathrm{H}), 7.49$ (t, $J=7.0 \mathrm{~Hz}, 2 \mathrm{H}), 7.45(\mathrm{~d}, J=7.5 \mathrm{~Hz}, 1 \mathrm{H}), 7.38(\mathrm{q}, J=7.5 \mathrm{~Hz}, 3 \mathrm{H})$, 7.34-7.30 (m, 1H) ppm. ${ }^{13} \mathrm{C}\left\{{ }^{1} \mathrm{H}\right\}$ NMR $\left(125 \mathrm{MHz} \mathrm{CDCl}_{3}\right): \delta$ 156.0, 153.2, 142.1, 136.3, 128.9, 128.7, 128.0, 126.9, 125.2, 125.0, 124.9, 124.9, 124.7, 124.4, 123.8, 120.0, 118.8, 118.7 ppm. HRMS (ESI -Orbitrap) m/z: (M)+ calcd for $\mathrm{C}_{20} \mathrm{H}_{12} \mathrm{OS} 300.0603$, found 300.0600 . 


\section{6-chlorobenzothieno[3,2-b]benzofuran $(3 \mathrm{~m})$}

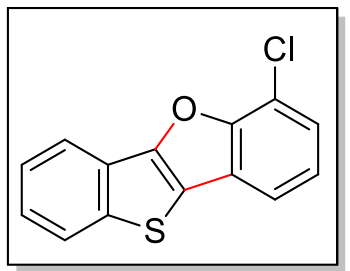

The reaction was performed according to the general procedure with 3-nitrobenzothiophene 1a (100 mg, $0.56 \mathrm{mmol}$ ), 2-chlorophenol $2 \mathrm{~m} \mathrm{(94} \mathrm{mg,} 0.73 \mathrm{mmol}$ ) and $\mathrm{K}_{2} \mathrm{CO}_{3}(39 \mathrm{mg}, 0.28 \mathrm{mmol}$ ) at $80{ }^{\circ} \mathrm{C}$ for $24 \mathrm{~h}$. After solvent removal, the residue was purified by silica gel column chromatography (hexane) to afford the desired product $3 \mathrm{~m}$ as a colourless solid (93 $\mathrm{mg}, 64 \%$ ).

Analytical data of $3 \mathrm{~m}$ : Mp: $138-140{ }^{\circ} \mathrm{C}$. IR (neat) $v_{\max }: 2916,1370,1181,1034,740,696 \mathrm{~cm}^{-1} .{ }^{1} \mathrm{H}$ NMR $\left(500 \mathrm{MHz}, \mathrm{CDCl}_{3}\right): \delta 8.02(\mathrm{~d}, J=8.0 \mathrm{~Hz}, 1 \mathrm{H}), 7.82(\mathrm{~d}, J=8.0 \mathrm{~Hz}, 1 \mathrm{H}), 7.56(\mathrm{~d}, J=7.5 \mathrm{~Hz}, 1 \mathrm{H})$, $7.43(\mathrm{t}, J=7.5 \mathrm{~Hz}, 1 \mathrm{H}), 7.37-7.32(\mathrm{~m}, 2 \mathrm{H}), 7.22(\mathrm{t}, J=7.0 \mathrm{~Hz}, 1 \mathrm{H}) \mathrm{ppm} .{ }^{13} \mathrm{C}\left\{{ }^{1} \mathrm{H}\right\} \mathrm{NMR}(125 \mathrm{MHz}$, $\left.\mathrm{CDCl}_{3}\right): \delta$ 154.3, 153.4, 142.2, 125.7, 125.4, 125.2, 125.1, 124.8, 124.4, 124.2, 120.1, 118.8, 118.1, 117.7 ppm. HRMS (ESI-Orbitrap) m/z: (M)+ calcd for $\mathrm{C}_{14} \mathrm{H}_{7} \mathrm{ClOS} 257.9901$, found 257.9903.

\section{6-bromobenzothieno[3,2-b]benzofuran (3n)}

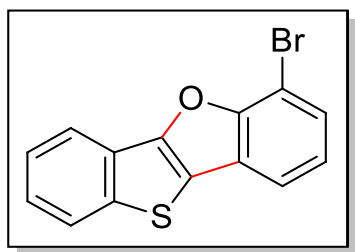

The reaction was performed according to the general procedure with 3-nitrobenzothiophene 1a (100 mg, $0.56 \mathrm{mmol}$ ), 2-bromophenol 2n (126 mg, $0.73 \mathrm{mmol}$ ) and $\mathrm{K}_{2} \mathrm{CO}_{3}(39 \mathrm{mg}, 0.28 \mathrm{mmol}$ ) at $80{ }^{\circ} \mathrm{C}$ for $24 \mathrm{~h}$. After solvent removal, the residue was purified by silica gel column chromatography (hexane) to afford the desired product $3 \mathrm{n}$ as a colourless solid (102 $\mathrm{mg}, 60 \%$ ).

Analytical data of $3 \mathrm{n}$ : Mp: $136-138{ }^{\circ} \mathrm{C}$. IR (neat) $v_{\max }: 2922,1730,1459,1211,967,739 \mathrm{~cm}^{-1} .{ }^{1} \mathrm{H}$ NMR $\left(500 \mathrm{MHz}, \mathrm{CDCl}_{3}\right): \delta 8.01(\mathrm{~d}, J=8.0 \mathrm{~Hz}, 1 \mathrm{H}), 7.80(\mathrm{~d}, J=8.5 \mathrm{~Hz}, 1 \mathrm{H}), 7.58(\mathrm{~d}, J=7.5 \mathrm{~Hz}, 1 \mathrm{H})$, $7.46(\mathrm{~d}, J=7.5 \mathrm{~Hz}, 1 \mathrm{H}), 7.42(\mathrm{t}, J=7.0 \mathrm{~Hz}, 1 \mathrm{H}), 7.34(\mathrm{t}, J=7.5 \mathrm{~Hz}, 1 \mathrm{H}), 7.18-7.13(\mathrm{~m}, 1 \mathrm{H}) \mathrm{ppm}$. ${ }^{13} \mathrm{C}\left\{{ }^{1} \mathrm{H}\right\} \mathrm{NMR}\left(125 \mathrm{MHz}, \mathrm{CDCl}_{3}\right): \delta 155.6,153.3,142.1,128.0,125.4,125.4,125.2,124.8,124.5$, 
124.4, 120.2, 119.0, 118.7, 105.0 ppm. HRMS (ESI-Orbitrap) m/z: $(\mathrm{M}+\mathrm{H})^{+}$calcd for $\mathrm{C}_{14} \mathrm{H}_{8} \mathrm{BrOS}$ 302.9474 , found 302.9475 .

\section{6-iodobenzothieno[3,2-b]benzofuran (3o)}<smiles></smiles>

The reaction was performed according to the general procedure with 3-nitrobenzothiophene 1a (100 mg, $0.56 \mathrm{mmol}$ ), 2-iodophenol 20 (161 mg, $0.73 \mathrm{mmol}$ ) and $\mathrm{K}_{2} \mathrm{CO}_{3}(39 \mathrm{mg}, 0.28 \mathrm{mmol}$ ) at 80 ${ }^{\circ} \mathrm{C}$ for $24 \mathrm{~h}$. After solvent removal, the residue was purified by silica gel column chromatography (hexane) to afford the desired product 30 as a colourless solid (114 mg, $58 \%$ ).

Analytical data of 3o: Mp: $136-138{ }^{\circ} \mathrm{C}$. IR (neat) $v_{\max }: 2921,1391,1219,1080,740,653 \mathrm{~cm}^{-1} .{ }^{1} \mathrm{H}$ $\operatorname{NMR}\left(500 \mathrm{MHz} \mathrm{CDCl}_{3}\right.$ ): $\delta 8.02(\mathrm{~d}, J=8.0 \mathrm{~Hz}, 1 \mathrm{H}), 7.81(\mathrm{~d}, J=8.5 \mathrm{~Hz}, 1 \mathrm{H}), 7.66(\mathrm{~d}, J=8.0 \mathrm{~Hz}, 1 \mathrm{H})$, $7.61(\mathrm{~d}, J=7.5 \mathrm{~Hz}, 1 \mathrm{H}), 7.42(\mathrm{t}, J=7.0 \mathrm{~Hz}, 1 \mathrm{H}), 7.34(\mathrm{t}, J=7.5 \mathrm{~Hz}, 1 \mathrm{H}), 7.03(\mathrm{t}, J=7.5 \mathrm{~Hz}, 1 \mathrm{H}) \mathrm{ppm}$. ${ }^{13} \mathrm{C}\left\{{ }^{1} \mathrm{H}\right\}$ NMR $\left(125 \mathrm{MHz}, \mathrm{CDCl}_{3}\right): \delta 158.7,153.0,142.1,133.9,125.4,125.1,125.0,124.9,124.3$, 120.2, 119.6, 119.2, 112.6, 76.0 ppm. HRMS (ESI-Orbitrap) m/z: (M)+calcd for $\mathrm{C}_{14} \mathrm{H}_{7} \mathrm{IOS} 349.9257$, found 349.9254 .

\section{6,9-dimethylbenzothieno[3,2-b]benzofuran (3p)}

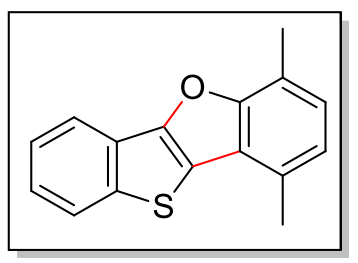

The reaction was performed according to the general procedure with 3-nitrobenzothiophene 1a (100 mg, $0.56 \mathrm{mmol}$ ), 2,5- dimethyl phenol 2p (89 mg, $0.73 \mathrm{mmol}$ ) and $\mathrm{K}_{2} \mathrm{CO}_{3}$ (39 mg, $0.28 \mathrm{mmol}$ ) at $80{ }^{\circ} \mathrm{C}$ for $24 \mathrm{~h}$. After solvent removal, the residue was purified by silica gel column chromatography (hexane) to afford the desired product $3 p$ as a colourless solid (78 $\mathrm{mg}, 55 \%$ ). 
Analytical data of 3p: Mp: $120-122^{\circ} \mathrm{C}$. IR (neat) $v_{\max }: 2921,1457,799,745,722,661 \mathrm{~cm}^{-1} .{ }^{1} \mathrm{H} \mathrm{NMR}$ (500 MHz, CDCl, $\mathrm{TMS}): \delta 7.98(\mathrm{~d}, J=7.5 \mathrm{~Hz}, 1 \mathrm{H}), 7.83$ (d, J=8.0 Hz, 1H), 7.42 (t, J = 7.5 Hz, 1H), $7.31(\mathrm{t}, J=7.0 \mathrm{~Hz}, 1 \mathrm{H}), 7.02(\mathrm{~d}, J=7.5 \mathrm{~Hz}, 1 \mathrm{H}), 6.97(\mathrm{~d}, J=7.0 \mathrm{~Hz}, 1 \mathrm{H}), 2.58(\mathrm{~s}, 3 \mathrm{H}), 2.53(\mathrm{~s}, 3 \mathrm{H})$ ppm. ${ }^{13} \mathrm{C}\left\{{ }^{1} \mathrm{H}\right\} \operatorname{NMR}\left(125 \mathrm{MHz}, \mathrm{CDCl}_{3}\right): \delta 157.5,152.2,142.4,127.5,126.1,125.3,124.8,124.4$, 124.3, 123.7, 123.5, 119.9, 119.6, 118.7, 18.9, 15.0 ppm. HRMS (ESI-Orbitrap) m/z: (M) ${ }^{+}$calcd for $\mathrm{C}_{16} \mathrm{H}_{12} \mathrm{OS} 252.0603$, found 252.0601 .

\section{6-bromo-8,9-difluorobenzothieno[3,2-b]benzofuran (3q)}

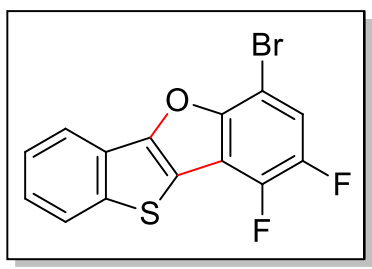

The reaction was performed according to the general procedure with 3-nitrobenzothiophene 1a (100 mg, $0.56 \mathrm{mmol}$ ), 2- bromo-4,5-difluorophenol 2q (153 mg, $0.73 \mathrm{mmol}$ ) and $\mathrm{K}_{2} \mathrm{CO}_{3}$ (39 mg, $0.28 \mathrm{mmol}$ ) at $80^{\circ} \mathrm{C}$ for $24 \mathrm{~h}$. After solvent removal, the residue was purified by silica gel column chromatography (hexane) to afford the desired product 3q as a colourless solid (134 mg, 71 \%).

Analytical data of 3q: Mp: $116-118^{\circ} \mathrm{C}$. IR (neat) $v_{\max }: 3035,1494,1253,860,800,661 \mathrm{~cm}^{-1} .{ }^{1} \mathrm{H}$ $\operatorname{NMR}\left(500 \mathrm{MHz}, \mathrm{CDCl}_{3}\right): \delta 7.95(\mathrm{~d}, J=7.5 \mathrm{~Hz}, 1 \mathrm{H}), 7.78(\mathrm{~d}, J=8.0 \mathrm{~Hz}, 1 \mathrm{H}), 7.41(\mathrm{t}, \mathrm{J}=7.0 \mathrm{~Hz}, 1 \mathrm{H})$, $7.35(\mathrm{t}, J=7.0 \mathrm{~Hz}, 1 \mathrm{H}), 7.28-7.24(\mathrm{~m}, 1 \mathrm{H}) \mathrm{ppm} .{ }^{13} \mathrm{C}\left\{{ }^{1} \mathrm{H}\right\} \mathrm{NMR}\left(125 \mathrm{MHz}, \mathrm{CDCl}_{3}\right): \delta$ 154.3, 152.2 (d, $J=7.4 \mathrm{~Hz}), 146.4\left(\mathrm{dd}, J_{1}=243.9 \mathrm{~Hz}, J_{2}=11.6 \mathrm{~Hz}\right), 142.9,141.8\left(\mathrm{dd}, J_{1}=251.1 \mathrm{~Hz}, J_{2}=7.3 \mathrm{~Hz}\right)$, 126.0, 125.3, 124.3, 124.0, 120.4, 116.3 (d, J = 23.5 Hz), 116.1 (d, J = 4.2 Hz), 115.6 (dd, $J_{1}=19.0$ $\left.\mathrm{Hz}, J_{2}=2.7 \mathrm{~Hz}\right), 98.5\left(\mathrm{dd}, J_{1}=9 \mathrm{~Hz}, J_{2}=3.8 \mathrm{~Hz}\right.$ ) ppm. HRMS (ESI- Orbitrap) m/z: $(\mathrm{M}+\mathrm{H})^{+}$calcd for $\mathrm{C}_{14} \mathrm{H}_{6} \mathrm{BrF}_{2} \mathrm{OS} 338.9285$, found 338.9282 .

3-bromobenzothieno[3,2-b]benzofuran (3r)<smiles>Brc1ccc2sc3c4ccccc4oc3c2c1</smiles> 
The reaction was performed according to the general procedure with 5-bromo-3nitrobenzothiophene $\mathbf{1 b}$ (100 mg, $0.39 \mathrm{mmol})$, phenol $\mathbf{2 b}(48 \mathrm{mg}, 0.51 \mathrm{mmol})$ and $\mathrm{K}_{2} \mathrm{CO}_{3}(26 \mathrm{mg}$, $0.19 \mathrm{mmol}$ ) at $80^{\circ} \mathrm{C}$ for $24 \mathrm{~h}$. After solvent removal, the residue was purified by silica gel column chromatography (hexane) to afford the desired product $3 r$ as a colourless solid (83 $\mathrm{mg}, 70 \%$ ).

Analytical data of 3r: Mp: 122- $124^{\circ} \mathrm{C}$. IR (neat) $v_{\max }$ : 2919, 1687, 1436, 1182, 1052, 793, $730 \mathrm{~cm}^{-}$ 1. ${ }^{1} \mathrm{H}$ NMR $\left(500 \mathrm{MHz}, \mathrm{CDCl}_{3}\right): \delta 8.07(\mathrm{~s}, 1 \mathrm{H}), 7.67(\mathrm{~d}, J=8.0 \mathrm{~Hz}, 2 \mathrm{H}), 7.58(\mathrm{~d}, J=8.0 \mathrm{~Hz}, 1 \mathrm{H}), 7.41(\mathrm{~d}$, $J=9.0 \mathrm{~Hz}, 1 \mathrm{H}), 7.34(\mathrm{t}, J=7.5 \mathrm{~Hz}, 1 \mathrm{H}), 7.29(\mathrm{t}, J=7.5 \mathrm{~Hz}, 1 \mathrm{H}) \mathrm{ppm} .{ }^{13} \mathrm{C}\left\{{ }^{1} \mathrm{H}\right\} \mathrm{NMR}\left(125 \mathrm{MHz}, \mathrm{CDCl}_{3}\right)$ : $\delta 158.9,151.7,140.5,127.8,126.6,125.6,125.5,123.7,123.5,122.5,120.3,119.8,119.0,112.7$ ppm. HRMS (ESI-Orbitrap) m/z: $(\mathrm{M}+\mathrm{H})^{+}$calcd for $\mathrm{C}_{14} \mathrm{H}_{8} \mathrm{BrOS} 302.9474$, found 302.9477 .

\section{3-bromo-8-methylbenzothieno[3,2-b]benzofuran (3s)}

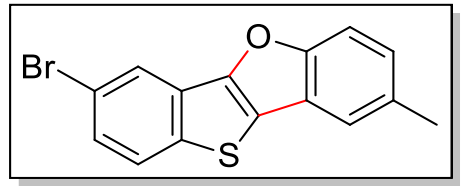

The reaction was performed according to the general procedure with 5-bromo-3nitrobenzothiophene $\mathbf{1 b}(100 \mathrm{mg}, 0.39 \mathrm{mmol}), p$-cresol 2c $(55 \mathrm{mg}, 0.51 \mathrm{mmol})$ and $\mathrm{K}_{2} \mathrm{CO}_{3}(26 \mathrm{mg}$, $0.19 \mathrm{mmol}$ ) at $80^{\circ} \mathrm{C}$ for $24 \mathrm{~h}$. After solvent removal, the residue was purified by silica gel column chromatography (hexane) to afford the desired product 3 s as a colourless solid (95 $\mathrm{mg}, 77 \%$ ).

Analytical data of 3s: Mp: $94-96{ }^{\circ} \mathrm{C}$. IR (neat) $v_{\max }: 2916,1455,1372,833,789,669 \mathrm{~cm}^{-1} .{ }^{1} \mathrm{H}$ NMR (500 MHz, CDCl 3 ): $\delta 8.03(\mathrm{~s}, 1 \mathrm{H}), 7.62(\mathrm{~d}, J=8.5 \mathrm{~Hz}, 1 \mathrm{H}), 7.43(\mathrm{~s}, 2 \mathrm{H}), 7.37(\mathrm{~d}, J=8.5 \mathrm{~Hz}, 1 \mathrm{H}), 7.13$ (d, J = 8.5 Hz, 1H), 2.42 (s, 3H) ppm. ${ }^{13} \mathrm{C}\left\{{ }^{1} \mathrm{H}\right\} \mathrm{NMR}\left(125 \mathrm{MHz}, \mathrm{CDCl}_{3}\right): \delta$ 157.3, 151.8, 140.4, 133.2, 127.6, 126.7, 126.6, 125.6, 123.7, 122.4, 120.1, 119.7, 118.9, 112.2, 21.4 ppm. HRMS (ESIOrbitrap) m/z: (M)+ calcd for $\mathrm{C}_{15} \mathrm{H}_{9} \mathrm{BrOS} 315.9552$, found 315.9553 .

\section{3,8-dibromobenzothieno[3,2-b]benzofuran (3t)}

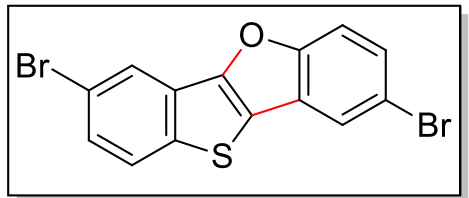


The reaction was performed according to the general procedure with 5-bromo-3nitrobenzothiophene $\mathbf{1 b}$ (100 mg, $0.39 \mathrm{mmol})$, 4-bromophenol $2 \mathrm{~g}(87 \mathrm{mg}, 0.51 \mathrm{mmol})$ and $\mathrm{K}_{2} \mathrm{CO}_{3}$ ( $26 \mathrm{mg}, 0.19 \mathrm{mmol}$ ) at $80^{\circ} \mathrm{C}$ for $24 \mathrm{~h}$. After solvent removal, the residue was purified by silica gel column chromatography (hexane) to afford the desired product $3 \mathrm{t}$ as a colourless solid (66 $\mathrm{mg}$, $68 \%)$.

Analytical data of 3t: Mp: $194-196{ }^{\circ} \mathrm{C}$. IR (neat) $v_{\max }: 2921,1586,1433,1058,917,833,795 \mathrm{~cm}^{-1}$. ${ }^{1} \mathrm{H} \mathrm{NMR}\left(500 \mathrm{MHz}, \mathrm{CDCl}_{3}\right): \delta 8.07(\mathrm{~s}, 1 \mathrm{H}), 7.81(\mathrm{~s}, 1 \mathrm{H}), 7.66(\mathrm{~d}, J=8.5 \mathrm{~Hz}, 1 \mathrm{H}), 7.47-7.43(\mathrm{~m}, 3 \mathrm{H})$ ppm. ${ }^{13} \mathrm{C}\left\{{ }^{1} \mathrm{H}\right\} \operatorname{NMR}\left(125 \mathrm{MHz}, \mathrm{CDCl}_{3}\right): \delta 157.6,140.7,128.4,128.3,126.2,125.7,125.5,122.7$, 122.5, 120.1, 119.3, 119.2, 116.6, 114.1 ppm. HRMS (ESI-Orbitrap) m/z: (M)+calcd for $\mathrm{C}_{14} \mathrm{H}_{6} \mathrm{Br}_{2} \mathrm{OS}$ 379.8501 , found 379.8503 .

3,-bromo-8-iodobenzothieno[3,2-b]benzofuran (3u)

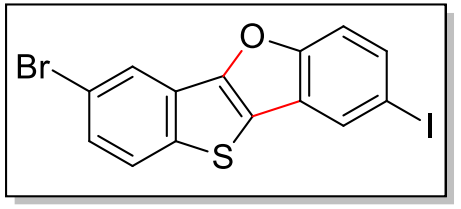

The reaction was performed according to the general procedure with 5-bromo-3nitrobenzothiophene $\mathbf{1 b}(100 \mathrm{mg}, 0.39 \mathrm{mmol})$, 4-iodophenol $2 \mathrm{r}(112 \mathrm{mg}, 0.51 \mathrm{mmol})$ and $\mathrm{K}_{2} \mathrm{CO}_{3}$ (26 mg, $0.19 \mathrm{mmol}$ ) at $80^{\circ} \mathrm{C}$ for $24 \mathrm{~h}$. After solvent removal, the residue was purified by silica gel column chromatography (hexane) to afford the desired product $\mathbf{3 u}$ as a colourless solid (73 $\mathrm{mg}$, $62 \%)$.

Analytical data of $3 \mathrm{u}: \mathrm{Mp}: 188-190^{\circ} \mathrm{C}$. IR (neat) $\mathrm{v}_{\max }: 2919,1588,1437,1190,915,863,700 \mathrm{~cm}^{-}$ 1. ${ }^{1} \mathrm{H} \mathrm{NMR}\left(500 \mathrm{MHz}, \mathrm{CDCl}_{3}\right) \delta 8.07(\mathrm{~s}, 1 \mathrm{H}), 8.02(\mathrm{~s}, 1 \mathrm{H}), 7.66(\mathrm{~d}, J=8.5 \mathrm{~Hz}, 1 \mathrm{H}), 7.61(\mathrm{~d}, J=8.0 \mathrm{~Hz}$, $1 \mathrm{H}), 7.43(\mathrm{~d}, J=9.0 \mathrm{~Hz}, 1 \mathrm{H}), 7.35(\mathrm{~d}, J=8.5 \mathrm{~Hz}, 1 \mathrm{H}) \mathrm{ppm} .{ }^{13} \mathrm{C}\left\{{ }^{1} \mathrm{H}\right\} \mathrm{NMR}\left(125 \mathrm{MHz}, \mathrm{CDCl}_{3}\right): \delta 157.3$, $151.3,139.7,133.0,127.6,127.3,125.1,125.1,124.6,124.5,121.7,118.8,118.1,113.6$ ppm. HRMS (ESI-Orbitrap) m/z: (M) ${ }^{+}$calcd for $\mathrm{C}_{14} \mathrm{H}_{6} \mathrm{BrIOS} 427.8362$, found 427.8365 . 


\section{8-methoxy-4b,9b-dihydrobenzothieno[3,2-b]benzofuran-3-carbonitrile (3v)}

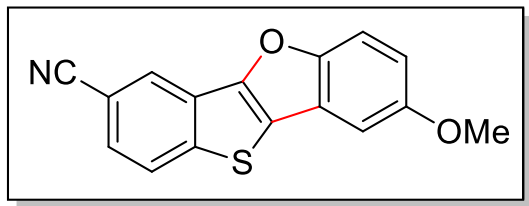

The reaction was performed according to the general procedure with 3-nitrobenzothiophene-5carbonitrile 1c (100 mg, $0.49 \mathrm{mmol})$, 4-methoxy phenol 2a (79 mg, $0.64 \mathrm{mmol})$ and $\mathrm{K}_{2} \mathrm{CO}_{3}(34 \mathrm{mg}$, $0.24 \mathrm{mmol}$ ) at $80^{\circ} \mathrm{C}$ for $24 \mathrm{~h}$. After solvent removal, the residue was purified by silica gel column chromatography (10\% ethyl acetate in hexane) to afford the desired product $\mathbf{3} \mathbf{v}$ as a colourless solid (96 mg, $70 \%)$.

Analytical data of 3v: Mp: 170-172 ${ }^{\circ} \mathrm{C}$. IR (neat) $v_{\max }: 2923,1589,1448,1215,1030,821,795 \mathrm{~cm}^{-}$ 1. ${ }^{1} \mathrm{H} \mathrm{NMR}\left(500 \mathrm{MHz}, \mathrm{CDCl}_{3}\right): \delta 8.16(\mathrm{~s}, 1 \mathrm{H}), 7.87(\mathrm{~d}, J=8.5 \mathrm{~Hz}, 1 \mathrm{H}), 7.48(\mathrm{t}, J=8.0 \mathrm{~Hz}, 2 \mathrm{H}), 7.09$ (s, $1 \mathrm{H}), 6.95(\mathrm{~d}, J=9.5 \mathrm{~Hz}, 1 \mathrm{H}), 3.83(\mathrm{~s}, 3 \mathrm{H}) \mathrm{ppm} .{ }^{13} \mathrm{C}\left\{{ }^{1} \mathrm{H}\right\} \mathrm{NMR}\left(125 \mathrm{MHz}, \mathrm{CDCl}_{3}\right): \delta$ 156.5, 153.9, $152.4,145.6,126.7,125.2,125.1,123.8,123.7,120.9,119.0,114.6,113.4,108.7,102.4,56.0$ ppm. HRMS (ESI-Orbitrap) m/z: $(\mathrm{M}+\mathrm{H})^{+}$calcd for $\mathrm{C}_{16} \mathrm{H}_{11} \mathrm{NO}_{2} \mathrm{~S} 280.0427$, found 280.0436 .

\section{6-isopropyl-9-methylbenzothieno[3,2-b]benzofuran (3w)}

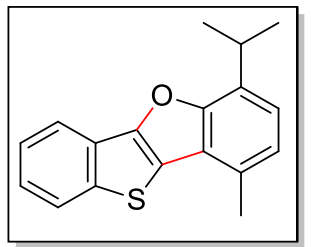

The reaction was performed according to the general procedure with 3-nitrobenzothiophene 1a (100 mg, $0.56 \mathrm{mmol})$, thymol $2 \mathrm{~s}(111 \mathrm{mg}, 0.73 \mathrm{mmol})$ and $\mathrm{K}_{2} \mathrm{CO}_{3}(39 \mathrm{mg}, 0.28 \mathrm{mmol})$ at $80{ }^{\circ} \mathrm{C}$ for $24 \mathrm{~h}$. After solvent removal, the residue was purified by silica gel column chromatography (hexane) to afford the desired product $3 w$ as a colourless solid (97 mg, $62 \%$ ).

Analytical data of $3 w$ : Mp: $90-92^{\circ} \mathrm{C}$. IR (neat) $v_{\max }: 2922,1733,1460,1375,1080,968,820 \mathrm{~cm}^{-1}$. ${ }^{1} \mathrm{H} \mathrm{NMR}\left(500 \mathrm{MHz}, \mathrm{CDCl}_{3}\right): \delta 7.98(\mathrm{~d}, J=8.0 \mathrm{~Hz}, 1 \mathrm{H}), 7.82(\mathrm{~d}, J=8.5 \mathrm{~Hz}, 1 \mathrm{H}), 7.41(\mathrm{t}, J=7.5 \mathrm{~Hz}, 1 \mathrm{H})$, 
$7.31(\mathrm{t}, J=7.5 \mathrm{~Hz}, 1 \mathrm{H}), 7.09$ (d, J= $7.5 \mathrm{~Hz}, 1 \mathrm{H}), 7.02$ (d, J= $7.5 \mathrm{~Hz}, 1 \mathrm{H}), 3.54-3.48(\mathrm{~m}, 1 \mathrm{H}), 2.58$ (s, 3H), $1.37(\mathrm{~d}, J=7.0 \mathrm{~Hz}, 6 \mathrm{H}) \mathrm{ppm} .{ }^{13} \mathrm{C}\left\{{ }^{1} \mathrm{H}\right\} \mathrm{NMR}\left(125 \mathrm{MHz}, \mathrm{CDCl}_{3}\right): \delta 155.5,151.1,141.4,130.0$, $126.5,124.3,123.8,123.4,123.3,122.9,122.7,121.1,118.6,117.6,27.4,21.8,17.9$ ppm. HRMS (ESI-Orbitrap) m/z: $(\mathrm{M}+\mathrm{H})^{+}$calcd for $\mathrm{C}_{18} \mathrm{H}_{17} \mathrm{OS} 281.0997$, found 281.0992.

\section{8-allyl-6-methoxybenzothieno[3,2-b]benzofuran (3x)}

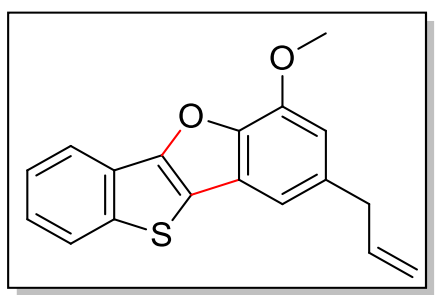

The reaction was performed according to the general procedure with 3-nitrobenzothiophene 1a (100 mg, $0.56 \mathrm{mmol})$, eugenol $2 \mathrm{t}(120 \mathrm{mg}, 0.73 \mathrm{mmol})$ and $\mathrm{K}_{2} \mathrm{CO}_{3}(39 \mathrm{mg}, 0.28 \mathrm{mmol})$ at $80{ }^{\circ} \mathrm{C}$ for $24 \mathrm{~h}$. After solvent removal, the residue was purified by silica gel column chromatography (hexane) to afford the desired product $3 \mathbf{x}$ as a colourless solid (106 mg, $64 \%$ ).

Analytical data of 3x: Mp: $88-90^{\circ} \mathrm{C}$. IR (neat) $v_{\max }: 2921,1591,1342,1134,1021,908,750 \mathrm{~cm}^{-1}$.

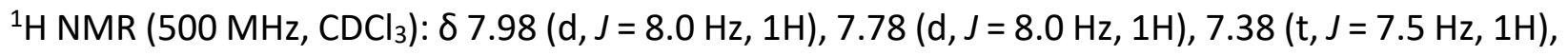
$7.29(\mathrm{t}, J=7.5 \mathrm{~Hz}, 1 \mathrm{H}), 7.07(\mathrm{~s}, 1 \mathrm{H}), 6.68(\mathrm{~s}, 1 \mathrm{H}), 6.01-5.93(\mathrm{~m}, 1 \mathrm{H}), 5.10-5.04(\mathrm{~m}, 2 \mathrm{H}), 4.00(\mathrm{~s}, 3 \mathrm{H})$, $3.44(\mathrm{~d}, J=6.5 \mathrm{~Hz}, 2 \mathrm{H}) \mathrm{ppm} .{ }^{13} \mathrm{C}\left\{{ }^{1} \mathrm{H}\right\} \mathrm{NMR}\left(125 \mathrm{MHz}, \mathrm{CDCl}_{3}\right): \delta 152.2,145.4,144.5,141.0,136.5$, $135.3,124.4,124.1,123.9,123.8,123.3,118.8,117.8,115.0,110.5$, 107.2, 55.2, 39.5 ppm. HRMS (ESI-Orbitrap) m/z: (M)+ calcd for $\mathrm{C}_{18} \mathrm{H}_{14} \mathrm{O}_{2} \mathrm{~S} 294.0709$, found 294.0712 .

\section{Benzothieno[3,2-b]naphtha[2,1-d]furan $(3 y)^{5}$}

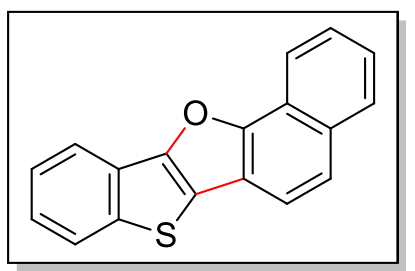

The reaction was performed according to the general procedure with 3-nitrobenzothiophene 1a (100 mg, $0.56 \mathrm{mmol}$ ), 1-naphthol $2 \mathrm{u}(105 \mathrm{mg}, 0.73 \mathrm{mmol})$ and $\mathrm{K}_{2} \mathrm{CO}_{3}(39 \mathrm{mg}, 0.28 \mathrm{mmol})$ at $80{ }^{\circ} \mathrm{C}$ 
for $24 \mathrm{~h}$. After solvent removal, the residue was purified by silica gel column chromatography (hexane) to afford the desired product $3 y$ as a colourless solid (100 mg, $65 \%$ ).

Analytical data of $3 y$ : Mp: $144-146^{\circ} \mathrm{C}$. IR (neat) $\mathrm{V}_{\max }$ : 3027, 1409, 1256, 866, $744 \mathrm{~cm}^{-1} .{ }^{1} \mathrm{H}$ NMR (500 MHz, CDCl, $\mathrm{TMS}): \delta 8.38(\mathrm{~d}, J=8.0 \mathrm{~Hz}, 1 \mathrm{H}), 8.02(\mathrm{~d}, J=8.0 \mathrm{~Hz}, 1 \mathrm{H}), 7.89(\mathrm{~d}, J=8.0 \mathrm{~Hz}, 1 \mathrm{H})$, $7.82(\mathrm{~d}, J=8.0 \mathrm{~Hz}, 1 \mathrm{H}), 7.72-7.67(\mathrm{~m}, 2 \mathrm{H}), 7.57(\mathrm{t}, J=7.5 \mathrm{~Hz}, 1 \mathrm{H}), 7.47-7.41(\mathrm{~m}, 2 \mathrm{H}), 7.31(\mathrm{t}, J=$ $7.5 \mathrm{~Hz}, 1 \mathrm{H}) \mathrm{ppm} .{ }^{13} \mathrm{C}\left\{{ }^{1} \mathrm{H}\right\} \mathrm{NMR}\left(125 \mathrm{MHz}, \mathrm{CDCl}_{3}\right): \delta 154.2,152.5,141.7,131.6,128.5,126.7,125.5$, $125.3,125.0,124.6,124.4,124.0,122.1,120.2,119.9,119.6,119.5,118.0$ ppm.

\section{Benzothieno[3,2-b]naphtha[2,3- $d]$ furan $(3 z)^{3}$}

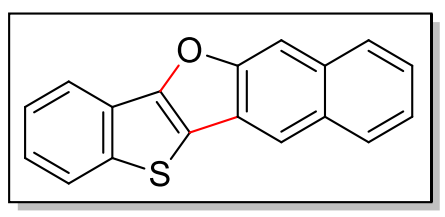

The reaction was performed according to the general procedure with 3-nitrobenzothiophene 1a (100 mg, $0.56 \mathrm{mmol}$ ), 2-naphthol $2 \mathrm{v}\left(105 \mathrm{mg}, 0.73 \mathrm{mmol}\right.$ ) and $\mathrm{K}_{2} \mathrm{CO}_{3}\left(39 \mathrm{mg}, 0.28 \mathrm{mmol}\right.$ ) at $80^{\circ} \mathrm{C}$ for $24 \mathrm{~h}$. After solvent removal, the residue was purified by silica gel column chromatography (hexane) to afford the desired product $3 z$ as a colourless solid (100 $\mathrm{mg}, 65 \%)$.

Analytical data of 3z: Mp: $194-196{ }^{\circ} \mathrm{C}$. IR (neat) $v_{\max }: 2995,1674,1459,1254,744,684 \mathrm{~cm}^{-1} .{ }^{1} \mathrm{H}$ NMR (500 MHz, CDCl $3, T M S): \delta 8.07(d, J=6.5 \mathrm{~Hz}, 1 \mathrm{H}), 7.97(\mathrm{~d}, J=6.0 \mathrm{~Hz}, 1 \mathrm{H}$ ), $7.91(\mathrm{~d}, J=7.5 \mathrm{~Hz}$, $1 \mathrm{H}), 7.85(\mathrm{~d}, J=5.0 \mathrm{~Hz}, 1 \mathrm{H}), 7.74-7.70(\mathrm{~m}, 2 \mathrm{H}), 7.62-7.59(\mathrm{~m}, 1 \mathrm{H}), 7.48-7.45(\mathrm{~m}, 1 \mathrm{H}), 7.43-7.40$ (m, $1 \mathrm{H}), 7.32-7.30(\mathrm{~m}, 1 \mathrm{H}) \mathrm{ppm} .{ }^{13} \mathrm{C}\left\{{ }^{1} \mathrm{H}\right\} \mathrm{NMR}\left(125 \mathrm{MHz}, \mathrm{CDCl}_{3}\right): \delta 156.2,152.4,142.3,130.5$, $128.7,126.9,126.6,125.9,125.0,124.9,124.6,124.6,124.4,124.2,119.5,118.9,118.8,113.2$ ppm.

\section{9-Bromobenzothieno[3,2-b]naphtha[2,3-d]furan (3aa)}

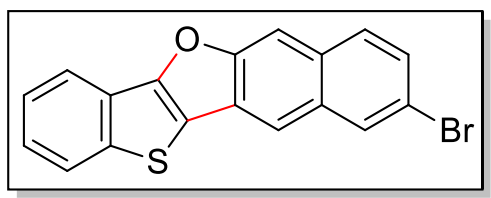


The reaction was performed according to the general procedure with 3-nitrobenzothiophene 1a (100 mg, $0.56 \mathrm{mmol}$ ), 6-bromo-2-naphthol $2 \mathrm{w}$ (163 mg, $0.73 \mathrm{mmol}$ ) and $\mathrm{K}_{2} \mathrm{CO}_{3}(39 \mathrm{mg}, 0.28$ $\mathrm{mmol}$ ) at $80^{\circ} \mathrm{C}$ for $24 \mathrm{~h}$. After solvent removal, the residue was purified by silica gel column chromatography (hexane) to afford the desired product 3aa as a colourless solid (137 mg, $69 \%$ ).

Analytical data of 3aa: Mp: 196-198 ${ }^{\circ} \mathrm{C}$. IR (neat) $\mathrm{V}_{\max }$ : 2916, 1733, 1459, 798, $673 \mathrm{~cm}^{-1} .{ }^{1} \mathrm{H}$ NMR (500 MHz, CDCl $3, \mathrm{TMS}): \delta 8.34(\mathrm{~d}, J=8.0 \mathrm{~Hz}, 1 \mathrm{H}), 7.97(\mathrm{~d}, J=8.0 \mathrm{~Hz}, 1 \mathrm{H}), 7.84(\mathrm{~d}, J=8.0 \mathrm{~Hz}, 1 \mathrm{H})$, $7.77(\mathrm{~d}, J=8.0 \mathrm{~Hz}, 1 \mathrm{H}), 7.64(\mathrm{~d}, J=3.0 \mathrm{~Hz}, 1 \mathrm{H}), 7.53(\mathrm{t}, J=7.5 \mathrm{~Hz}, 1 \mathrm{H}), 7.42-7.36(\mathrm{~m}, 2 \mathrm{H}), 7.26(\mathrm{t}$, $J=7.5 \mathrm{~Hz}, 1 \mathrm{H}) \mathrm{ppm} .{ }^{13} \mathrm{C}\left\{{ }^{1} \mathrm{H}\right\} \mathrm{NMR}\left(125 \mathrm{MHz}, \mathrm{CDCl}_{3}\right): \delta 154.2,152.5,141.7,131.6,128.5,126.7$, $125.5,125.3,125.0,124.6,124.4,124.0,122.1,120.2$, 119.9, 119.6, 119.5, 118.0 ppm. HRMS (ESIOrbitrap) m/z: (M) ${ }^{+}$calcd for $\mathrm{C}_{18} \mathrm{H}_{9} \mathrm{BrOS} 351.9552$, found 351.9553 .

3-bromobenzothieno[3,2-b]naphtha[2,3-d]furan (3ab)

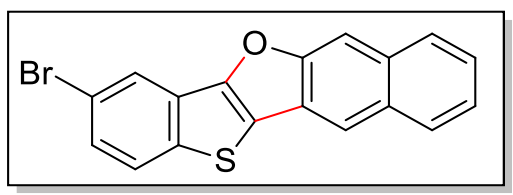

The reaction was performed according to the general procedure with 5-bromo-3nitrobenzothiophene $\mathbf{1 b}$ (100 mg, $0.39 \mathrm{mmol})$, 2-naphthol $2 \mathbf{v}(74 \mathrm{mg}, 0.51 \mathrm{mmol})$ and $\mathrm{K}_{2} \mathrm{CO}_{3}(26$ $\mathrm{mg}, 0.19 \mathrm{mmol}$ ) at $80^{\circ} \mathrm{C}$ for $24 \mathrm{~h}$. After solvent removal, the residue was purified by silica gel column chromatography (hexane) to afford the desired product 3ab as a colourless solid (94 mg, $68 \%)$.

Analytical data of 3ab: Mp: 194-196 ${ }^{\circ} \mathrm{C}$. IR (neat) $v_{\max }: 2920,1336,1205,1054,971,790,730 \mathrm{~cm}^{-}$ 1. ${ }^{1} \mathrm{H}$ NMR (500 MHz, $\left.\mathrm{CDCl}_{3}\right): \delta 8.10(\mathrm{~s}, 1 \mathrm{H}), 8.05(\mathrm{~d}, J=8.0 \mathrm{~Hz}, 1 \mathrm{H}), 7.93(\mathrm{~d}, J=8.0 \mathrm{~Hz}, 1 \mathrm{H}), 7.77$ (d, $J=9.0 \mathrm{~Hz}, 1 \mathrm{H}), 7.72-7.69(\mathrm{~m}, 2 \mathrm{H}), 7.62(\mathrm{t}, J=7.5 \mathrm{~Hz}, 1 \mathrm{H}), 7.49(\mathrm{t}, J=7.5 \mathrm{~Hz}, 1 \mathrm{H}), 7.40(\mathrm{~d}, J=$ $8.5 \mathrm{~Hz}, 1 \mathrm{H}) \mathrm{ppm} .{ }^{13} \mathrm{C}\left\{{ }^{1} \mathrm{H}\right\} \mathrm{NMR}\left(125 \mathrm{MHz}, \mathrm{CDCl}_{3}\right): \delta 156.5,151.0,140.7,130.4,128.8,127.4,127.1$, 126.6, 126.4, 125.6, 125.2, 124.1, 122.2, 120.4, 119.2, 119.1, 113.2 ppm. HRMS (ESI-Orbitrap) $\mathrm{m} / \mathrm{z}$ : (M) ${ }^{+}$calcd for $\mathrm{C}_{18} \mathrm{H}_{9} \mathrm{BrOS} 351.9552$, found 351.9557 .

\section{2-methoxybenzothieno[2,3-b]benzofuran (5)}




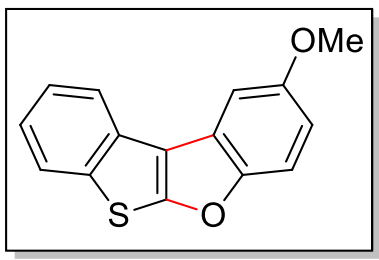

The reaction was performed according to the general procedure with 2-nitrobenzothiophene 4 (100 mg, $0.56 \mathrm{mmol})$, 4- methoxy phenol 2a (91 mg, $0.73 \mathrm{mmol}$ ) and $\mathrm{K}_{2} \mathrm{CO}_{3}$ (39 mg, $0.28 \mathrm{mmol}$ ) at $80{ }^{\circ} \mathrm{C}$ for $24 \mathrm{~h}$. After solvent removal, the residue was purified by silica gel column chromatography (hexane) to afford the desired product 5 as an yellow liquid (54 mg, 38\%).

Analytical data of 5: ${ }^{1} \mathrm{H} \mathrm{NMR}\left(500 \mathrm{MHz}, \mathrm{CDCl}_{3}, \mathrm{TMS}\right): \delta 7.95(\mathrm{~d}, J=8.0 \mathrm{~Hz}, 1 \mathrm{H}), 7.74(\mathrm{~d}, J=8.0 \mathrm{~Hz}$, $1 \mathrm{H}), 7.44-7.41(\mathrm{~m}, 2 \mathrm{H}), 7.30(\mathrm{~d}, J=2.5 \mathrm{~Hz}, 1 \mathrm{H}), 7.27(\mathrm{t}, J=7.5 \mathrm{~Hz}, 1 \mathrm{H}), 6.84\left(\mathrm{dd}, J_{1}=9.0 \mathrm{~Hz}, J_{2}=\right.$ $2.5 \mathrm{~Hz} 1 \mathrm{H}), 3.86$ (s, 3H) ppm. ${ }^{13} \mathrm{C}\left\{{ }^{1} \mathrm{H}\right\} \operatorname{NMR}\left(125 \mathrm{MHz}, \mathrm{CDCl}_{3}\right): \delta$ 158.8, 155.4, 154.5, 137.1, 129.4, 124.3, 123.5, 123.0, 122.7, 120.3, 118.9, 111.2, 110.0, 101.8, 55.0 ppm. HRMS (ESI-Orbitrap) m/z: $(\mathrm{M}+\mathrm{H})^{+}$calcd for $\mathrm{C}_{15} \mathrm{H}_{11} \mathrm{O}_{2} \mathrm{~S} 255.0474$, found 255.0476 .

\section{Procedure for scale up of $3 a$}

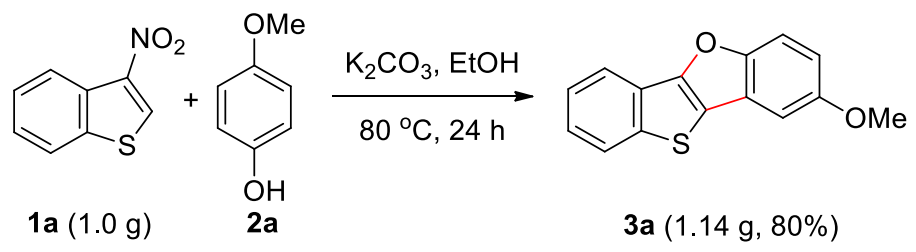

A mixture of 3-nitrobenzothiophene 1a (1.0 g, $5.59 \mathrm{mmol})$, 4-methoxyphenol 2a (901 mg, 7.26 $\mathrm{mmol}$ ) and $\mathrm{K}_{2} \mathrm{CO}_{3}(386 \mathrm{mg}, 2.79 \mathrm{mmol}$ ) was weighed into a dry reaction tube. Distilled EtOH (20 $\mathrm{mL}$ ) was added and allowed to stir at $80^{\circ} \mathrm{C}$ on an oil bath for 24 hours. After completion of the reaction as indicated from the TLC, the solvent was removed under vacuum. The residue was then purified by column chromatography (silica gel, eluent: hexane) to afford $3 a(1.14 \mathrm{~g}, 80 \%)$. 


\section{Procedure for the synthesis of benzofuro[3,2-b]indole by the annulation of electrophilic indole}

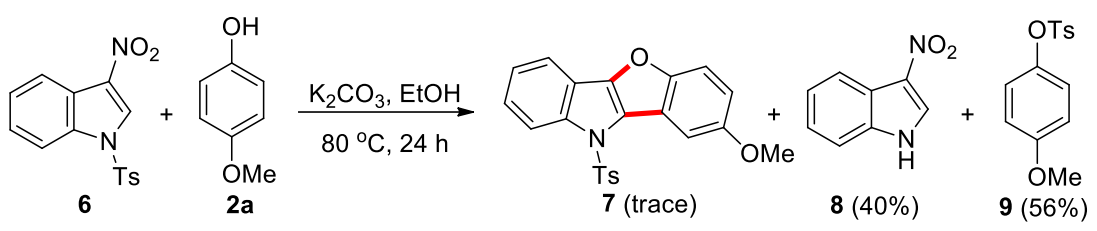

The reaction was performed according to the general procedure with $N$-Ts-3-nitro-indole 6 (100 $\mathrm{mg}, 0.32 \mathrm{mmol})$, 4- methoxy phenol $2 \mathrm{a}(51 \mathrm{mg}, 0.41 \mathrm{mmol})$ and $\mathrm{K}_{2} \mathrm{CO}_{3}(22 \mathrm{mg}, 0.16 \mathrm{mmol})$ at 80 ${ }^{\circ} \mathrm{C}$ for $24 \mathrm{~h}$. After completion of the reaction as indicated from the TLC, the solvent was removed under vacuum. The residue was then purified by column chromatography (silica gel, eluent: mixtures of ethyl acetate in hexane) to afford 7 (traces), 8 (21 mg, $40 \%$ ) as a pale yellow solid and 9 (49 $\mathrm{mg}, 56 \%)$ as a colourless solid.

Analytical data of 7: Mp: $132-134{ }^{\circ} \mathrm{C}$. IR (neat) $v_{\max }: 2923,1449,1372,1239,1180,921,808,752$, $698 \mathrm{~cm}^{-1} .{ }^{1} \mathrm{H}$ NMR $\left(500 \mathrm{MHz}, \mathrm{CDCl}_{3}, \mathrm{TMS}\right): \delta 8.20(\mathrm{~d}, J=8.5 \mathrm{~Hz}, 1 \mathrm{H}), 7.82(\mathrm{~s}, 1 \mathrm{H}), 7.60-7.56(\mathrm{~m}$, $3 \mathrm{H}), 7.42(\mathrm{~d}, J=9 \mathrm{~Hz}, 1 \mathrm{H}), 7.32(\mathrm{t}, J=7.5 \mathrm{~Hz}, 1 \mathrm{H}), 7.27(\mathrm{t}, J=7.5 \mathrm{~Hz}, 1 \mathrm{H}), 7.01(\mathrm{~d}, J=8 \mathrm{~Hz}, 2 \mathrm{H})$ $6.93(\mathrm{~d}, J=9 \mathrm{~Hz}, 1 \mathrm{H}), 3.91(\mathrm{~s}, 3 \mathrm{H}), 2.19(\mathrm{~s}, 3 \mathrm{H}) \mathrm{ppm} .{ }^{13} \mathrm{C}\left\{{ }^{1} \mathrm{H}\right\} \mathrm{NMR}\left(125 \mathrm{MHz}, \mathrm{CDCl}_{3}\right): \delta 156.6,154.2$, 147.6, 144.9, 139.6, 134.1, 129.8, 126.8, 125.4, 124.4, 119.7, 118.7, 117.5, 115.9, 113.5, 113.0, 103.2, 103.1, 56.1, 21.5 ppm. HRMS (ESI-Orbitrap) m/z: $(\mathrm{M}+\mathrm{H})^{+}$calcd for $\mathrm{C}_{22} \mathrm{H}_{17} \mathrm{NO}_{4} \mathrm{~S} 392.0951$, found 392.0954 .

Analytical data of 8 $^{1 \mathrm{a}}:{ }^{1} \mathrm{H}$ NMR (500 MHz, DMSO-d 6 , TMS): $\delta 12.66(\mathrm{~s}, 1 \mathrm{H}), 8.66(\mathrm{~s}, 1 \mathrm{H}), 8.10(\mathrm{~d}, J$ $=7.5 \mathrm{~Hz}, 1 \mathrm{H}), 7.58(\mathrm{~d}, J=7.5 \mathrm{~Hz}, 1 \mathrm{H}), 7.39-7.33(\mathrm{~m}, 2 \mathrm{H}) \mathrm{ppm} .{ }^{13} \mathrm{C}\left\{{ }^{1} \mathrm{H}\right\}$ NMR $\left(125 \mathrm{MHz}, \mathrm{DMSO}-\mathrm{d}_{6}\right)$ : $\delta 135.5,131.0,128.9,124.6,124.2,120.3,119.9,113.8$ ppm. HRMS (ESI-Orbitrap) m/z: $(M+H)^{+}$ calcd for $\mathrm{C}_{8} \mathrm{H}_{7} \mathrm{~N}_{2} \mathrm{O}_{2}$ 163.0502, found 163.0503 .

Analytical data of $9^{6}:{ }^{1} \mathrm{H}$ NMR (500 MHz, $\left.\mathrm{CDCl}_{3}, \mathrm{TMS}\right): \delta 7.61(\mathrm{~d}, J=8.0 \mathrm{~Hz}, 2 \mathrm{H}), 7.23(\mathrm{~d}, J=8.0$ $\mathrm{Hz}, 2 \mathrm{H}), 6.81(\mathrm{~d}, J=9 \mathrm{~Hz}, 2 \mathrm{H}), 6.69(\mathrm{~d}, J=8.5 \mathrm{~Hz}, 2 \mathrm{H}), 3.69(\mathrm{~s}, 3 \mathrm{H}), 2.37(\mathrm{~s}, 3 \mathrm{H}) \mathrm{ppm} .{ }^{13} \mathrm{C}\left\{{ }^{1} \mathrm{H}\right\} \mathrm{NMR}$ (125 MHz, $\left.\mathrm{CDCl}_{3}\right): \delta$ 158.2, 145.3, 143.1, 132.3, 129.7, 128.6, 123.4, 114.5, 55.6, 21.7 ppm. HRMS (ESI-Orbitrap) m/z: (M) ${ }^{+}$calcd for $\mathrm{C}_{14} \mathrm{H}_{14} \mathrm{O}_{4} \mathrm{~S} 278.0613$, found 278.0616 . 


\section{References}

1. (a) E. T. Pelkey and G. W. Gribble, Synthesis, 1999, 1117. (b) Y. Lu, Y. Li, R. Zhang, K. Jin and C. Duan, Tetrahedron, 2013, 69, 9422.

2. Q. Cheng, H. - J. Zhang, W. - J. Yue and S. - L. You, Chem, 2017, 3, 428.

3. H. Kaida, T. Satoh, K. Hirano and M. Miura, Chem. Lett., 2015, 44, 1125.

4. D. Chen, D. Yuan, C. Zhang, H. Wu, J. Zhang, B. Li and X. Zhu, J. Org. Chem., 2017, 82, 10920.

5. K. Saito, P. K. Chikkade, M. Kanai and Y. Kuninobu, Chem. Eur. J., 2015, 21, 8365.

6. H. Huang, Y. Wu, W. Zhang, C. Feng, B. - Q. Wang, W. - F. Cai, P. Hu, K. - Q. Zhao and S. - K. Xiang, J. Org. Chem., 2017, 82, 3094. 


\section{7. ${ }^{1} \mathrm{H}$ NMR $\&{ }^{13} \mathrm{C}$ NMR Spectra}

${ }^{1} \mathrm{H}$ NMR $\left(500 \mathrm{MHz}, \mathrm{CDCl}_{3}\right) \&{ }^{13} \mathrm{C}\left\{{ }^{1} \mathrm{H}\right\}(125 \mathrm{MHz}, \mathrm{CDCl}$ ) Spectra of 3a

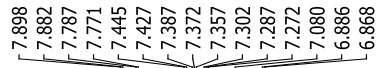

$\stackrel{\text { ळे }}{i}$
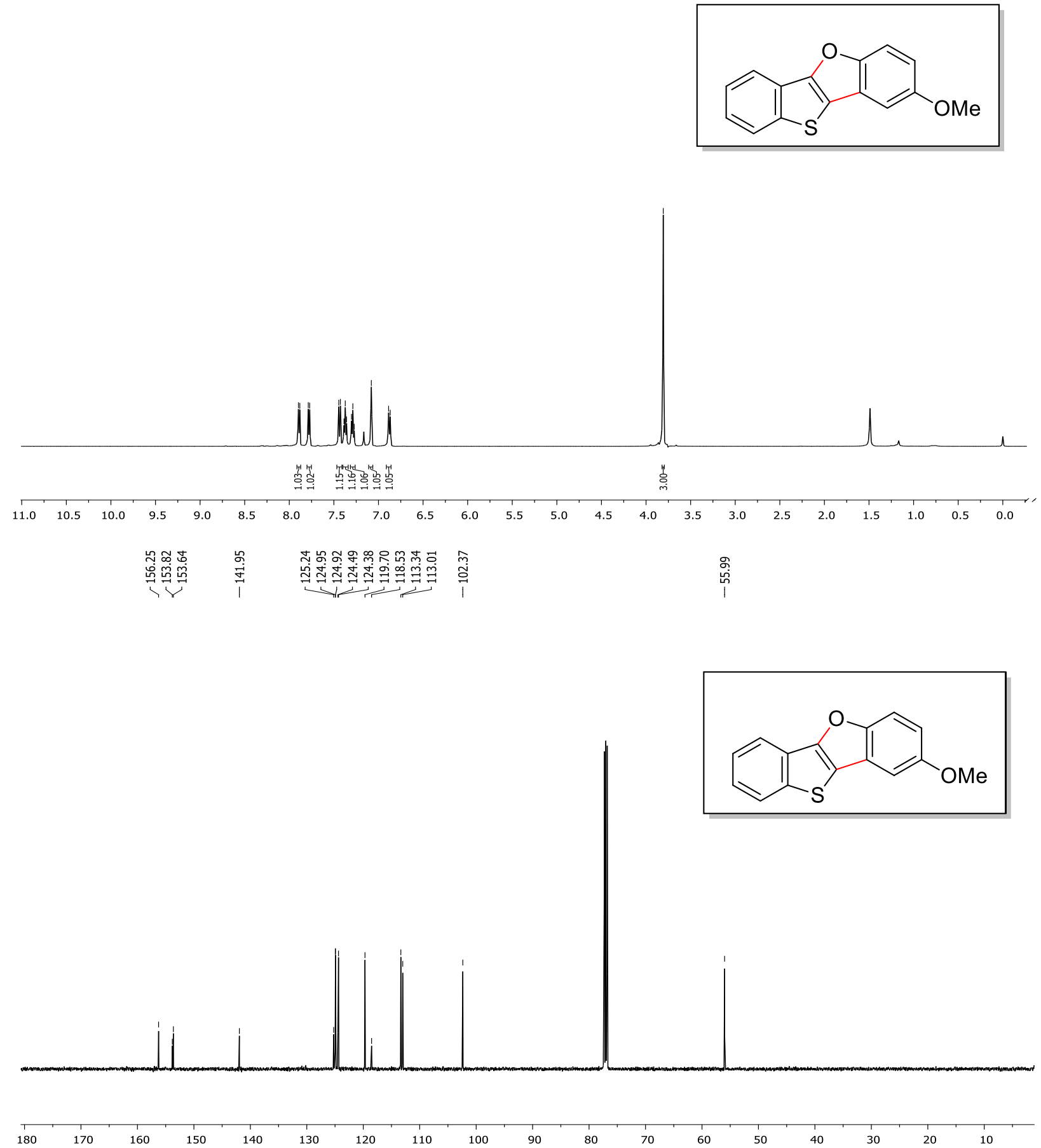
${ }^{1} \mathrm{H}$ NMR $\left(500 \mathrm{MHz}, \mathrm{CDCl}_{3}\right) \&{ }^{13} \mathrm{C}\left\{{ }^{1} \mathrm{H}\right\}\left(125 \mathrm{MHz}, \mathrm{CDCl}_{3}\right)$ Spectra of $\mathbf{3 b}$

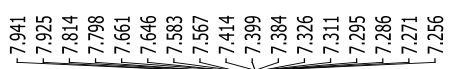
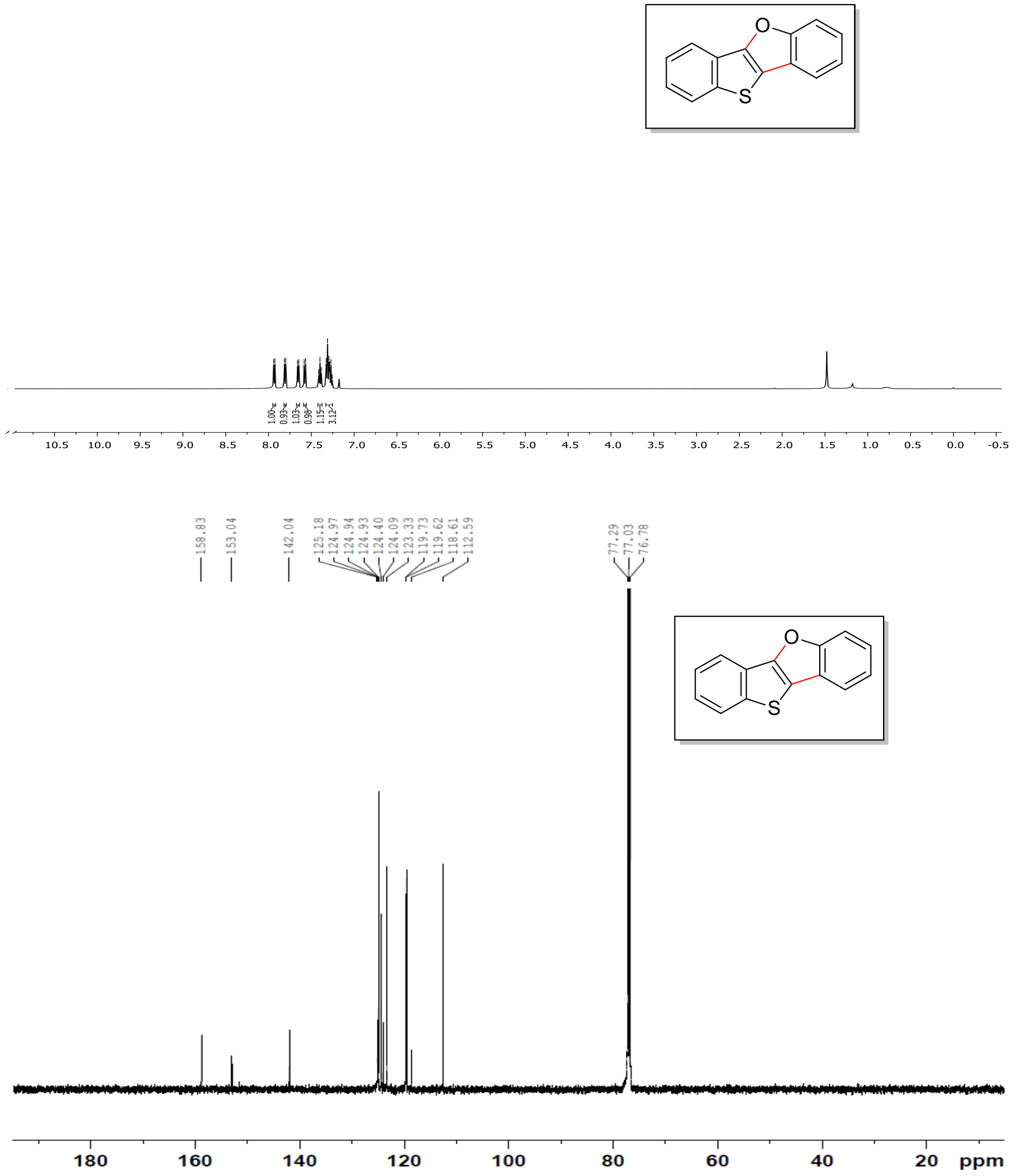
${ }^{1} \mathrm{H}$ NMR $\left(500 \mathrm{MHz}, \mathrm{CDCl}_{3}\right) \&{ }^{13} \mathrm{C}\left\{{ }^{1} \mathrm{H}\right\}\left(125 \mathrm{MHz}, \mathrm{CDCl}_{3}\right)$ Spectra of 3c

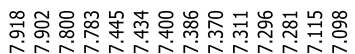
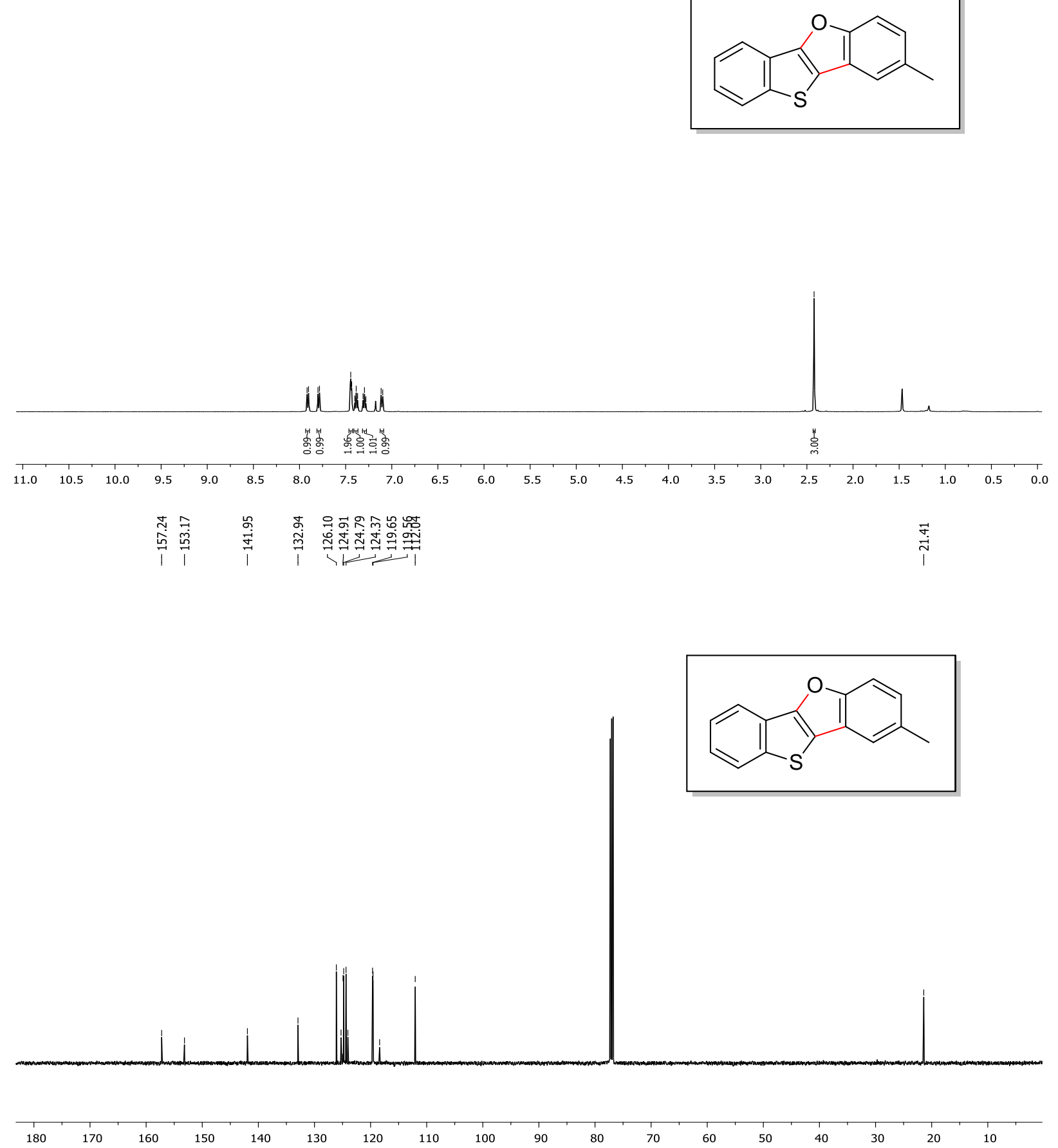
${ }^{1} \mathrm{H}$ NMR $\left(500 \mathrm{MHz}, \mathrm{CDCl}_{3}\right) \&{ }^{13} \mathrm{C}\left\{{ }^{1} \mathrm{H}\right\}\left(125 \mathrm{MHz}, \mathrm{CDCl}_{3}\right)$ Spectra of $\mathbf{3 d}$

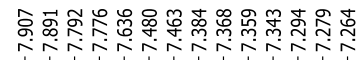
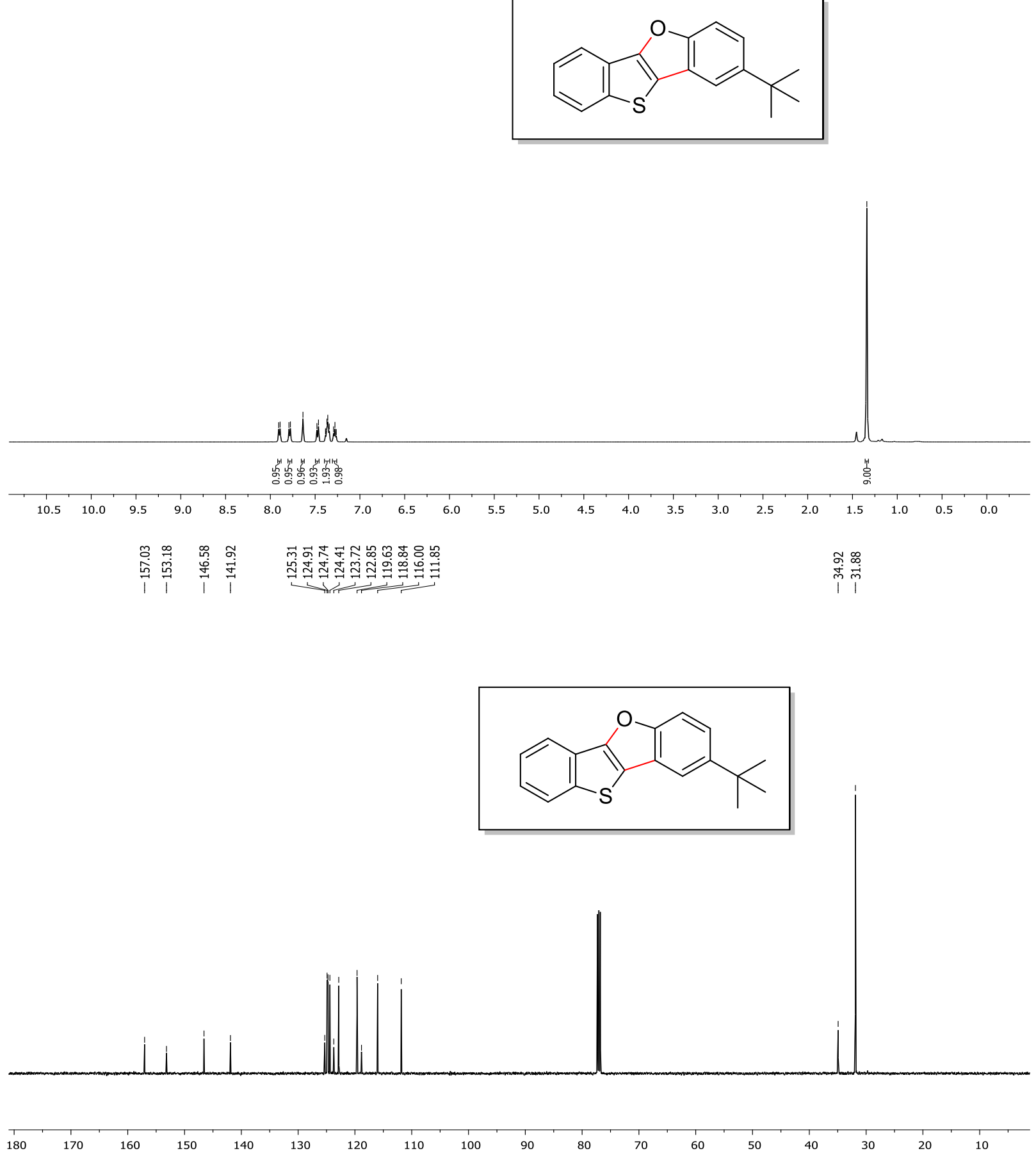
${ }^{1} \mathrm{H}$ NMR $\left(500 \mathrm{MHz}, \mathrm{CDCl}_{3}\right) \&{ }^{13} \mathrm{C}\left\{{ }^{1} \mathrm{H}\right\}\left(125 \mathrm{MHz}, \mathrm{CDCl}_{3}\right)$ Spectra of $\mathbf{3 e}$

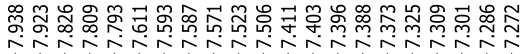
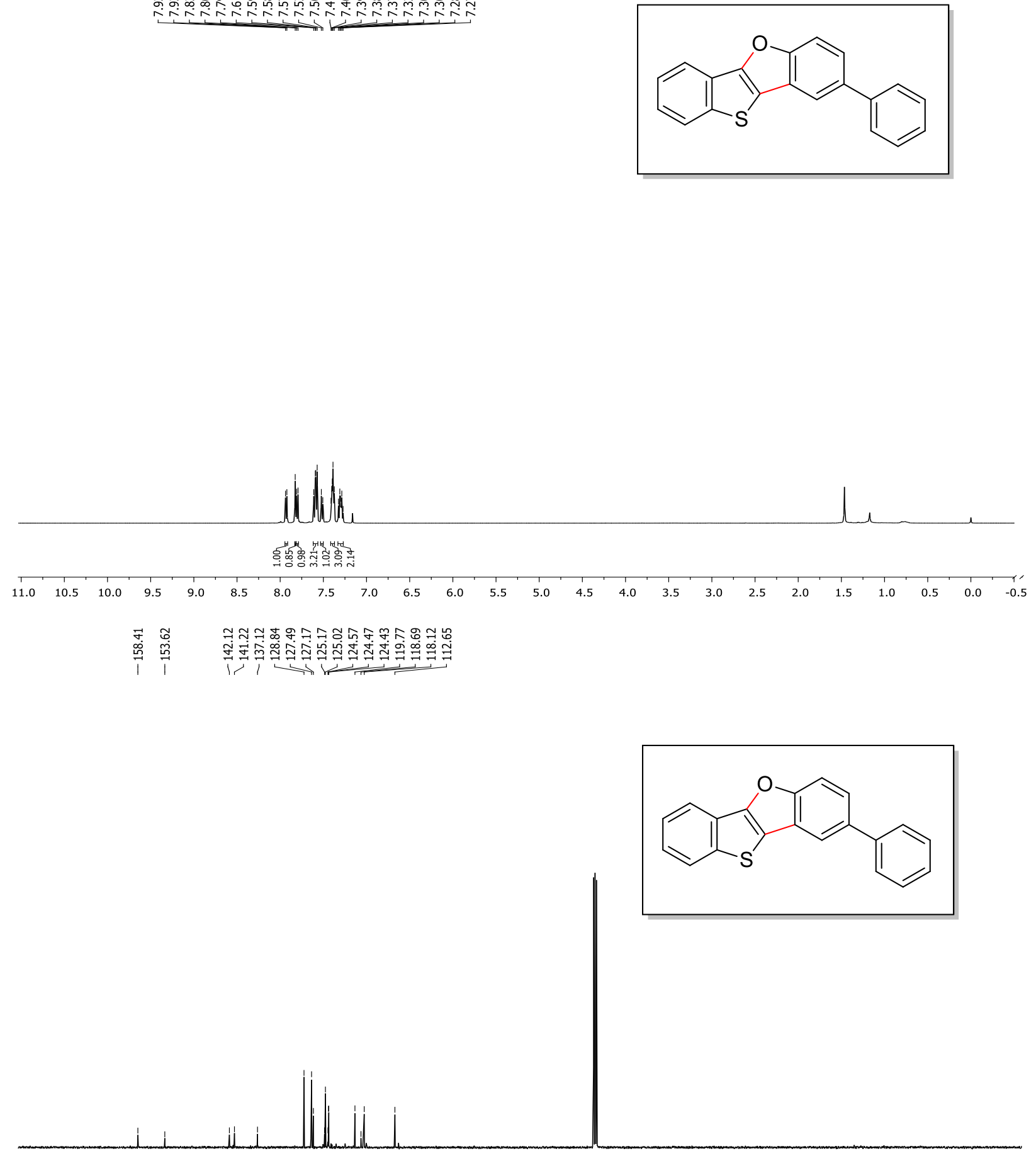
${ }^{1} \mathrm{H}$ NMR $\left(500 \mathrm{MHz}, \mathrm{CDCl}_{3}\right) \&{ }^{13} \mathrm{C}\left\{{ }^{1} \mathrm{H}\right\}\left(125 \mathrm{MHz}, \mathrm{CDCl}_{3}\right)$ Spectra of $\mathbf{3 g}$

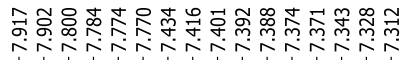
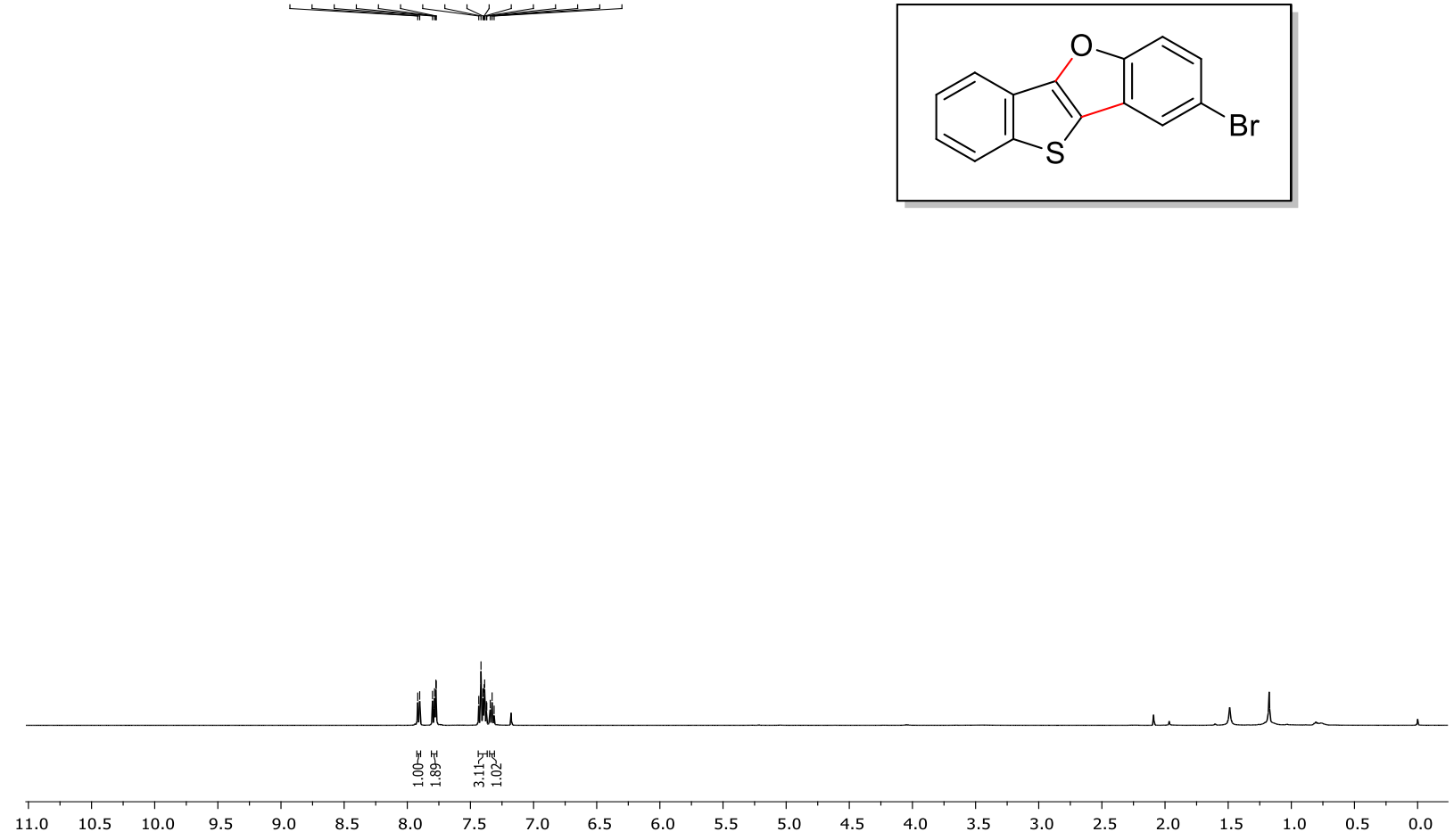

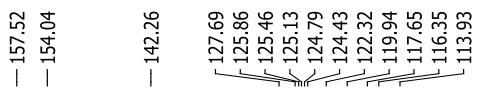
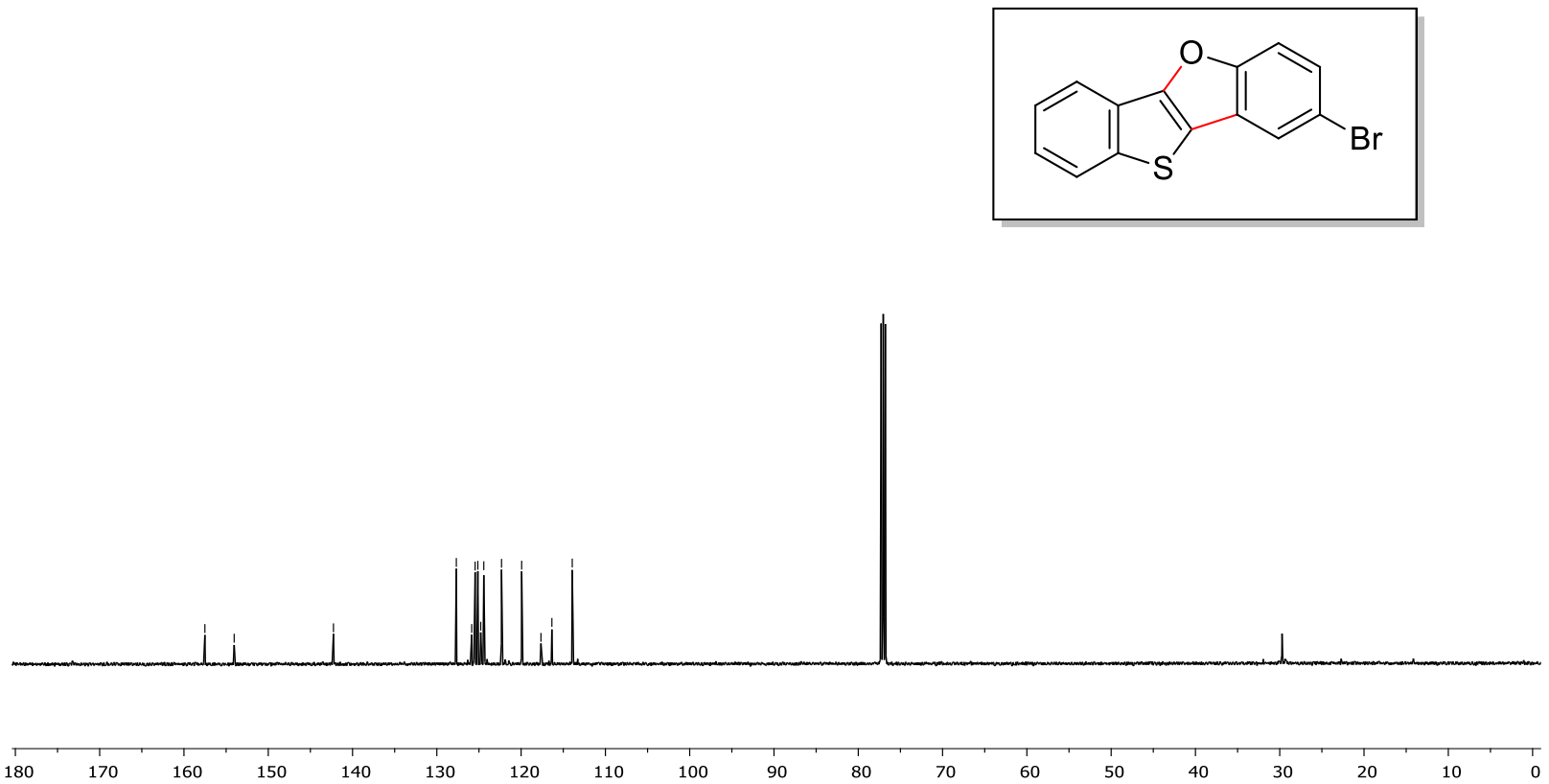
${ }^{1} \mathrm{H}$ NMR $\left(500 \mathrm{MHz}, \mathrm{CDCl}_{3}\right) \&{ }^{13} \mathrm{C}\left\{{ }^{1} \mathrm{H}\right\}\left(125 \mathrm{MHz}, \mathrm{CDCl}_{3}\right)$ Spectra of $\mathbf{3 h}$

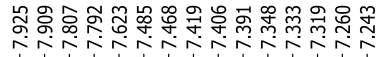
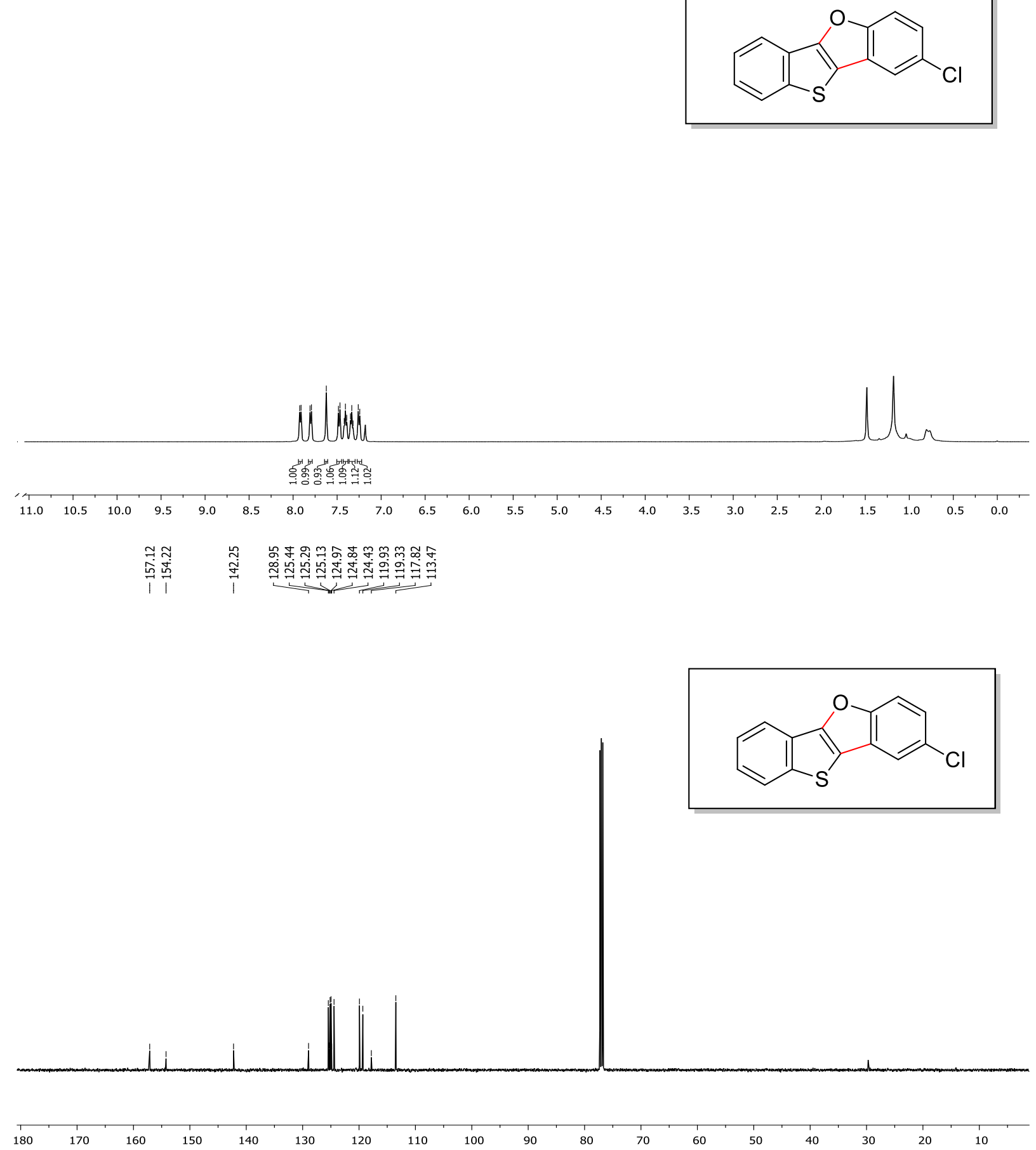
${ }^{1} \mathrm{H}$ NMR $\left(500 \mathrm{MHz}, \mathrm{CDCl}_{3}\right) \&{ }^{13} \mathrm{C}\left\{{ }^{1} \mathrm{H}\right\}\left(125 \mathrm{MHz}, \mathrm{CDCl}_{3}\right)$ Spectra of $\mathbf{3 i}$

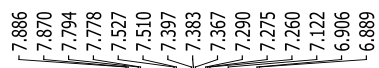

$\underset{\substack{m \\ i}}{\stackrel{m}{j}}$
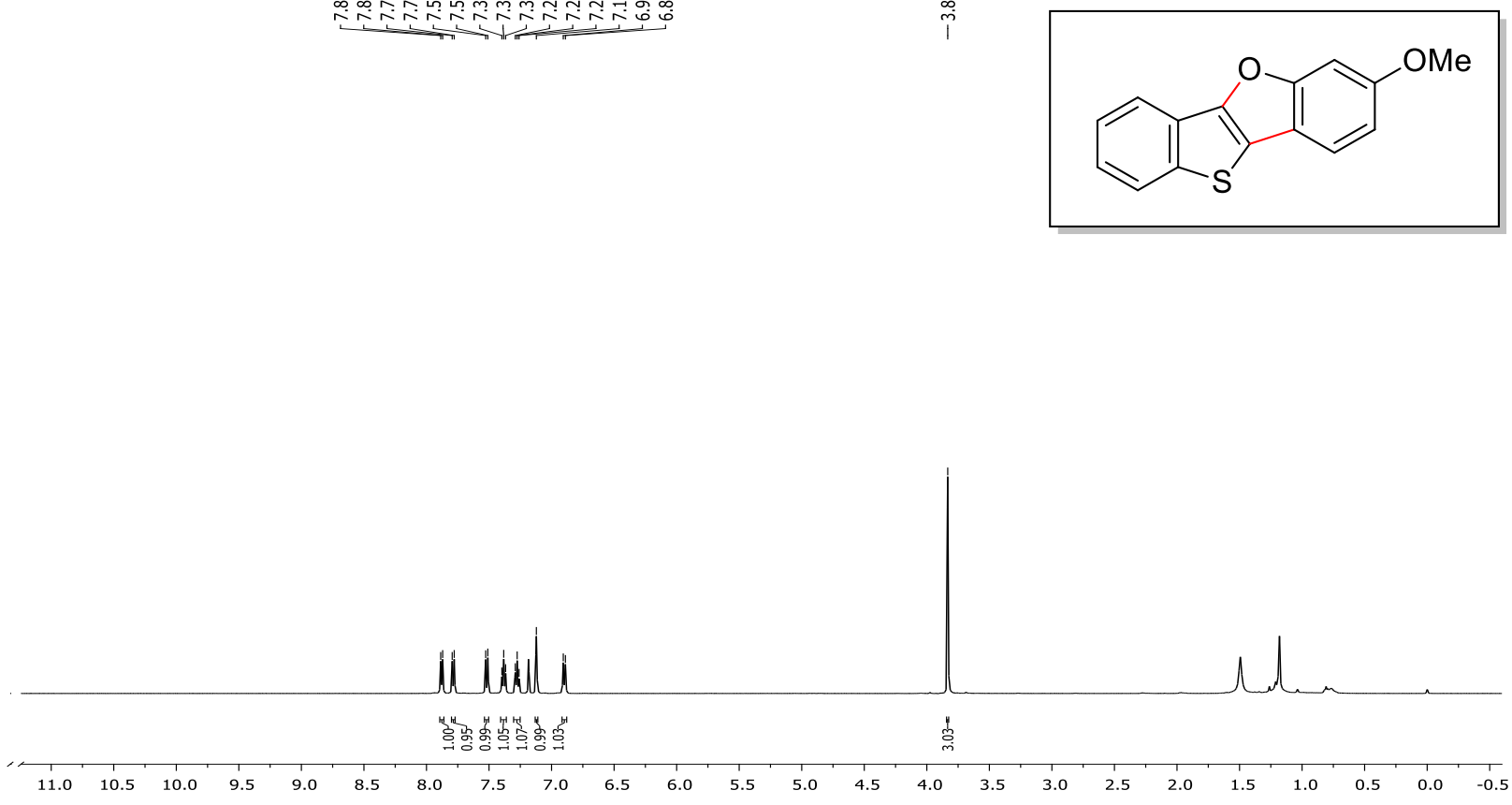

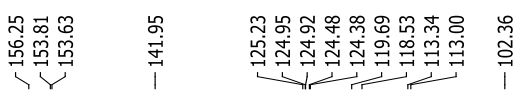

옹

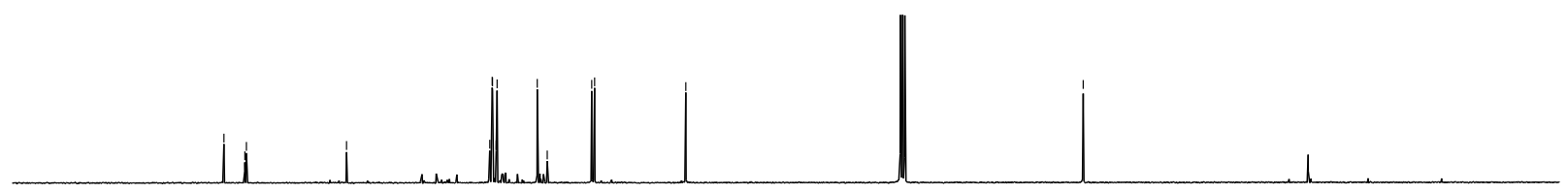

180

$170 \quad 170$
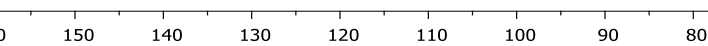
${ }^{1} \mathrm{H}$ NMR $\left(500 \mathrm{MHz}, \mathrm{CDCl}_{3}\right) \&{ }^{13} \mathrm{C}\left\{{ }^{1} \mathrm{H}\right\}\left(125 \mathrm{MHz}, \mathrm{CDCl}_{3}\right)$ Spectra of $\mathbf{3 j}$
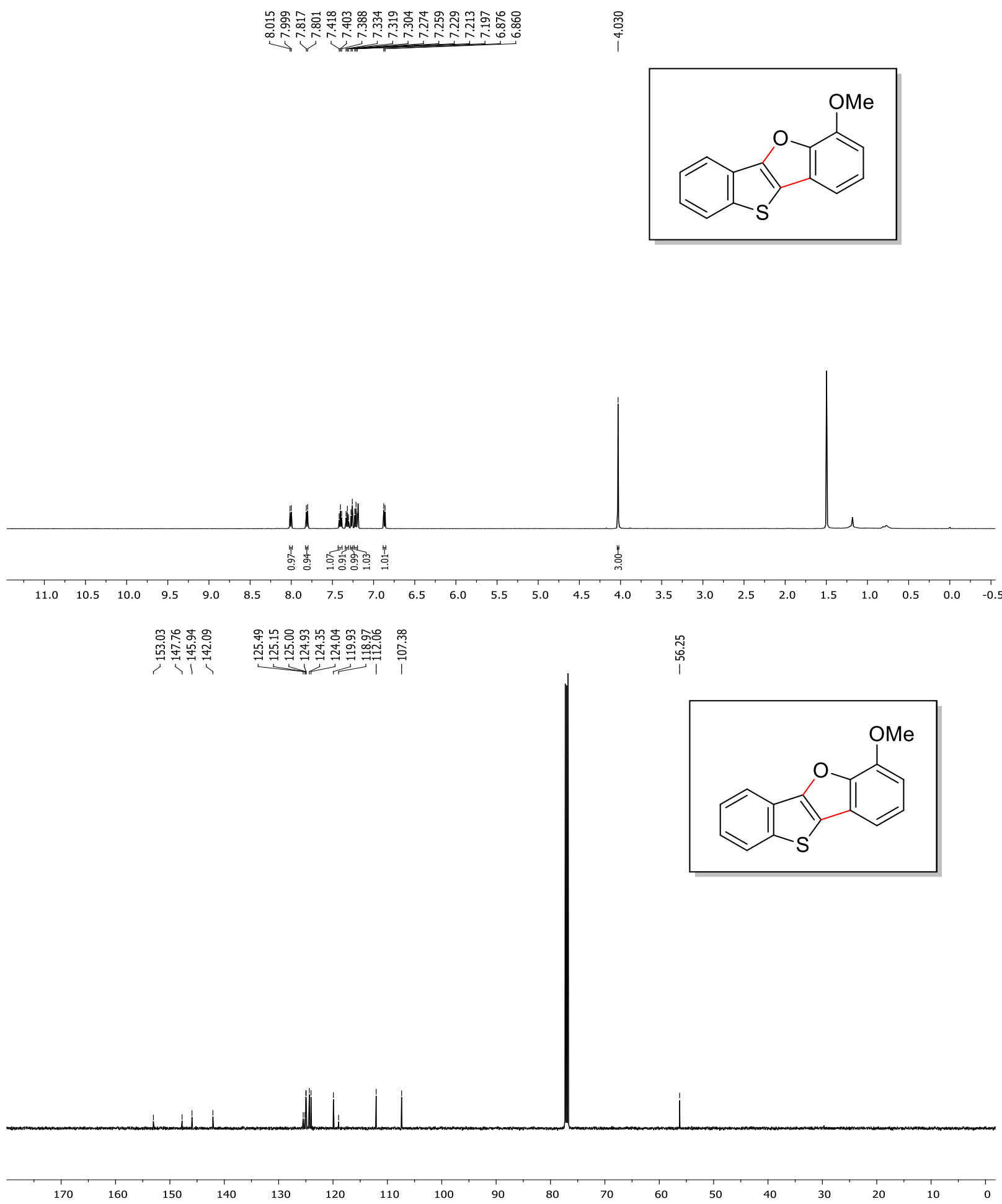
${ }^{1} \mathrm{H}$ NMR $\left(500 \mathrm{MHz}, \mathrm{CDCl}_{3}\right) \&{ }^{13} \mathrm{C}\left\{{ }^{1} \mathrm{H}\right\}\left(125 \mathrm{MHz}, \mathrm{CDCl}_{3}\right)$ Spectra of 3k

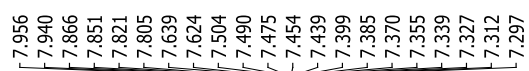
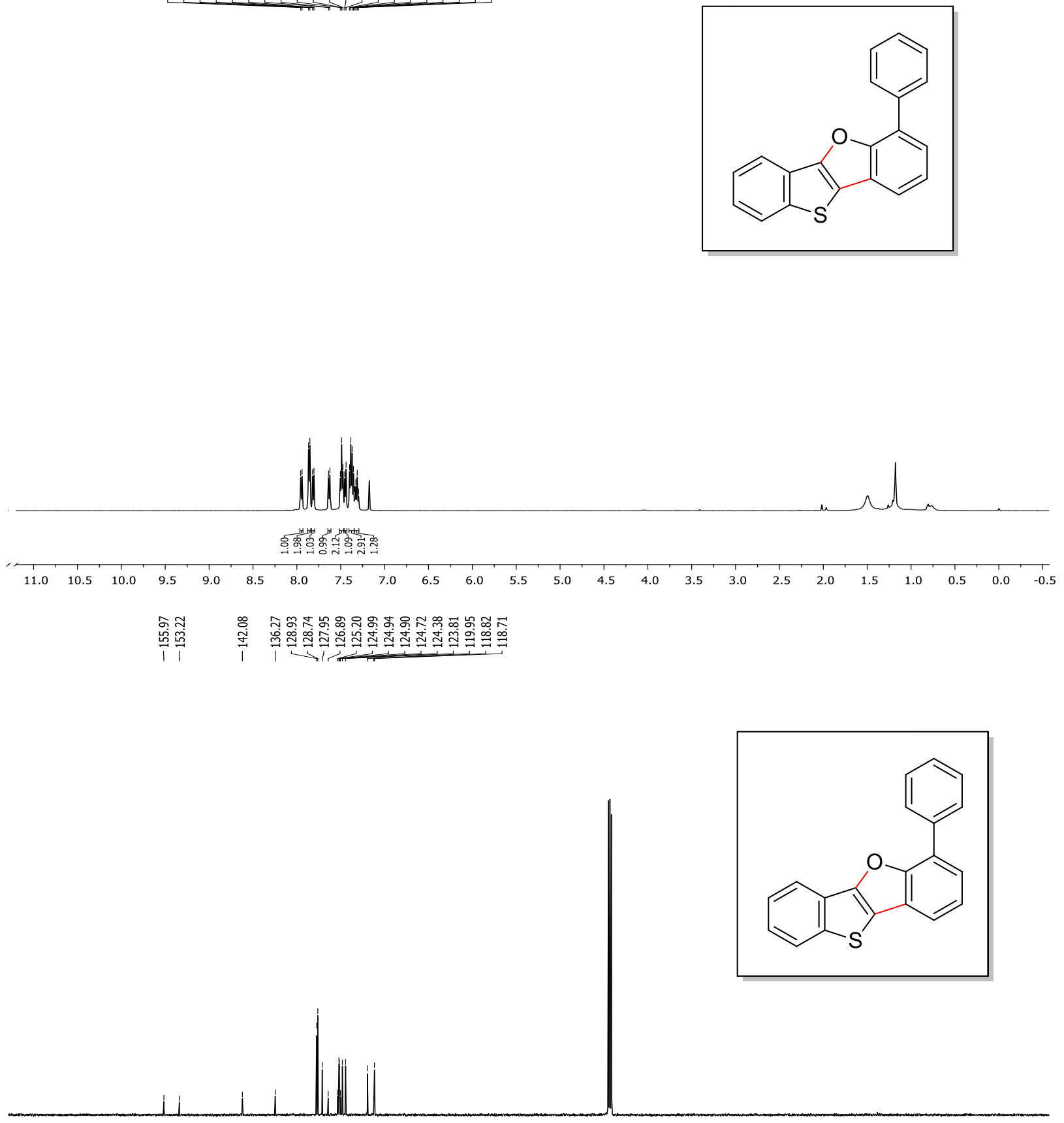

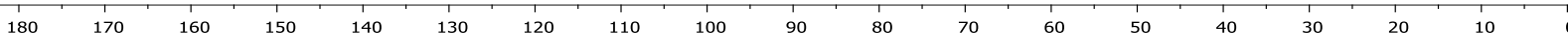


${ }^{1} \mathrm{H}$ NMR $\left(500 \mathrm{MHz}, \mathrm{CDCl}_{3}\right) \&{ }^{13} \mathrm{C}\left\{{ }^{1} \mathrm{H}\right\}\left(125 \mathrm{MHz}, \mathrm{CDCl}_{3}\right)$ Spectra of $3 \mathrm{~m}$
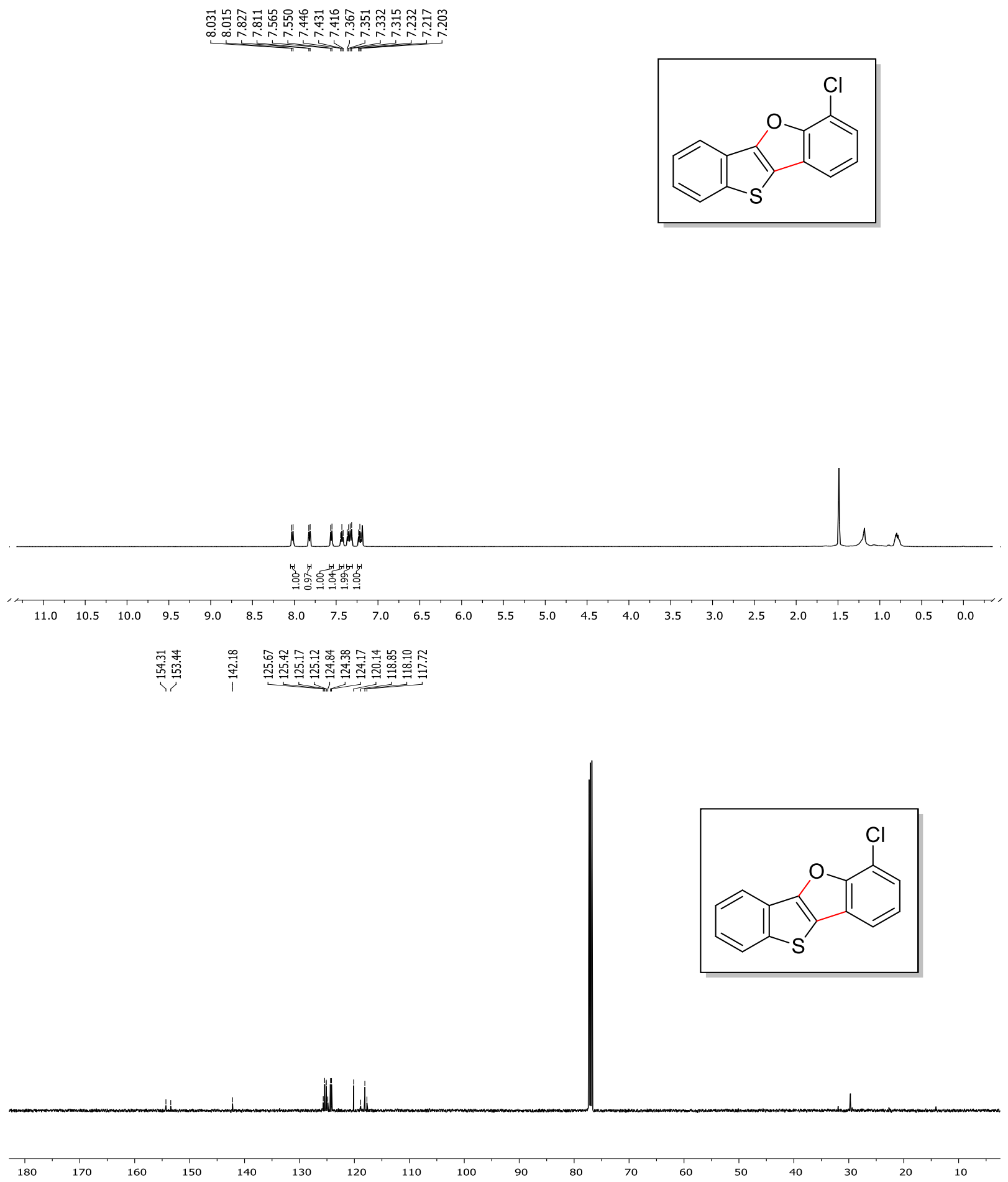
${ }^{1} \mathrm{H}$ NMR $\left(500 \mathrm{MHz}, \mathrm{CDCl}_{3}\right) \&{ }^{13} \mathrm{C}\left\{{ }^{1} \mathrm{H}\right\}\left(125 \mathrm{MHz}, \mathrm{CDCl}_{3}\right)$ Spectra of $\mathbf{3 n}$

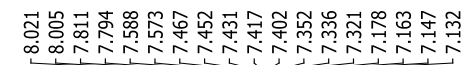
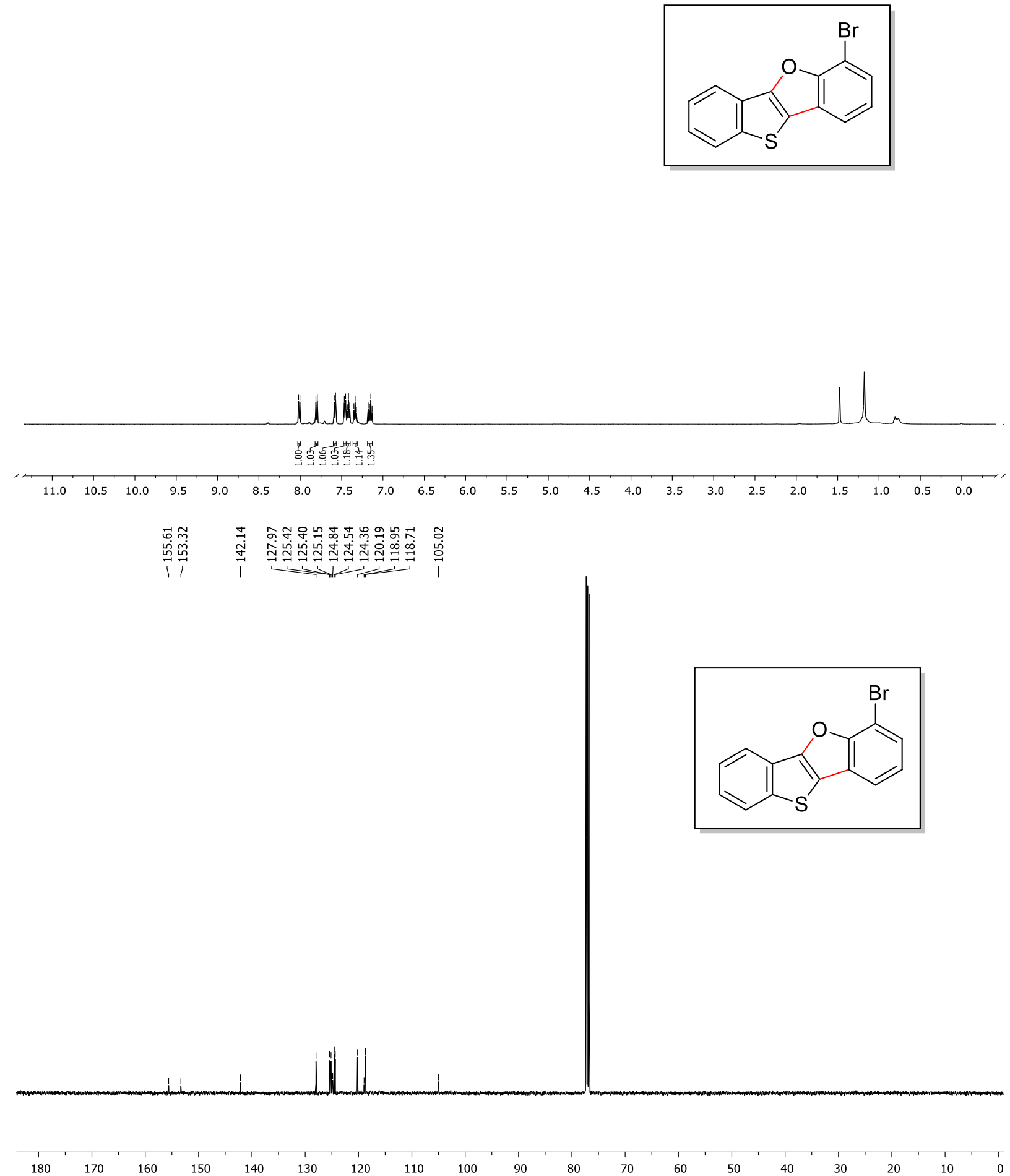
${ }^{1} \mathrm{H}$ NMR $\left(500 \mathrm{MHz}, \mathrm{CDCl}_{3}\right) \&{ }^{13} \mathrm{C}\left\{{ }^{1} \mathrm{H}\right\}\left(125 \mathrm{MHz}, \mathrm{CDCl}_{3}\right)$ Spectra of 30

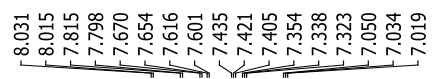

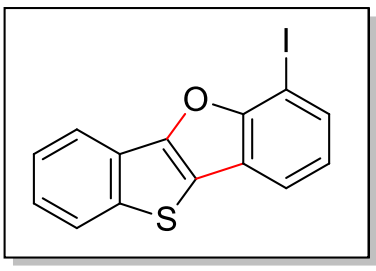

Willibie
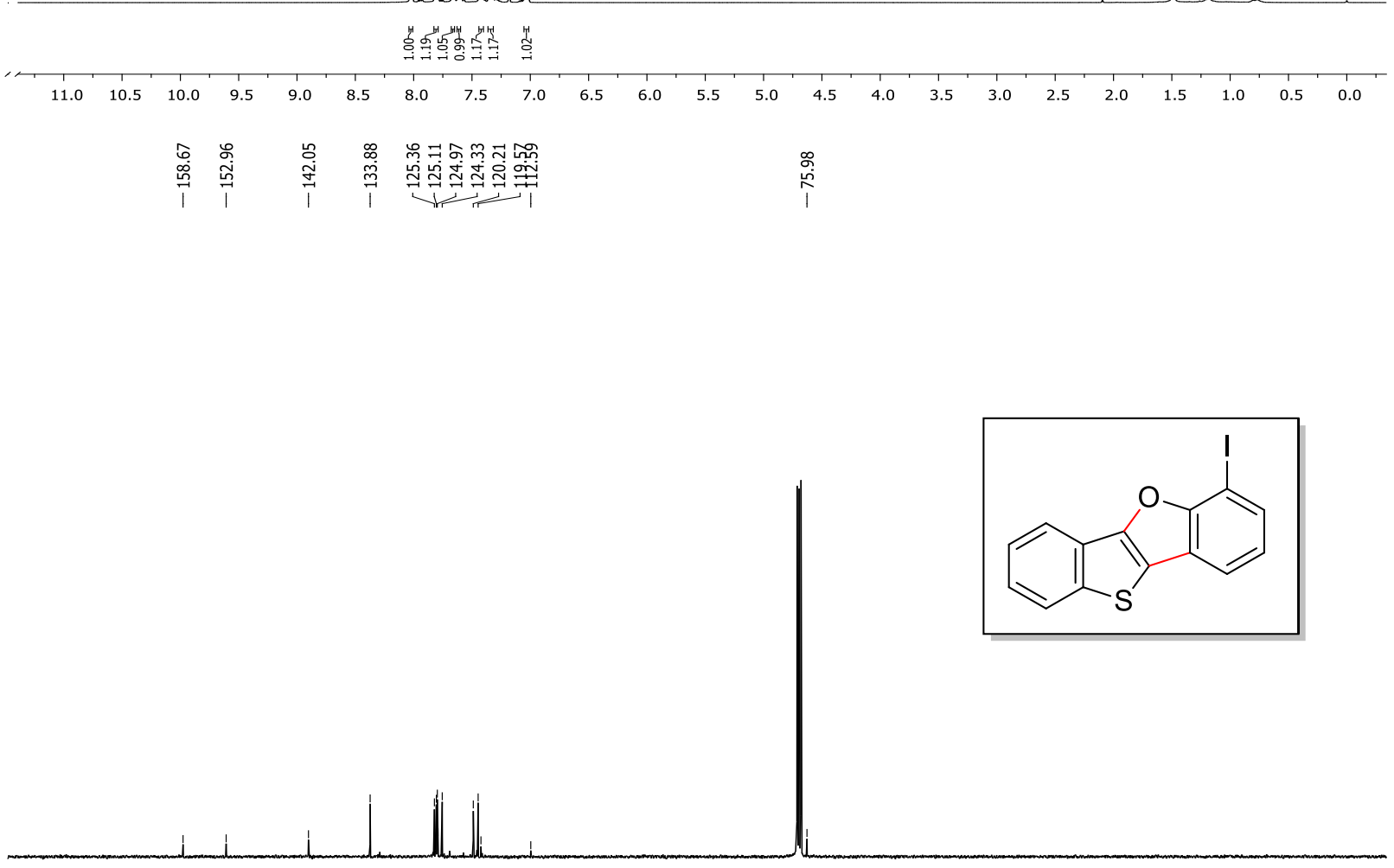

180

180

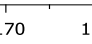

160

130

120

110

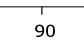

80

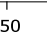

$30 \quad 20 \quad 10 \quad 5$ 
${ }^{1} \mathrm{H}$ NMR $\left(500 \mathrm{MHz}, \mathrm{CDCl}_{3}\right) \&{ }^{13} \mathrm{C}\left\{{ }^{1} \mathrm{H}\right\}\left(125 \mathrm{MHz}, \mathrm{CDCl}_{3}\right)$ Spectra of $3 p$

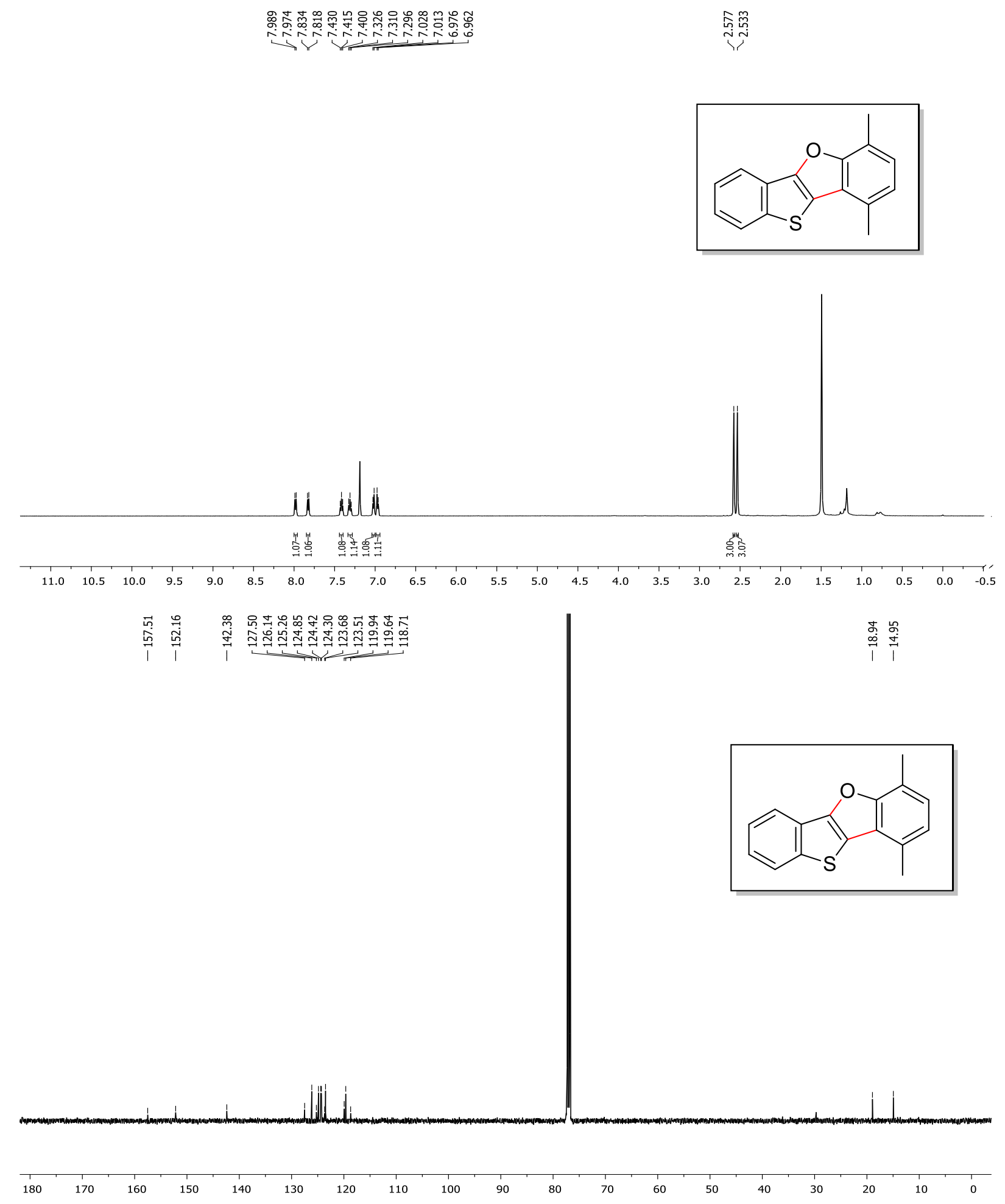


${ }^{1} \mathrm{H}$ NMR $\left(500 \mathrm{MHz}, \mathrm{CDCl}_{3}\right) \&{ }^{13} \mathrm{C}\left\{{ }^{1} \mathrm{H}\right\}\left(125 \mathrm{MHz}, \mathrm{CDCl}_{3}\right)$ Spectra of $\mathbf{3 q}$

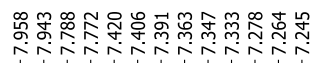

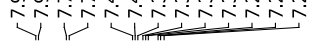

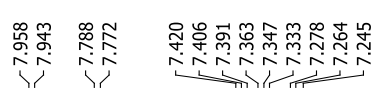
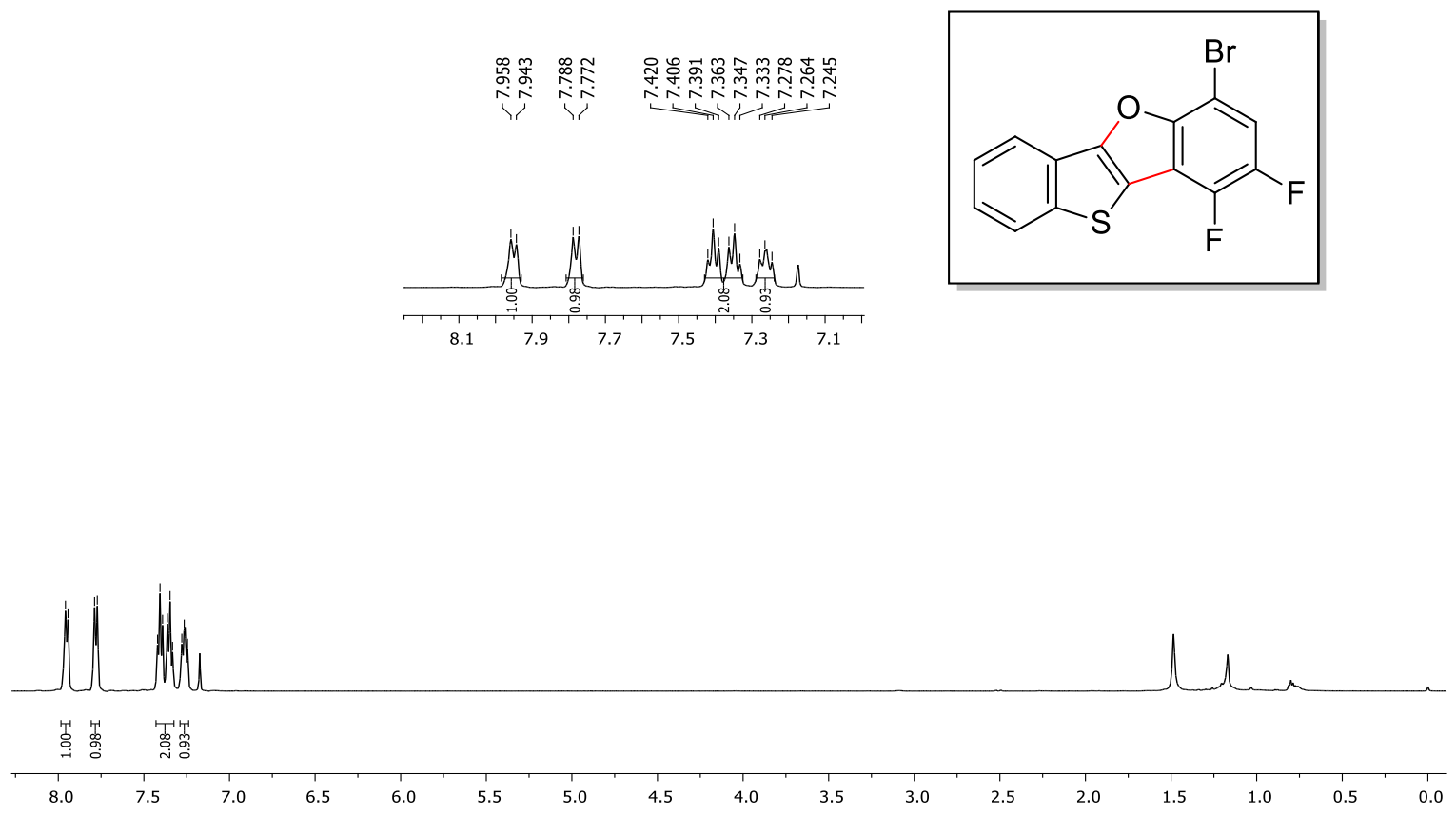

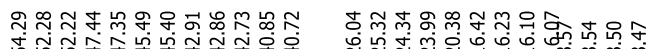

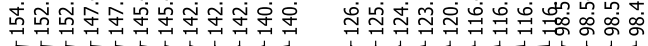

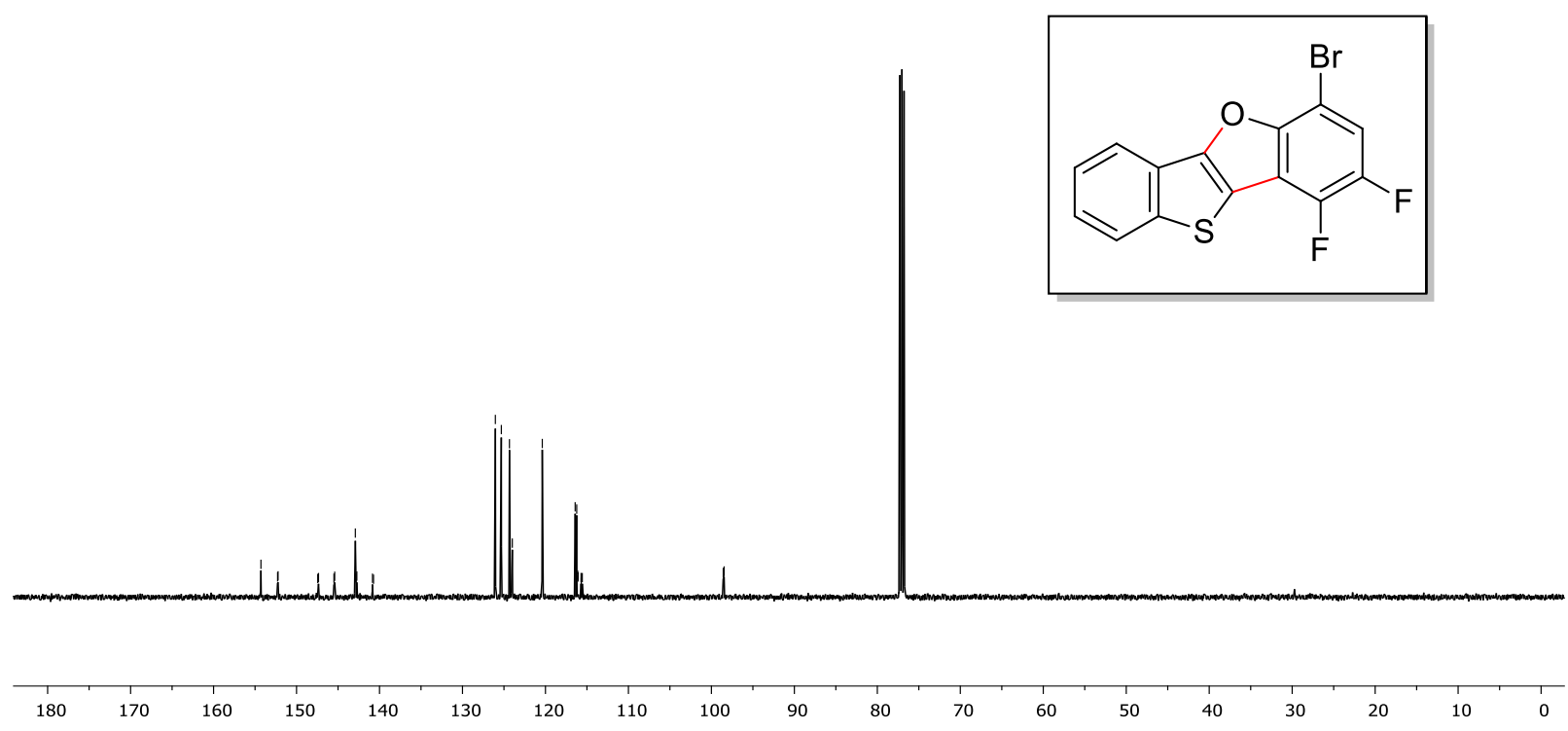


${ }^{1} \mathrm{H}$ NMR $\left(500 \mathrm{MHz}, \mathrm{CDCl}_{3}\right) \&{ }^{13} \mathrm{C}\left\{{ }^{1} \mathrm{H}\right\}\left(125 \mathrm{MHz}, \mathrm{CDCl}_{3}\right)$ Spectra of $3 \mathbf{r}$

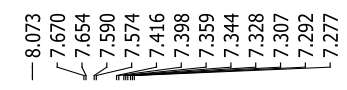
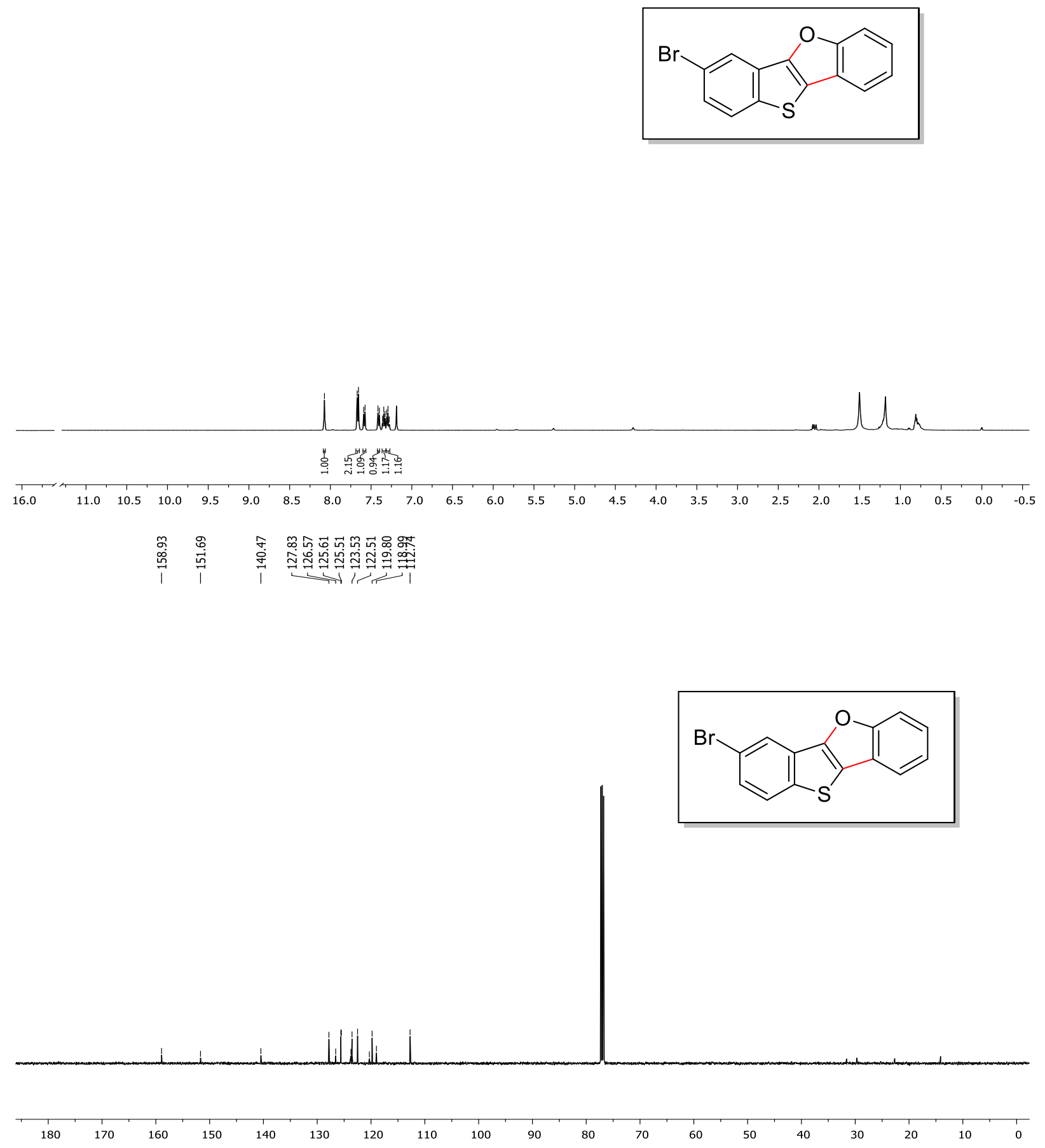
${ }^{1} \mathrm{H}$ NMR $\left(500 \mathrm{MHz}, \mathrm{CDCl}_{3}\right) \&{ }^{13} \mathrm{C}\left\{{ }^{1} \mathrm{H}\right\}\left(125 \mathrm{MHz}, \mathrm{CDCl}_{3}\right)$ Spectra of 3s

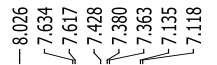

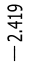
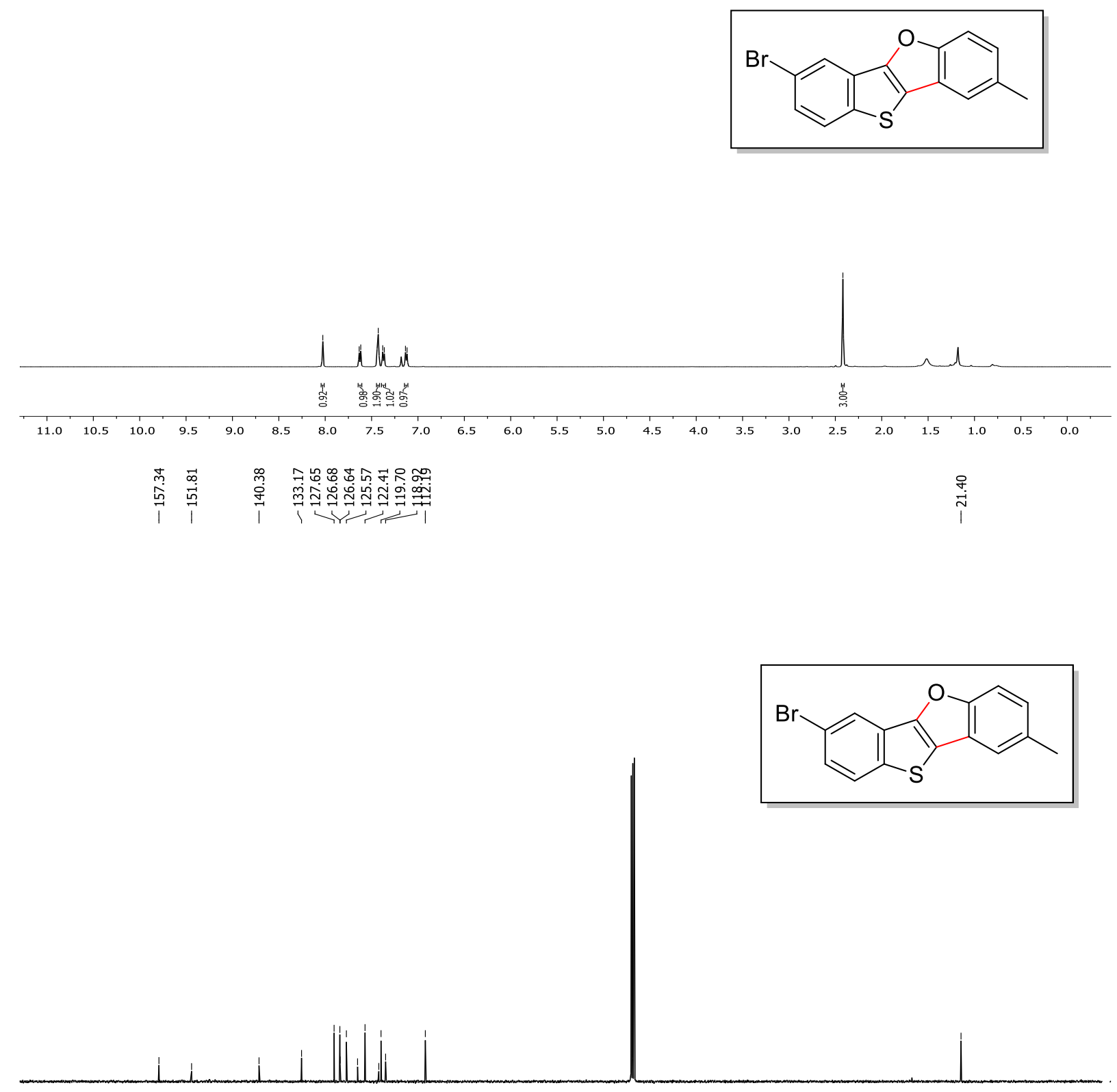

180

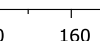

120

90 
${ }^{1} \mathrm{H}$ NMR $\left(500 \mathrm{MHz}, \mathrm{CDCl}_{3}\right) \&{ }^{13} \mathrm{C}\left\{{ }^{1} \mathrm{H}\right\}\left(125 \mathrm{MHz}, \mathrm{CDCl}_{3}\right)$ Spectra of 3t

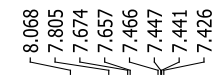
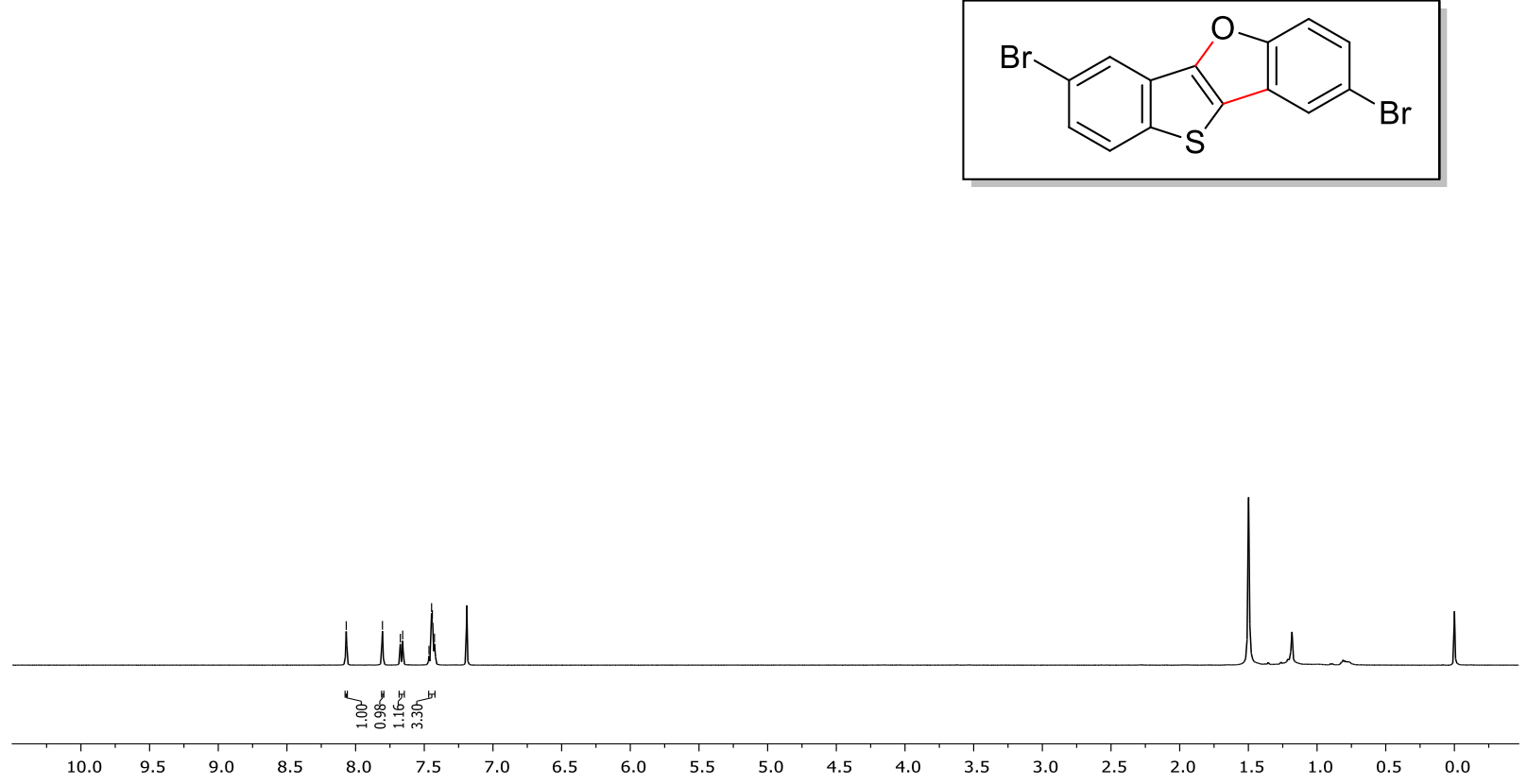

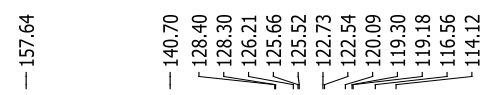
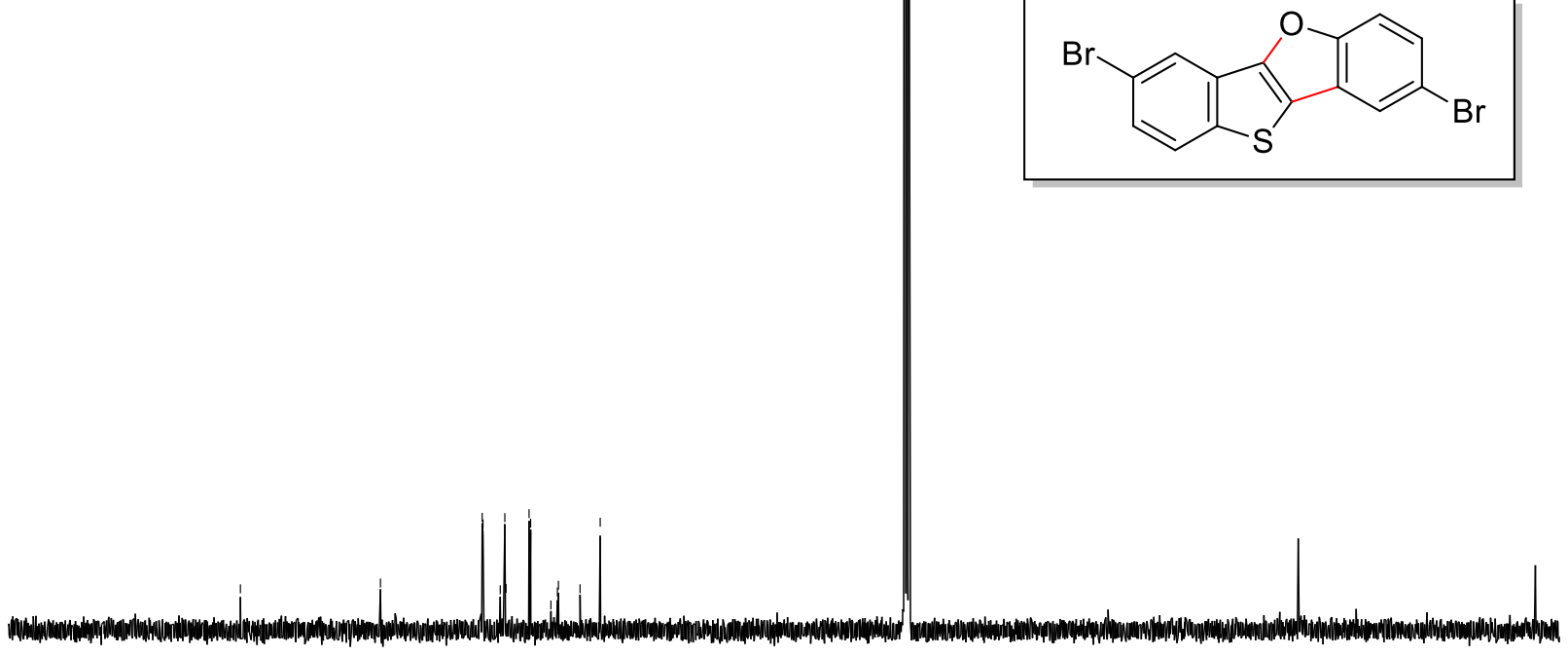
${ }^{1} \mathrm{H}$ NMR $\left(500 \mathrm{MHz}, \mathrm{CDCl}_{3}\right) \&{ }^{13} \mathrm{C}\left\{{ }^{1} \mathrm{H}\right\}\left(125 \mathrm{MHz}, \mathrm{CDCl}_{3}\right)$ Spectra of $3 u$

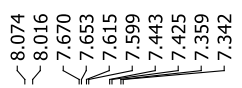
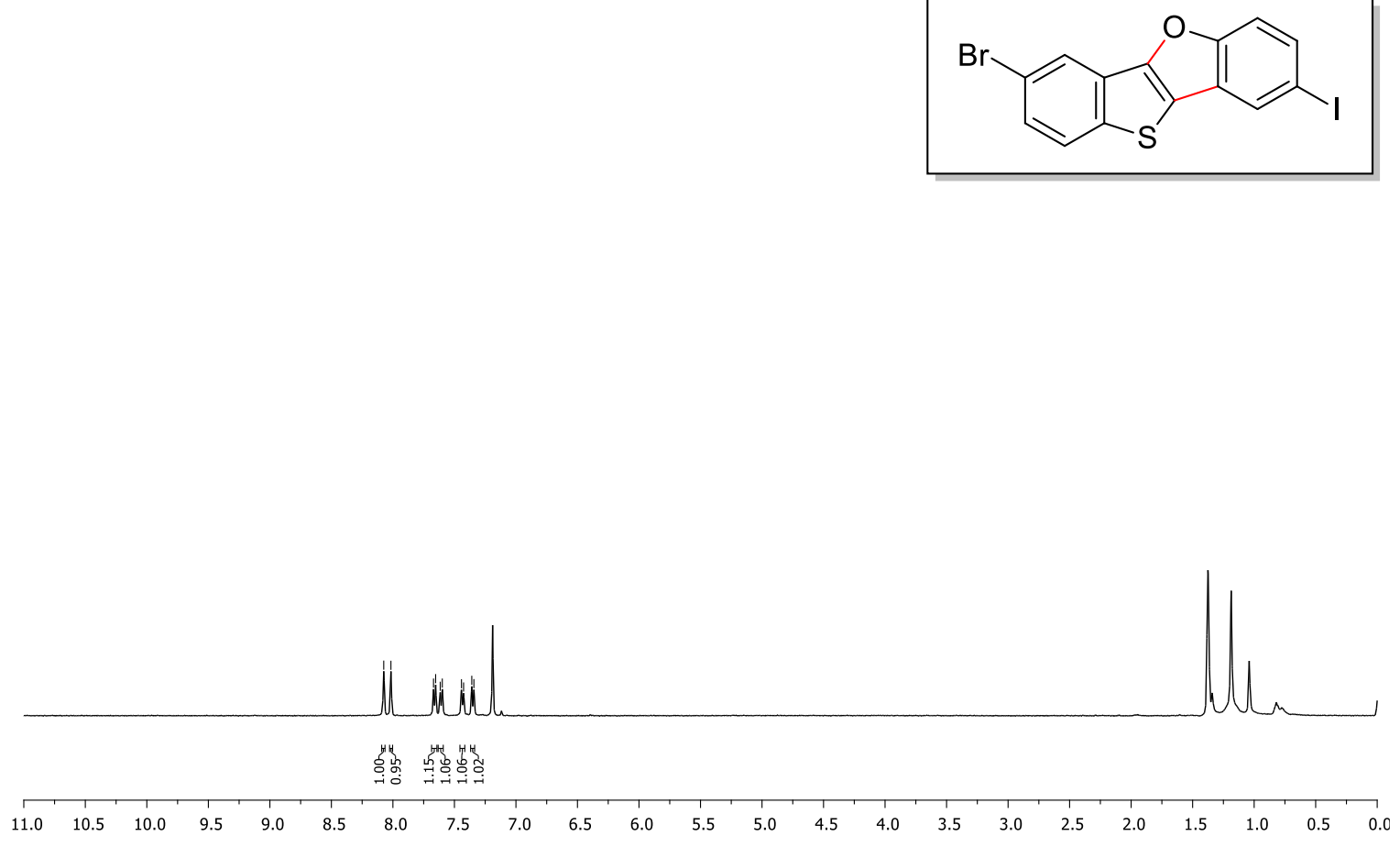

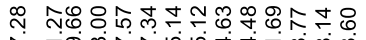

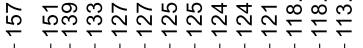

1 (1)

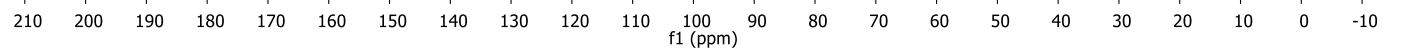


${ }^{1} \mathrm{H}$ NMR $\left(500 \mathrm{MHz}, \mathrm{CDCl}_{3}\right) \&{ }^{13} \mathrm{C}\left\{{ }^{1} \mathrm{H}\right\}\left(125 \mathrm{MHz}, \mathrm{CDCl}_{3}\right)$ Spectra of $3 \mathbf{v}$

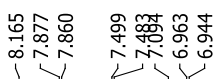

$\underset{\infty}{\infty}$
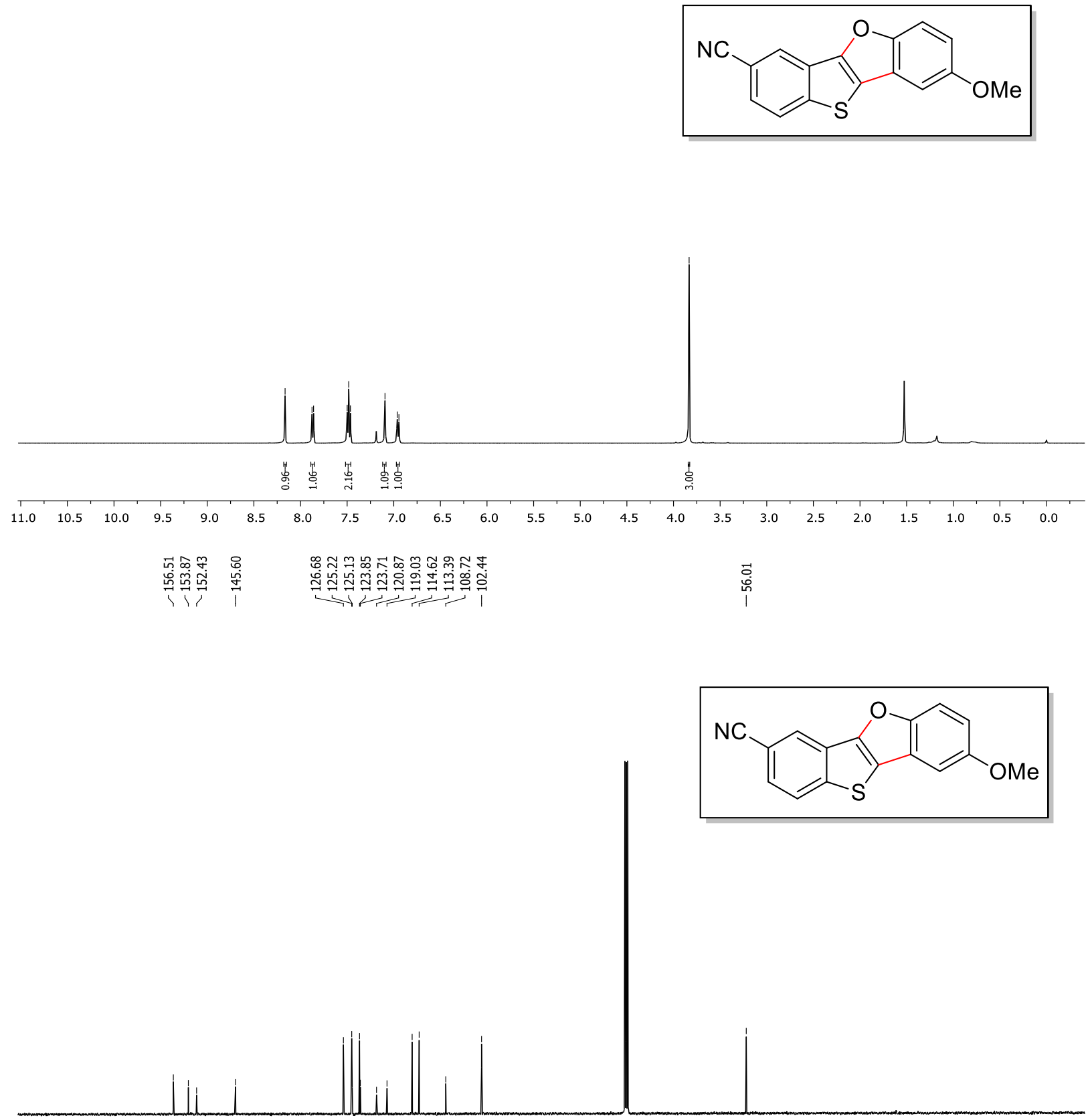
${ }^{1} \mathrm{H}$ NMR $\left(500 \mathrm{MHz}, \mathrm{CDCl}_{3}\right) \&{ }^{13} \mathrm{C}\left\{{ }^{1} \mathrm{H}\right\}\left(125 \mathrm{MHz}, \mathrm{CDCl}_{3}\right)$ Spectra of $\mathbf{3 w}$

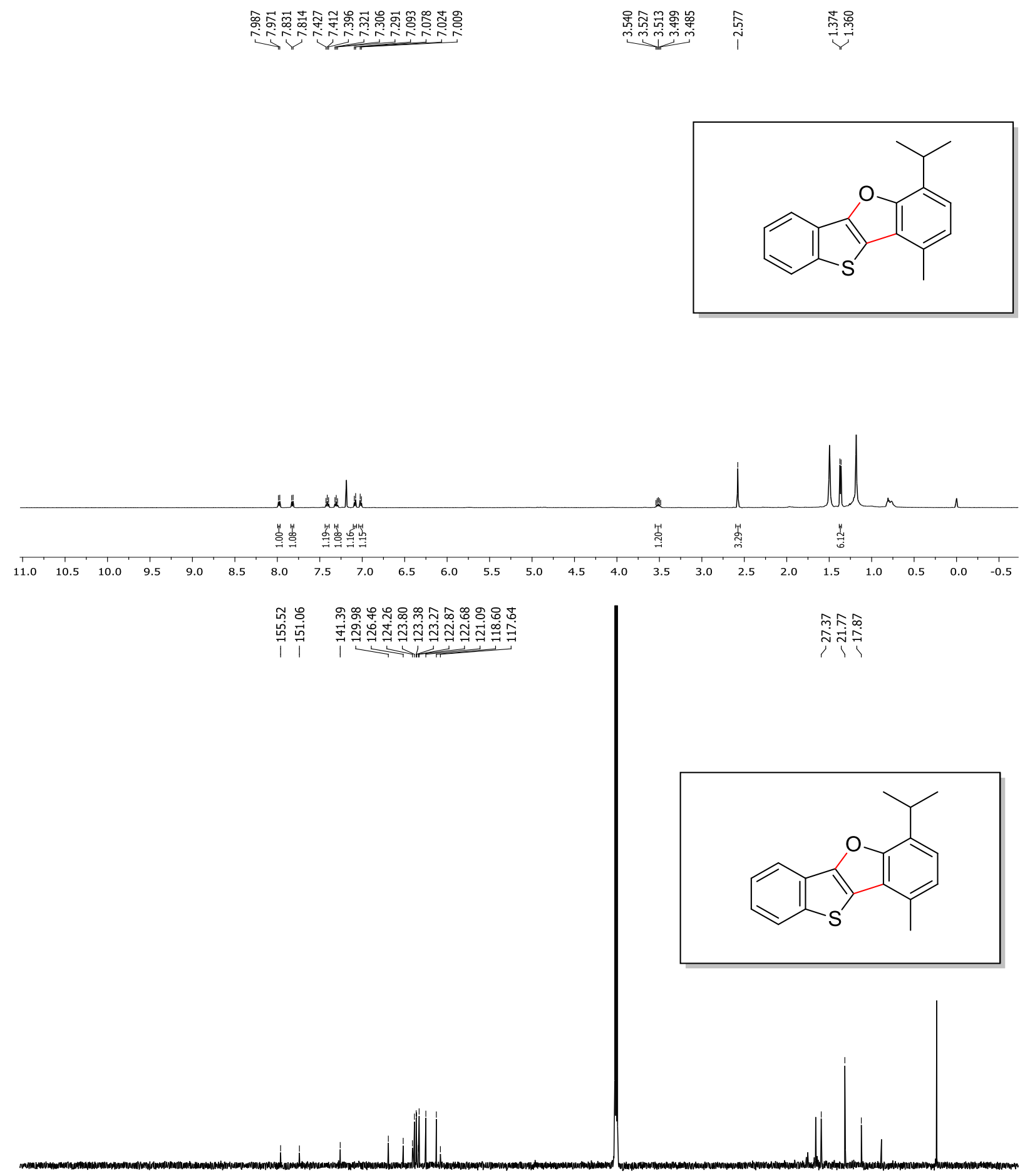

$\begin{array}{lllllllllllllllllllllllllllllll}210 & 200 & 190 & 180 & 170 & 160 & 150 & 140 & 130 & 120 & 110 & 100 & 90 & 80 & 70 & 60 & 50 & 40 & 30 & 20 & 10 & 0 & -10 & 10\end{array}$ 
${ }^{1} \mathrm{H}$ NMR $\left(500 \mathrm{MHz}, \mathrm{CDCl}_{3}\right) \&{ }^{13} \mathrm{C}\left\{{ }^{1} \mathrm{H}\right\}\left(125 \mathrm{MHz}, \mathrm{CDCl}_{3}\right)$ Spectra of $\mathbf{3 x}$

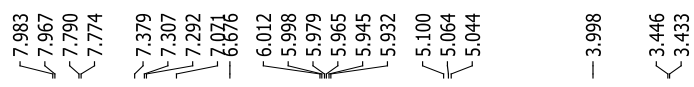
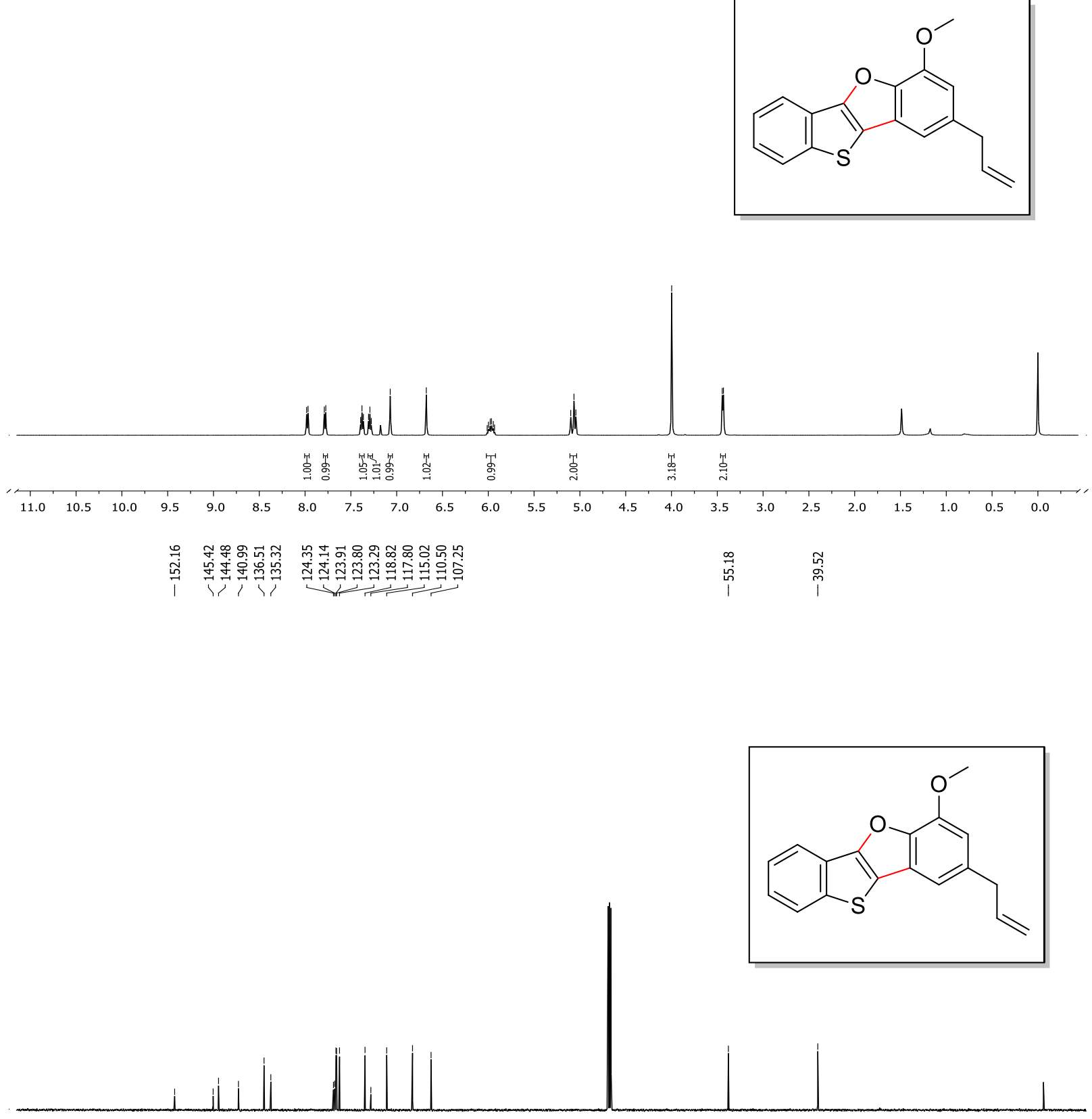
${ }^{1} \mathrm{H}$ NMR $\left(500 \mathrm{MHz}, \mathrm{CDCl}_{3}\right) \&{ }^{13} \mathrm{C}\left\{{ }^{1} \mathrm{H}\right\}\left(125 \mathrm{MHz}, \mathrm{CDCl}_{3}\right)$ Spectra of 3y

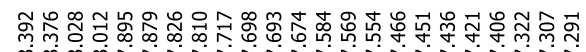
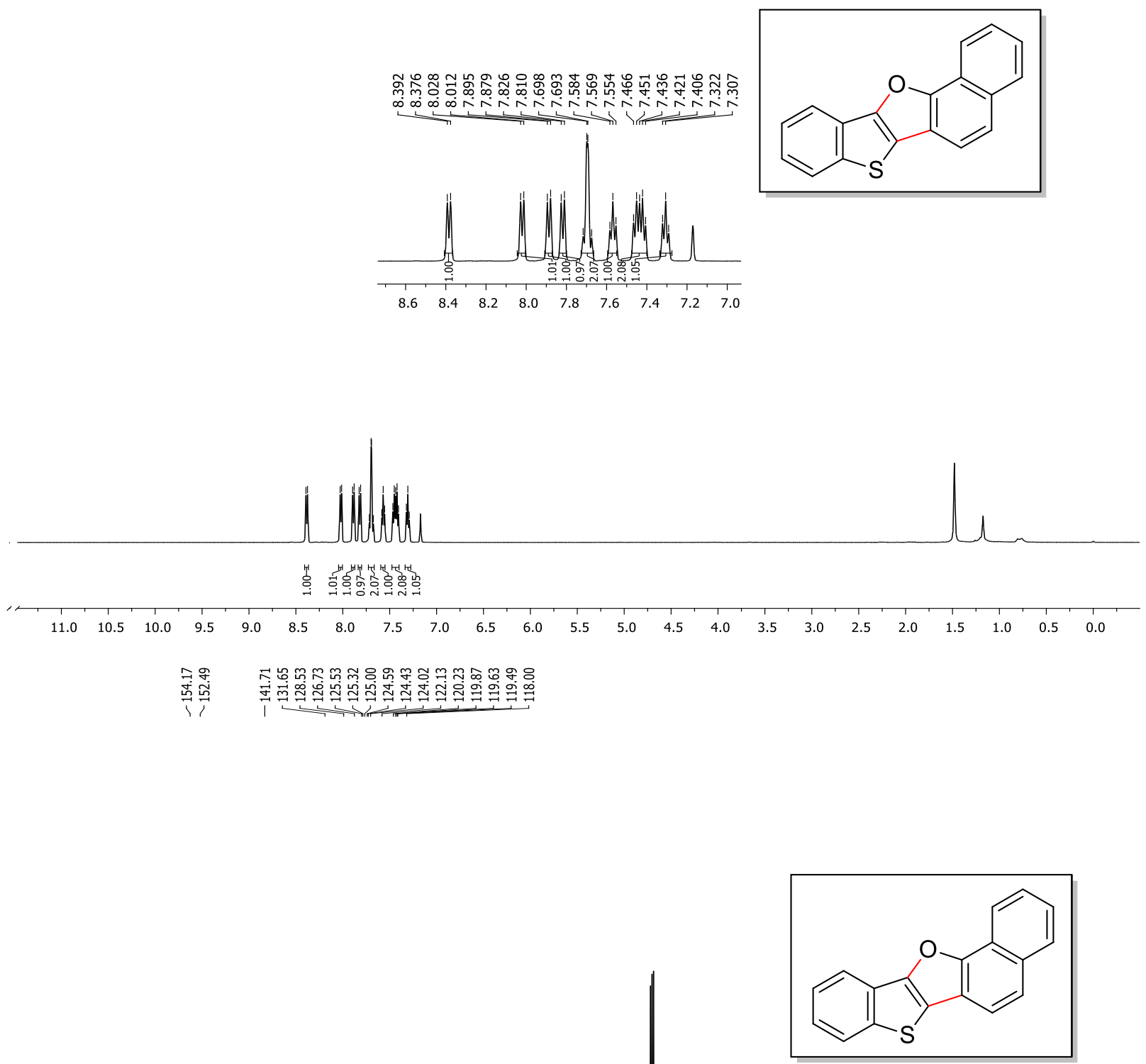

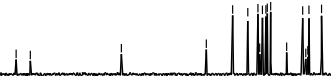

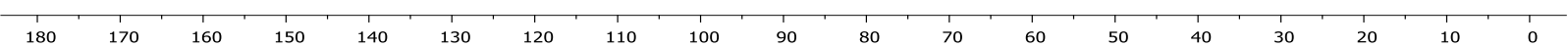




\section{COSY Spectra of $\mathbf{3 y}$}
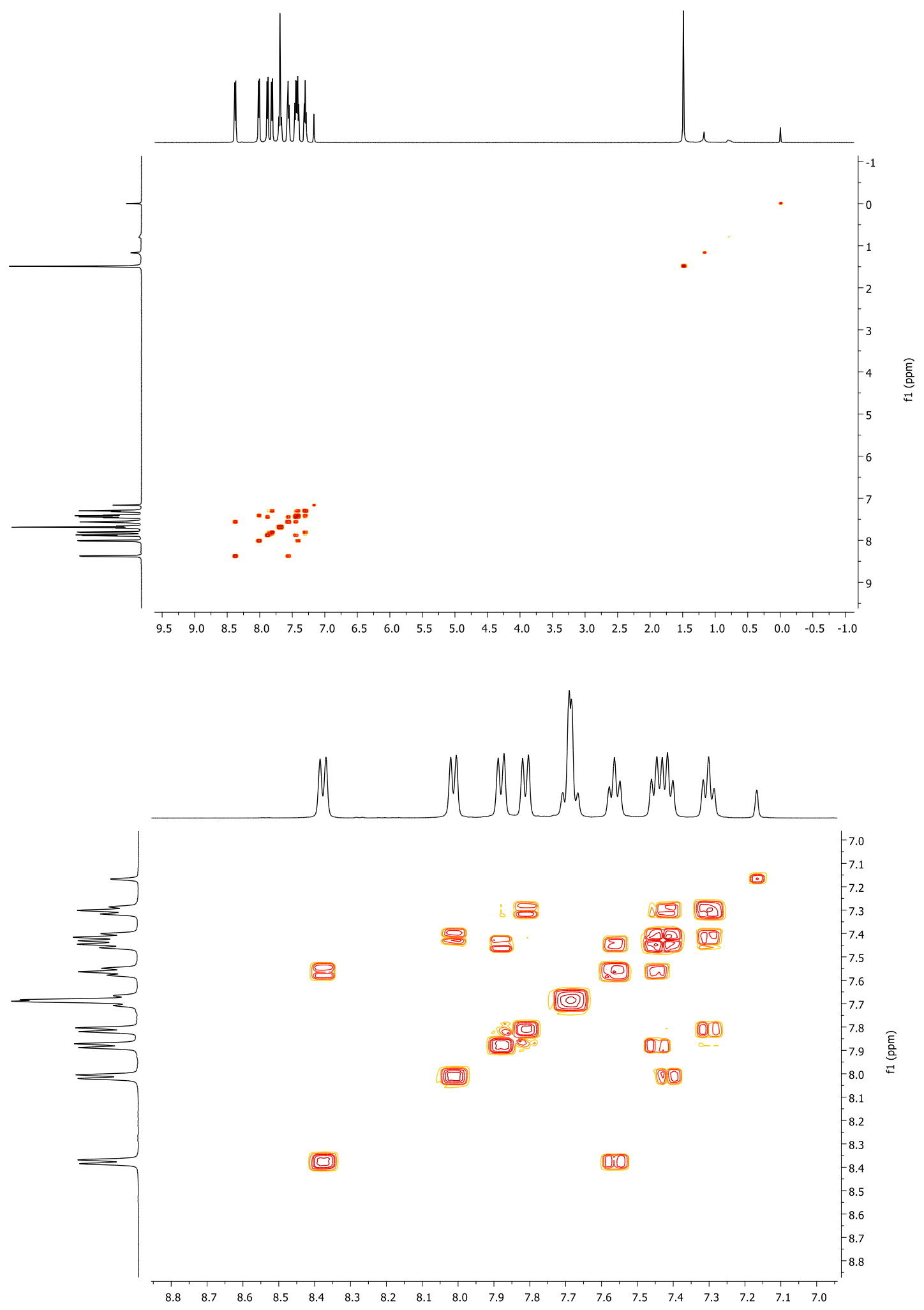


\section{HMQC Spectra of 3y}
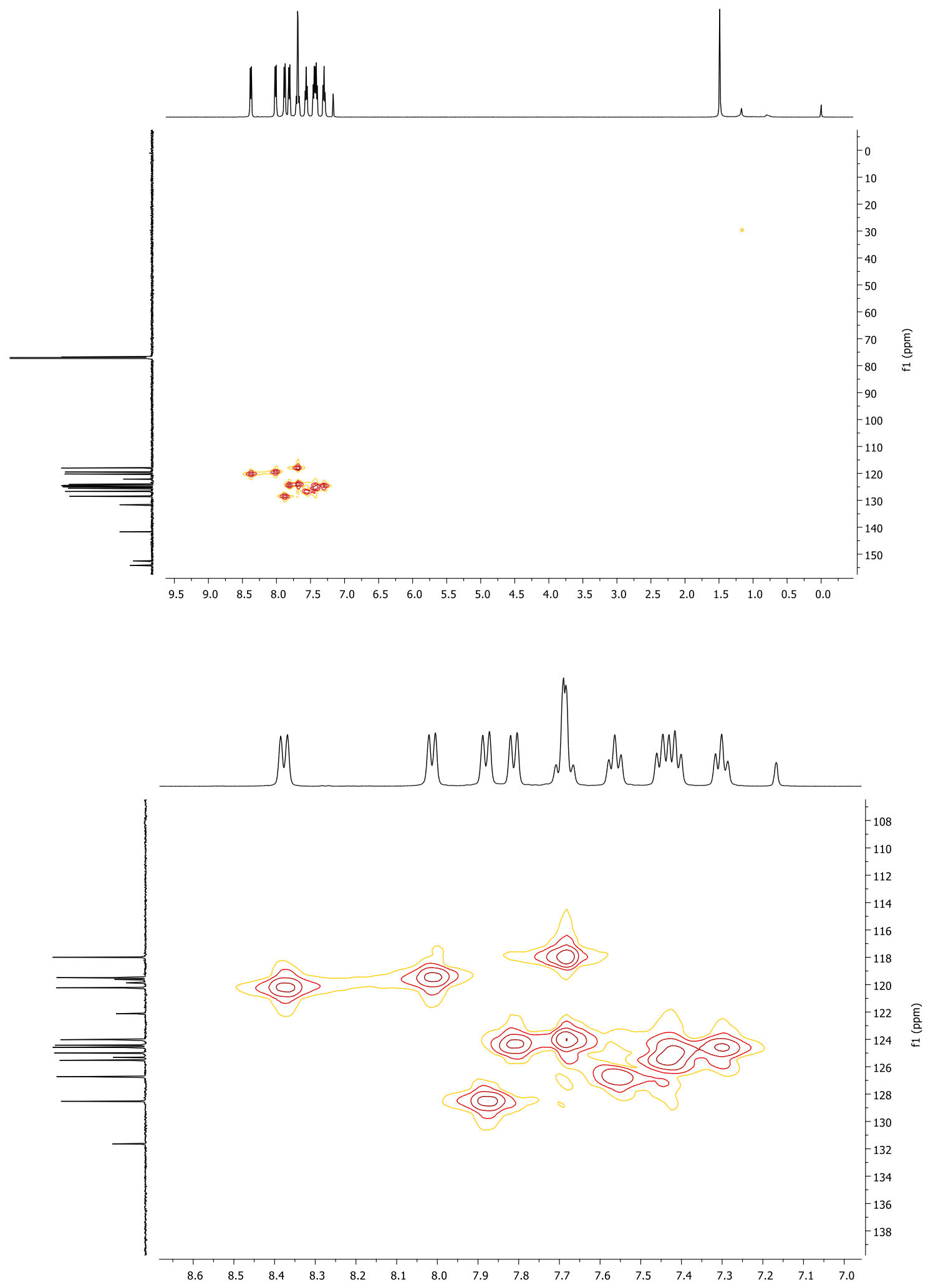
${ }^{1} \mathrm{H}$ NMR $\left(500 \mathrm{MHz}, \mathrm{CDCl}_{3}\right) \&{ }^{13} \mathrm{C}\left\{{ }^{1} \mathrm{H}\right\}\left(125 \mathrm{MHz}, \mathrm{CDCl}_{3}\right)$ Spectra of $\mathbf{3 z}$

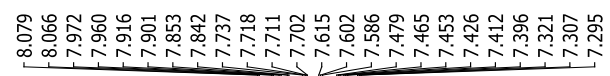
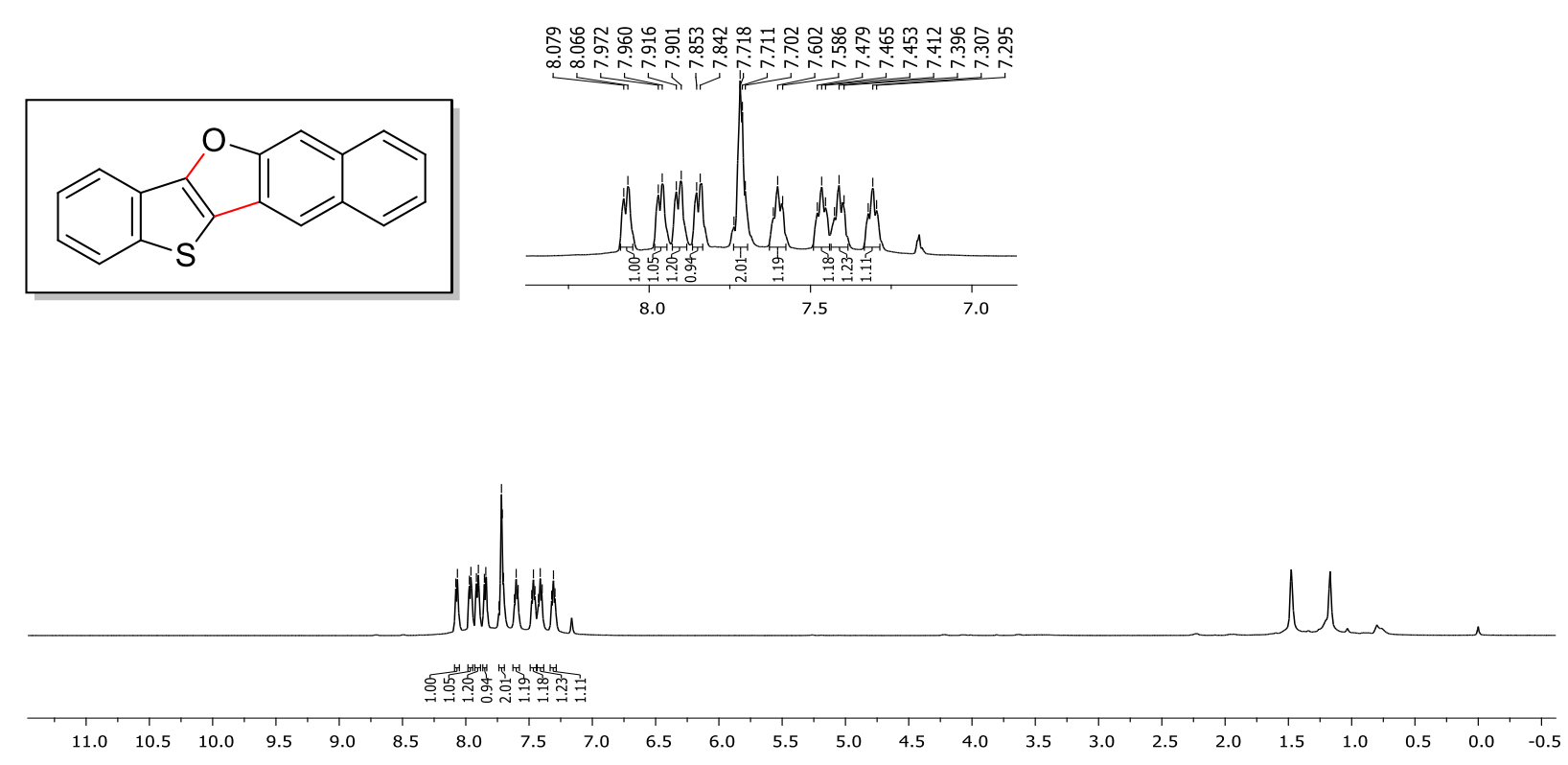

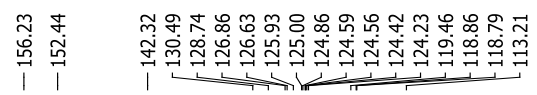

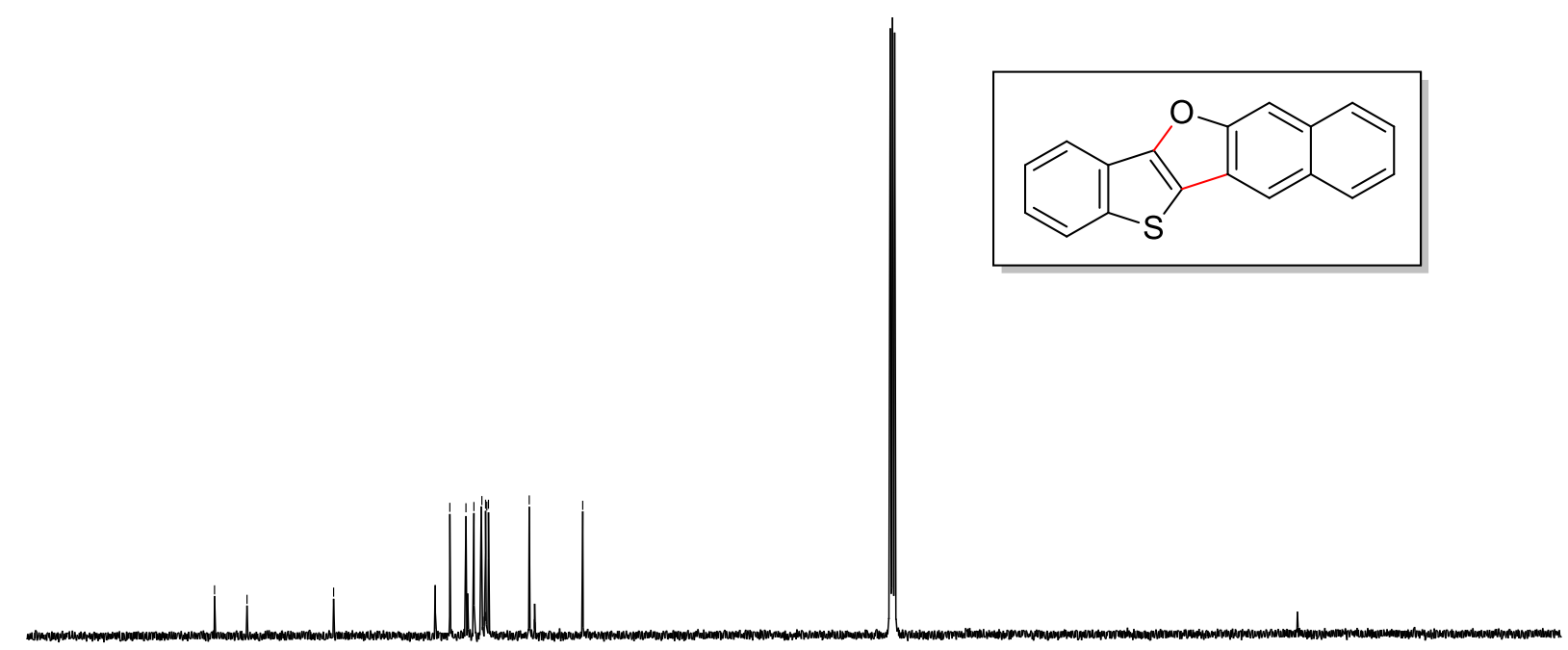




\section{COSY Spectra of $\mathbf{3 z}$}
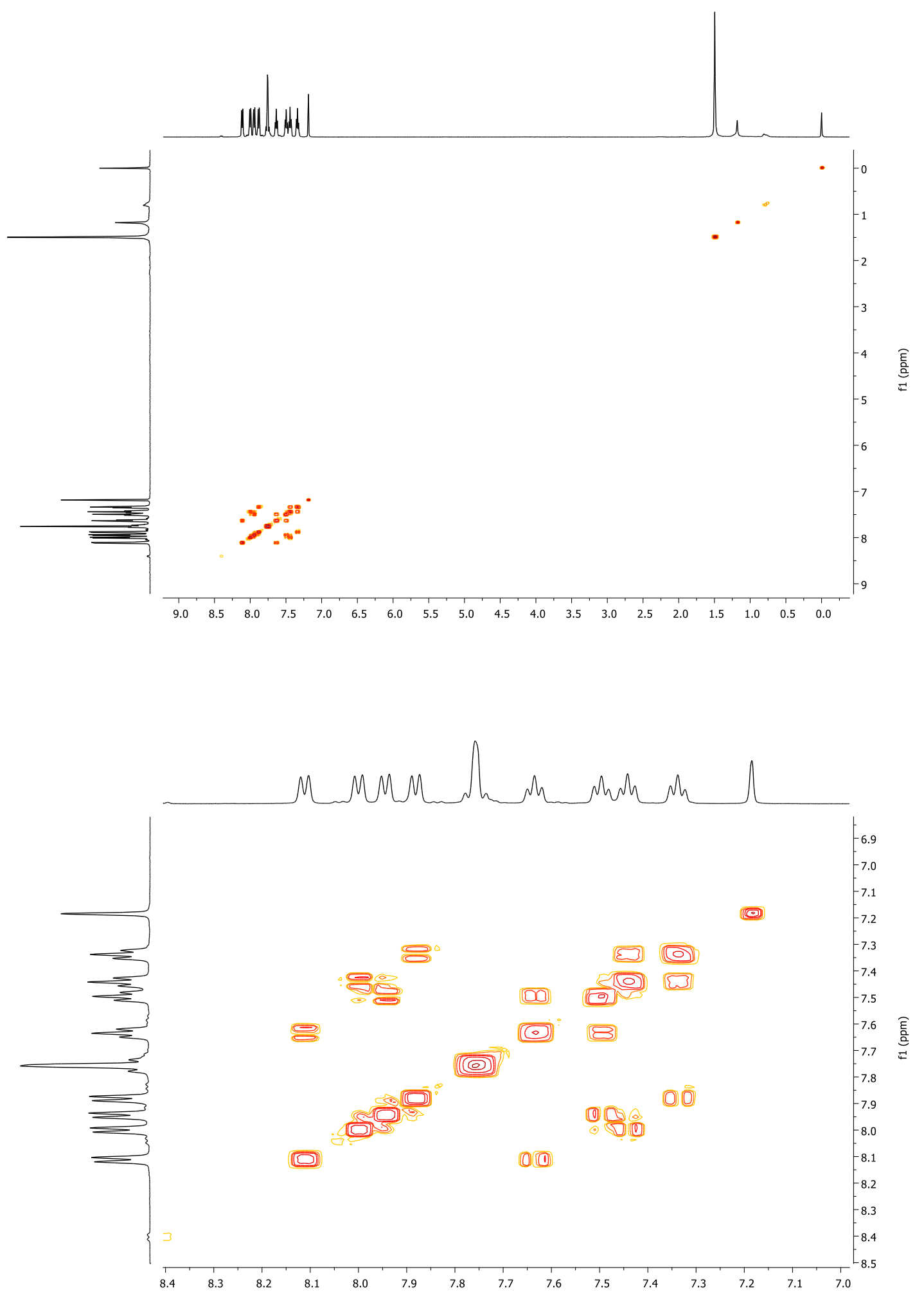


\section{HMQC Spectra of $\mathbf{3 z}$}
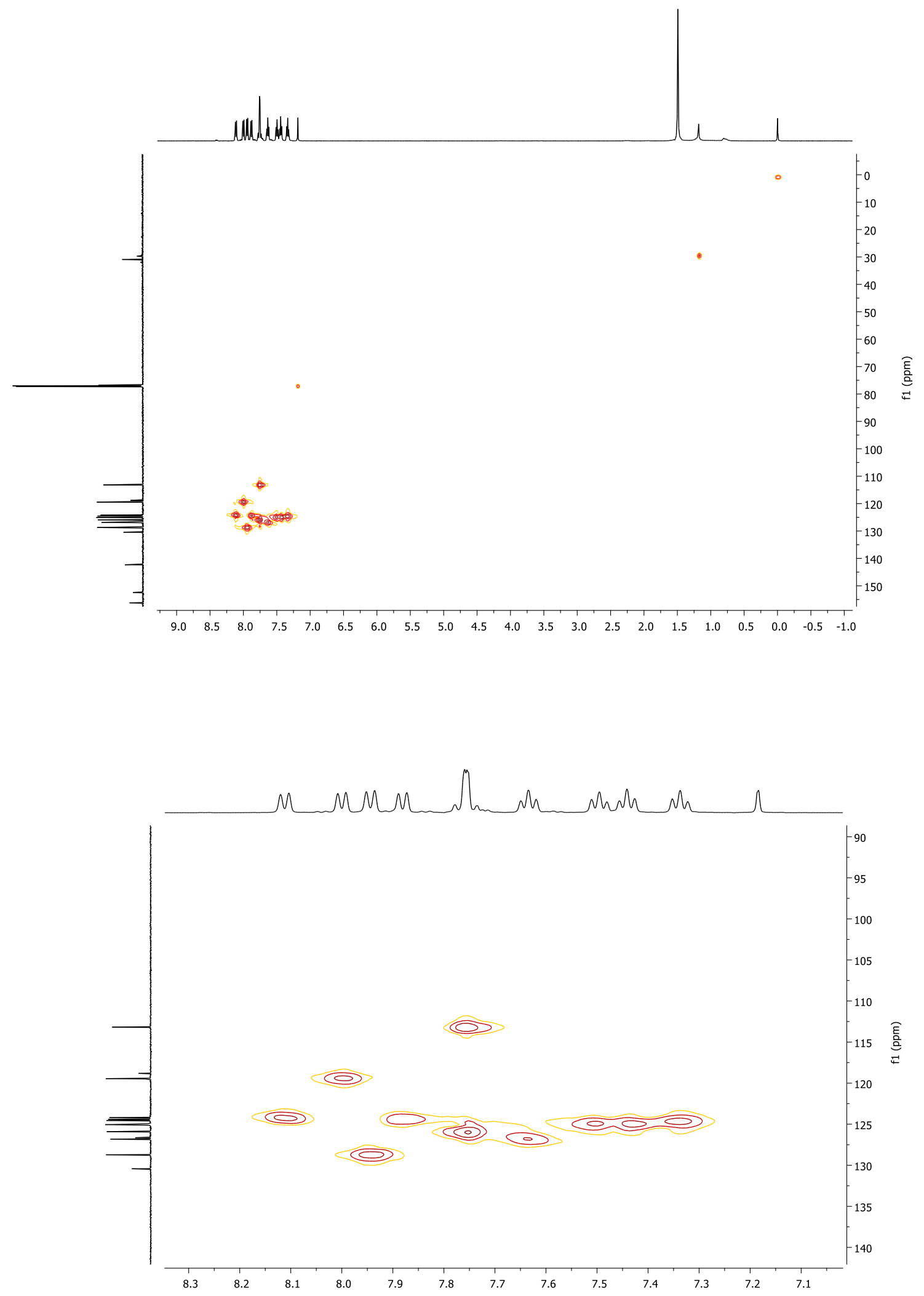
${ }^{1} \mathrm{H}$ NMR $\left(500 \mathrm{MHz}, \mathrm{CDCl}_{3}\right) \&{ }^{13} \mathrm{C}\left\{{ }^{1} \mathrm{H}\right\}\left(125 \mathrm{MHz}, \mathrm{CDCl}_{3}\right)$ Spectra of 3aa

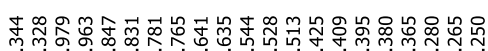

Whintrint
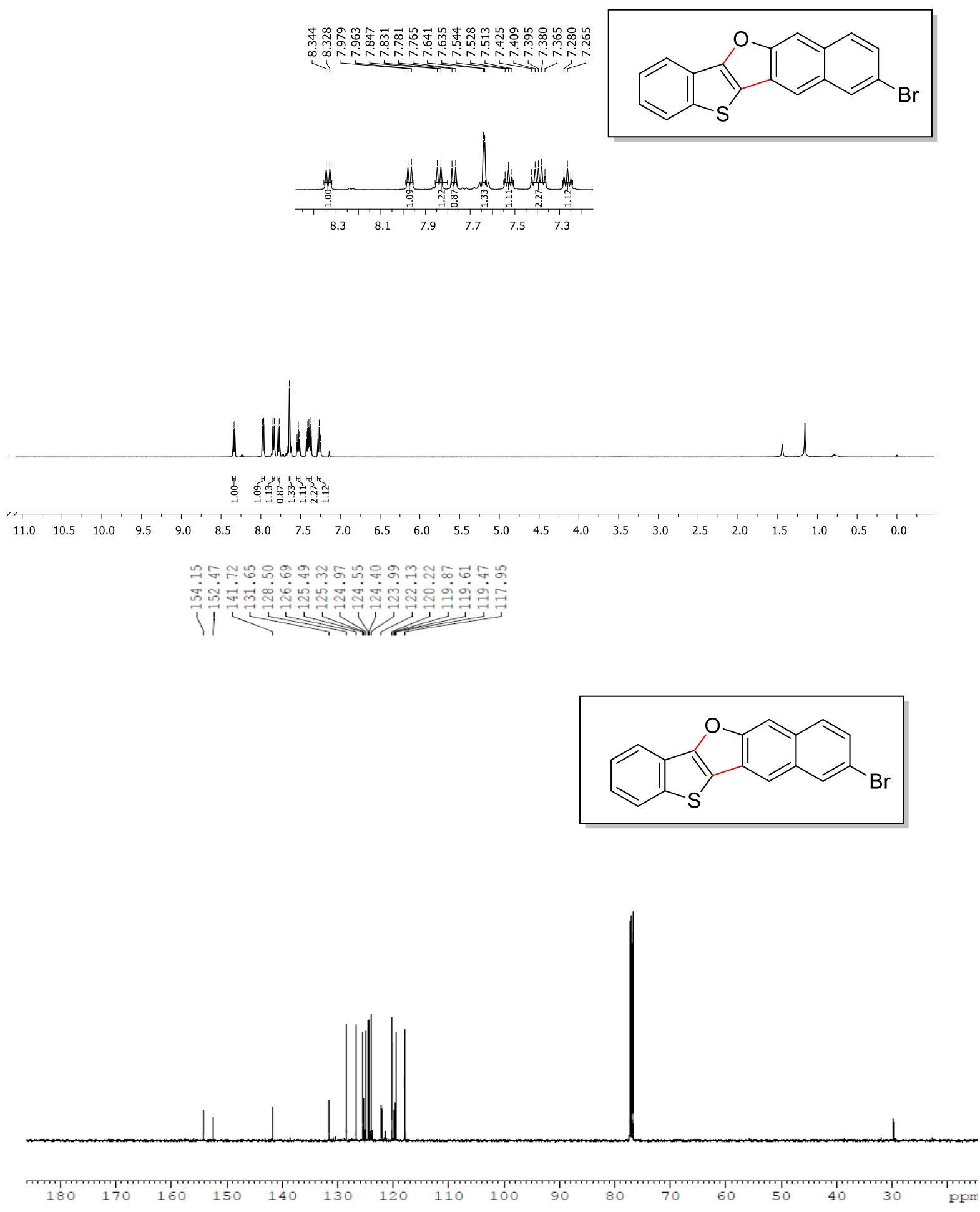
${ }^{1} \mathrm{H}$ NMR $\left(500 \mathrm{MHz}, \mathrm{CDCl}_{3}\right) \&{ }^{13} \mathrm{C}\left\{{ }^{1} \mathrm{H}\right\}\left(125 \mathrm{MHz}, \mathrm{CDCl}_{3}\right)$ Spectra of 3ab
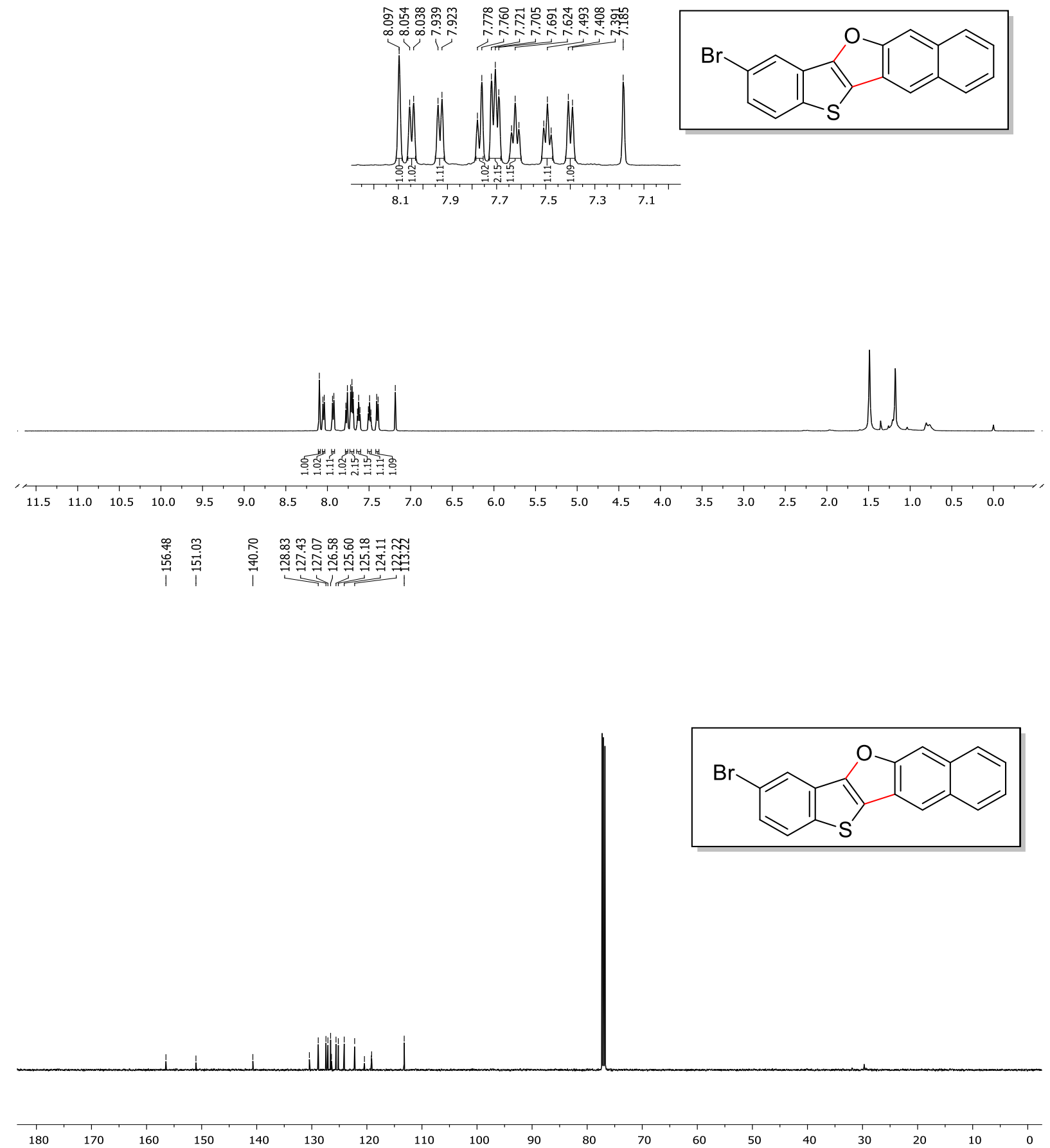
${ }^{1} \mathrm{H}$ NMR $\left(500 \mathrm{MHz}, \mathrm{CDCl}_{3}\right) \&{ }^{13} \mathrm{C}\left\{{ }^{1} \mathrm{H}\right\}\left(125 \mathrm{MHz}, \mathrm{CDCl}_{3}\right)$ Spectra of $\mathbf{5}$

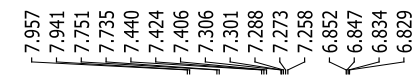

$\underset{\infty}{\stackrel{\varpi}{i ~}}$
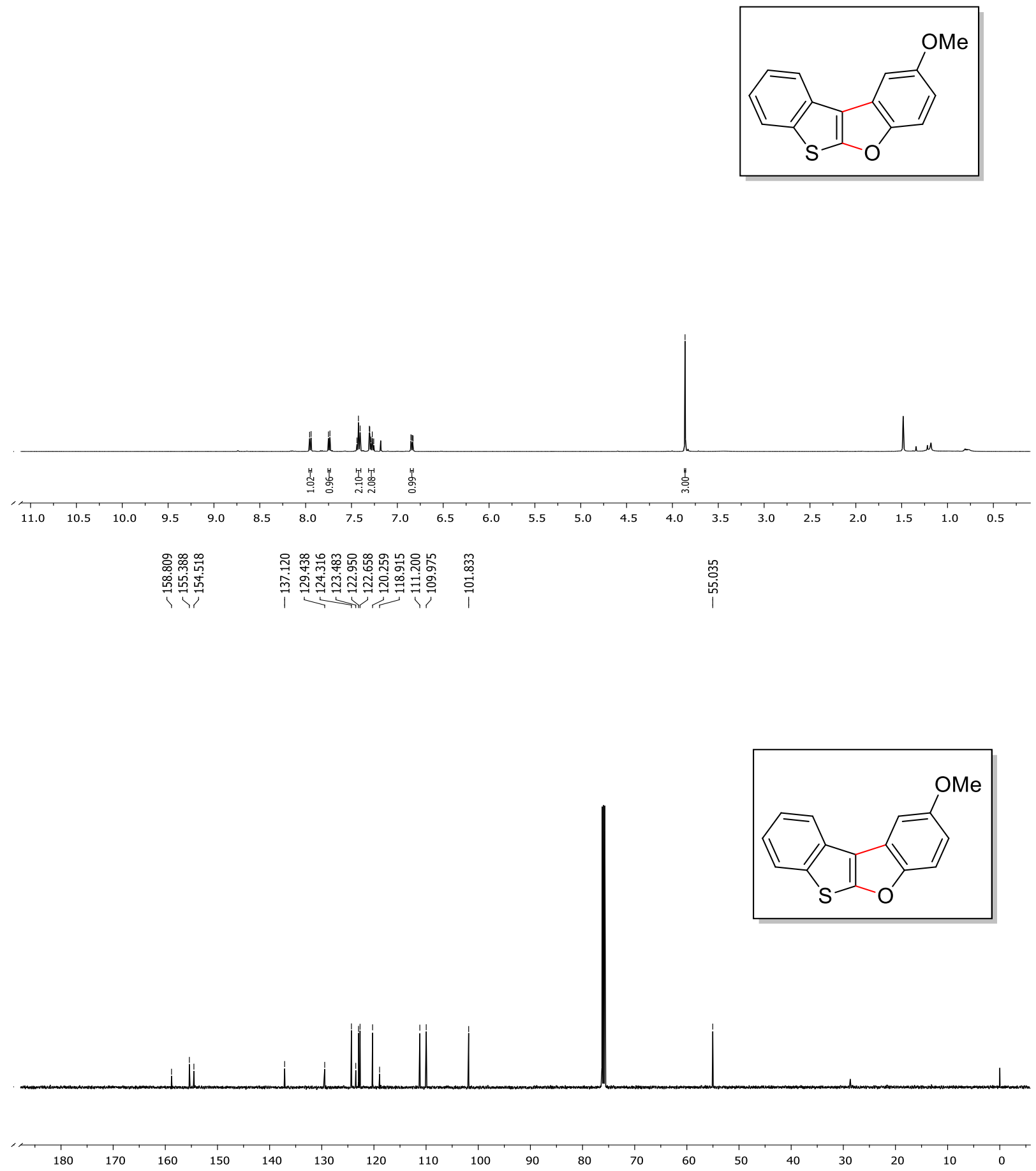
${ }^{1} \mathrm{H}$ NMR $\left(500 \mathrm{MHz}, \mathrm{CDCl}_{3}\right) \&{ }^{13} \mathrm{C}\left\{{ }^{1} \mathrm{H}\right\}\left(125 \mathrm{MHz}, \mathrm{CDCl}_{3}\right)$ Spectra of 7
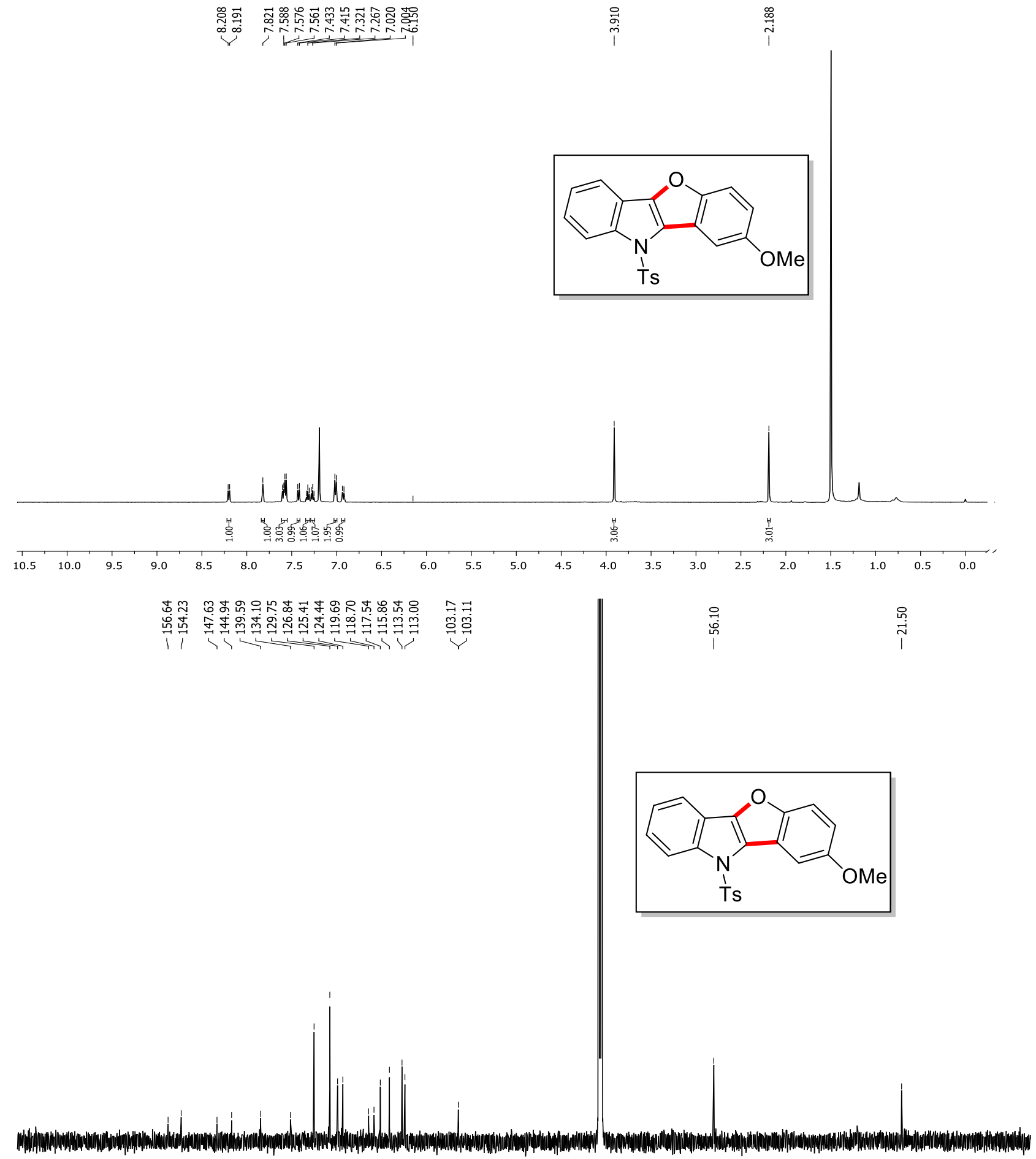
${ }^{1} \mathrm{H}$ NMR $\left(500 \mathrm{MHz}, \mathrm{DMSO}-\mathrm{d}_{6}\right) \&{ }^{13} \mathrm{C}\left\{{ }^{1} \mathrm{H}\right\}\left(125 \mathrm{MHz}, \mathrm{DMSO}-\mathrm{d}_{6}\right)$ Spectra of 8

1
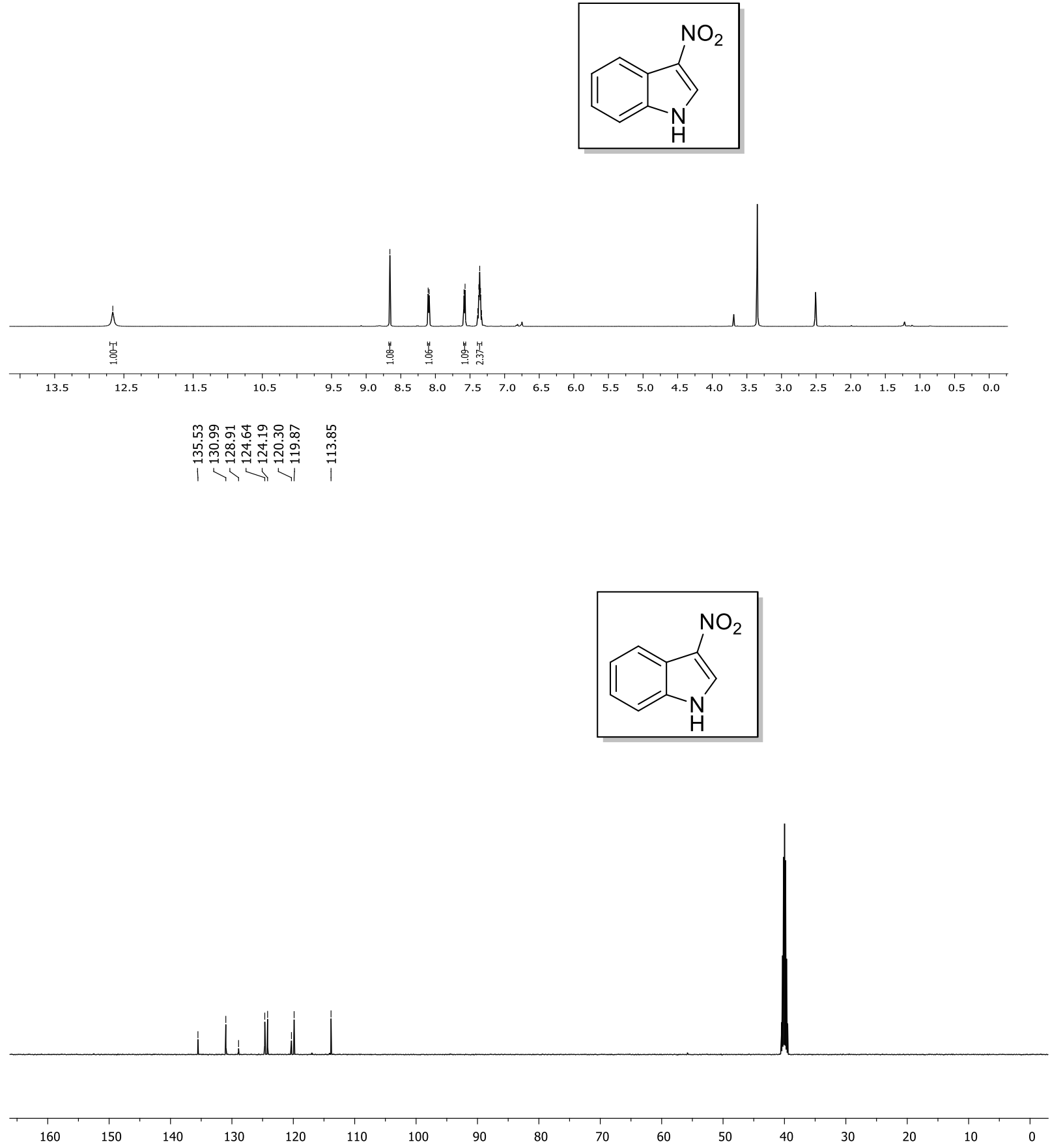
${ }^{1} \mathrm{H}$ NMR $\left(500 \mathrm{MHz}, \mathrm{CDCl}_{3}\right) \&{ }^{13} \mathrm{C}\left\{{ }^{1} \mathrm{H}\right\}\left(125 \mathrm{MHz}, \mathrm{CDCl}_{3}\right)$ Spectra of 9

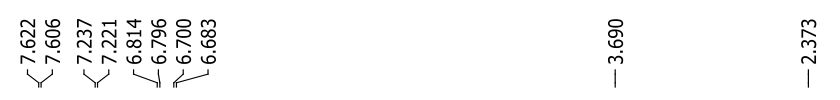
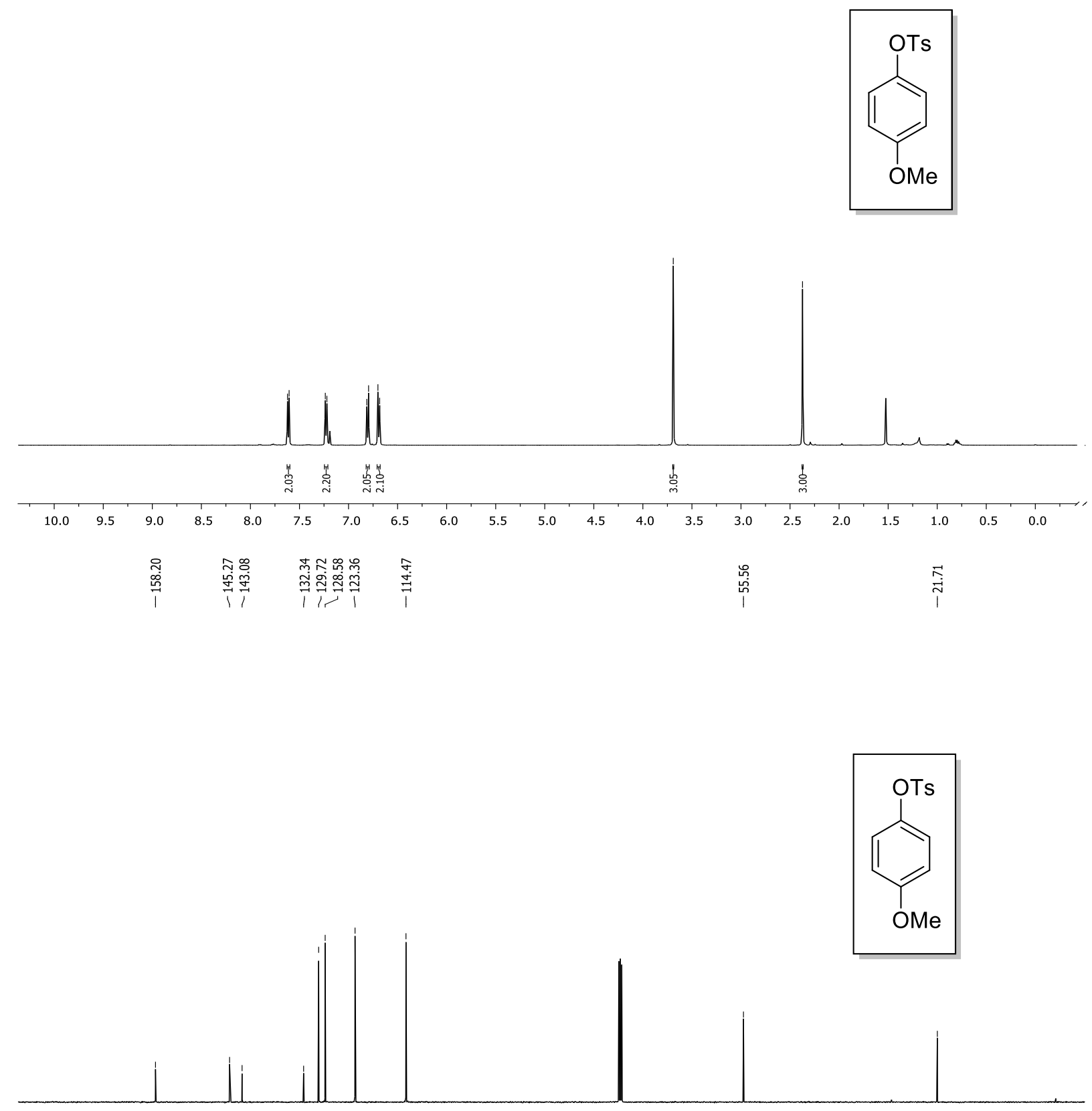


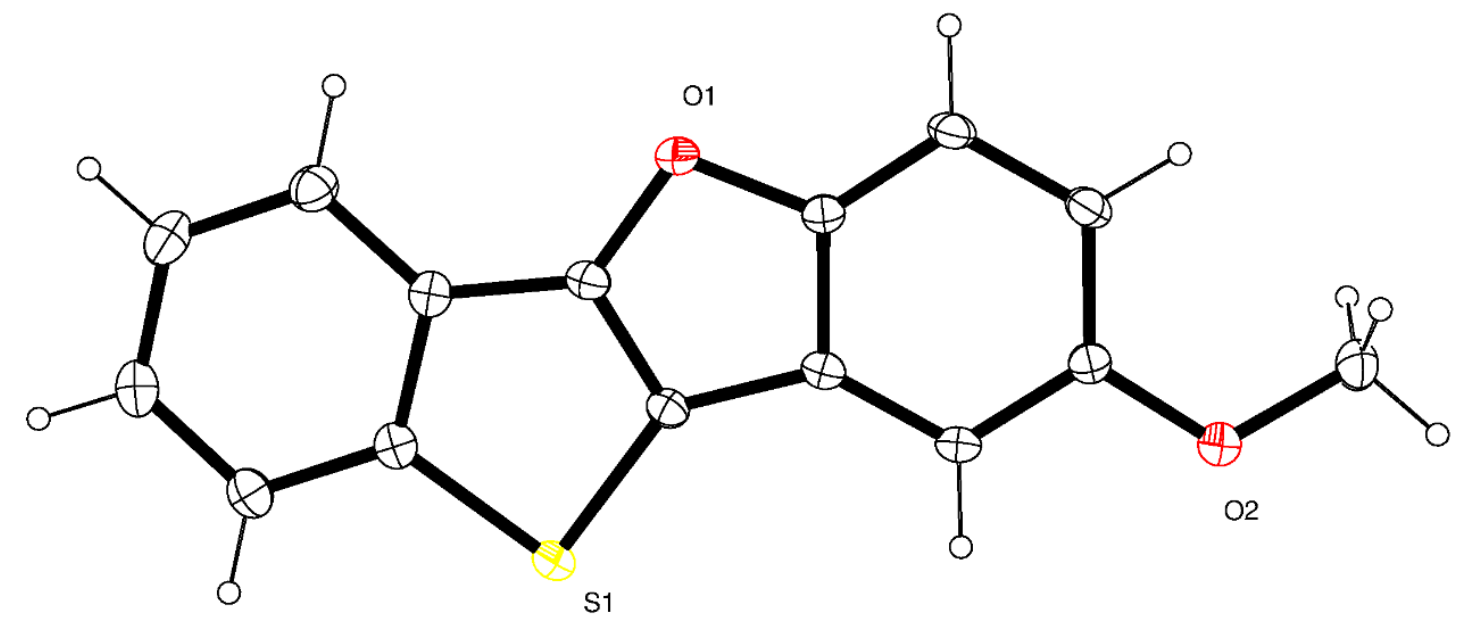

Single crystal X-ray of 3a (CCDC: $2055375,30 \%$ ellipsoid contour probability level)

Table 1. Crystal data and structure refinement for akl133.

Identification code

Empirical formula

Formula weight

Temperature

Wavelength

Crystal system

Space group

Unit cell dimensions

Volume

$\mathrm{Z}$

Density (calculated)

Absorption coefficient

$\mathrm{F}(000)$

Crystal size

Theta range for data collection

Index ranges

Reflections collected shelx

C15 H10 O2 S

254.29

296(2) K

$0.71073 \AA$

Orthorhombic

P 212121

$a=5.1327(4) \AA$ $\alpha=90^{\circ}$.

$\mathrm{b}=14.7662(13) \AA$

$\beta=90^{\circ}$.

$c=15.2136(14) \AA$ $\gamma=90^{\circ}$.
4

$1.465 \mathrm{Mg} / \mathrm{m}^{3}$

$0.269 \mathrm{~mm}^{-1}$

528

$0.170 \times 0.038 \times 0.035 \mathrm{~mm}^{3}$

2.678 to $24.995^{\circ}$.

$-6<=\mathrm{h}<=6,-17<=\mathrm{k}<=16,-18<=\mathrm{l}<=18$

6539 
Independent reflections

Completeness to theta $=24.995^{\circ}$

Absorption correction

Max. and min. transmission

Refinement method

Data / restraints / parameters

Goodness-of-fit on $\mathrm{F}^{2}$

Final R indices [I $>2 \operatorname{sigma}(\mathrm{I})]$

$\mathrm{R}$ indices (all data)

Absolute structure parameter

Extinction coefficient

Largest diff. peak and hole
$2023[\mathrm{R}($ int $)=0.0370]$

$99.9 \%$

Semi-empirical from equivalents

0.991 and 0.956

Full-matrix least-squares on $\mathrm{F}^{2}$

2023 / 0 / 165

1.054

$\mathrm{R} 1=0.0312, \mathrm{wR} 2=0.0692$

$\mathrm{R} 1=0.0373, \mathrm{wR} 2=0.0727$

$-0.05(5)$

$0.021(3)$

0.134 and -0.147 e. $\AA^{-3}$ 